DANMARKS GEOLOGISKE UNDERS $\varnothing$ GELSE · DGU SERIE B · NR. 13 MILJØMINISTERIET · Geological Survey of Denmark · DGU SERIES B · No. 13

Geochemistry of the Upper Permian Ca-2 Deposits of the Løgumkloster-1 Well, South Denmark

BY

NIELS STENTOFT, PETER FRYKMAN, POVL VALDEMAR ANDERSEN AND KAARE LUND RASMUSSEN

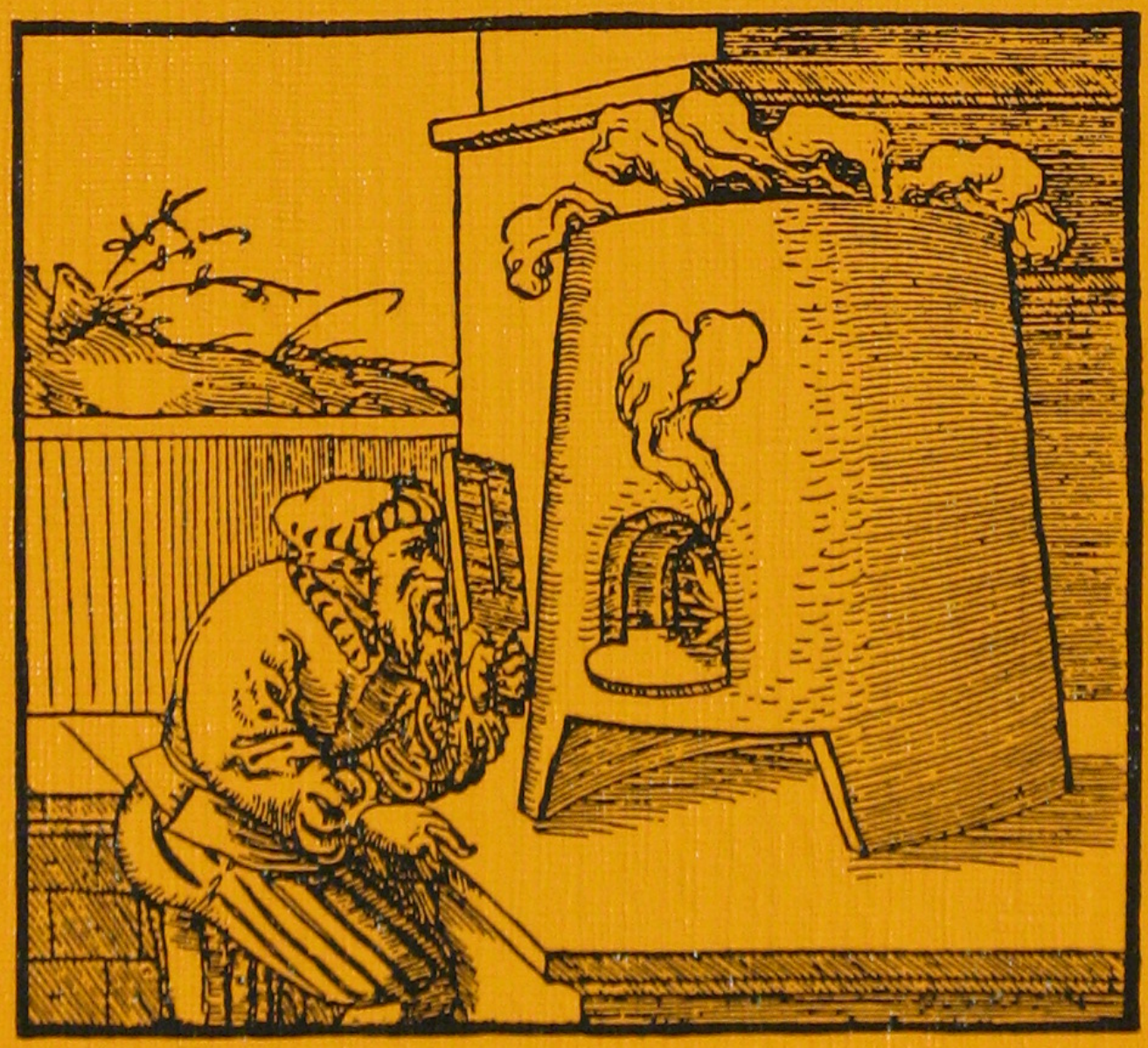

Danmarks Geologisk Undersøgelse · København 1990 


\section{Geochemistry of the Upper Permian Ca-2 Deposits of the Løgumkloster-1 Well, South Denmark}

BY

NIELS STENTOFT, PETER FRYKMAN, POVL VALDEMAR ANDERSEN AND KAARE LUND RASMUSSEN 
Keywords:

Geochemistry, Zechstein, Denmark, Dolomite, Anhydrite, Evaporite.

\section{Vignette:}

“... the assayer ... should close the doors of the room in which the assay furnace stands, lest anyone coming at an inopportune moment might disturb his thoughts when they are intent on the work."

(Agricola, G. (1556): De re metallica)

DGU $\cdot$ serie B $\cdot$ nr. 13

ISBN 87-88640-51-5

ISSN 0901-0289

Oplag: 1000

Tryk: AiO Tryk as, Odense

Tegning: Anna-Beth Andersen m.fl.

Foto: Svend Meldgaard Christiansen

Dato: $15-10-1990$

Niels Stentoft, Peter Frykman, and Povl Valdemar

Andersen, Geological Survey of Denmark,

Thoravej 8, DK-2400 Copenhagen NV, Denmark.

Kaare Lund Rasmussen, Carbon-14 Dating

Laboratory, Danish National Museum and

Geological Survey of Denmark, Ny Vestergade 11,

DK-1471 Copenhagen K, Denmark.

Redaktion: Leif Banke Rasmussen

(C) Danmarks Geologiske Undersøgelse,

Thoravej 8, DK-2400 København NV.

I kommission hos: Geografforlaget Aps.

Ekspedition: Fruerhøjvej 43, 5464 Brenderup

Telefon 64441683 


\section{Contents}

\begin{tabular}{|c|c|c|}
\hline Abstract & 5 & Discussion .. \\
\hline Introduction. . . . . . . . . . . & 7 & Anhydrite....... \\
\hline Sample preparation and analytical pro- & & Dolomite ....... \\
\hline cedures $\ldots \ldots \ldots \ldots \ldots \ldots \ldots$ & 12 & The salt phases $\ldots \ldots \ldots \ldots \ldots \ldots$ \\
\hline Sampling. . . . . . . . . . . & 12 & The EDTA-insoluble phases..... \\
\hline Thin and thick sections $\ldots \ldots \ldots$ & 12 & Rare Earth Elements, Sc, and Th.. \\
\hline Instrumental Neutron Activation & & Iron $\ldots \ldots \ldots \ldots \ldots \ldots \ldots$ \\
\hline Analysis (INAA) . . . . . . . . . & 12 & Antimony, As, and Se..... \\
\hline Loss on ignition and wet chemical & & Chromium and $\mathrm{U} \ldots \ldots \ldots$ \\
\hline analysis $\ldots \ldots \ldots \ldots \ldots \ldots$ & 12 & Hafnium, Ta, and Th.......... \\
\hline X-ray diffraction $\ldots \ldots \ldots \ldots \ldots$ & 13 & The highly siderophile elements ... \\
\hline Magnetic measurements . . . . . . . & 13 & The drilling mud $\ldots \ldots \ldots \ldots \ldots$ \\
\hline Results $\ldots \ldots \ldots \ldots \ldots \ldots \ldots$ & 18 & Conclusions $\ldots \ldots \ldots \ldots \ldots \ldots \ldots$ \\
\hline & & Acknowledgements ....... \\
\hline & & References $\ldots \ldots \ldots \ldots \ldots \ldots \ldots$ \\
\hline
\end{tabular}




\section{Abstract}

Rock samples of the Upper Permian Ca-2 unit of the Løgumkloster-1 well have been subjected to chemical analysis. The $\mathrm{Ca}-2$ interval was undoubtedly originally an aragonite which have undergone several episodes of diagenesis including a dolomitization and a heavy anhydrite mineralization. Two original facies types are recognized, an oolitic shoal facies, and a lagoonal carbonate facies.

The samples have been characterized by neutron activation, atomic absorption, Xray diffraction, palaeomagnetism, optical and electron microscopical examination. Samples of the drilling mud has been analyzed as well, and it is found that contamination of the samples included in the present study from the drilling mud is unlikely to have happened. Imprints of both the original facies and the diagenetic events are found in the present work. The $\mathrm{Sr}$ and $\mathrm{Ba}$ abundances reflect the original facies-bound distribution, which is also reflected in the normalized REE distribution patterns. A low-Sr anhydrite component is clearly distinguishable in several parameter cross plots. Iron, Sc, REE and several other elements are found to be situated in the EDTA-insoluble residue, which has been identified primarily as a muscovite or clay mineral phase. Pyrite is present, although of small relative volume it is thought to be responsible for the occurrence of $\mathrm{As}, \mathrm{Sb}$, and possibly Se. A small fraction of the $\mathrm{Cr}$, $\mathrm{As}$, and $\mathrm{Sb}$ may originate from the heavy part of the crude oil, the introduction of which was the last process of alteration the rock experienced. A geomagnetically reversed period is encountered in the middle of the Ca-2 interval, and is tentatively identified as one of the Tatarian reversed events. 


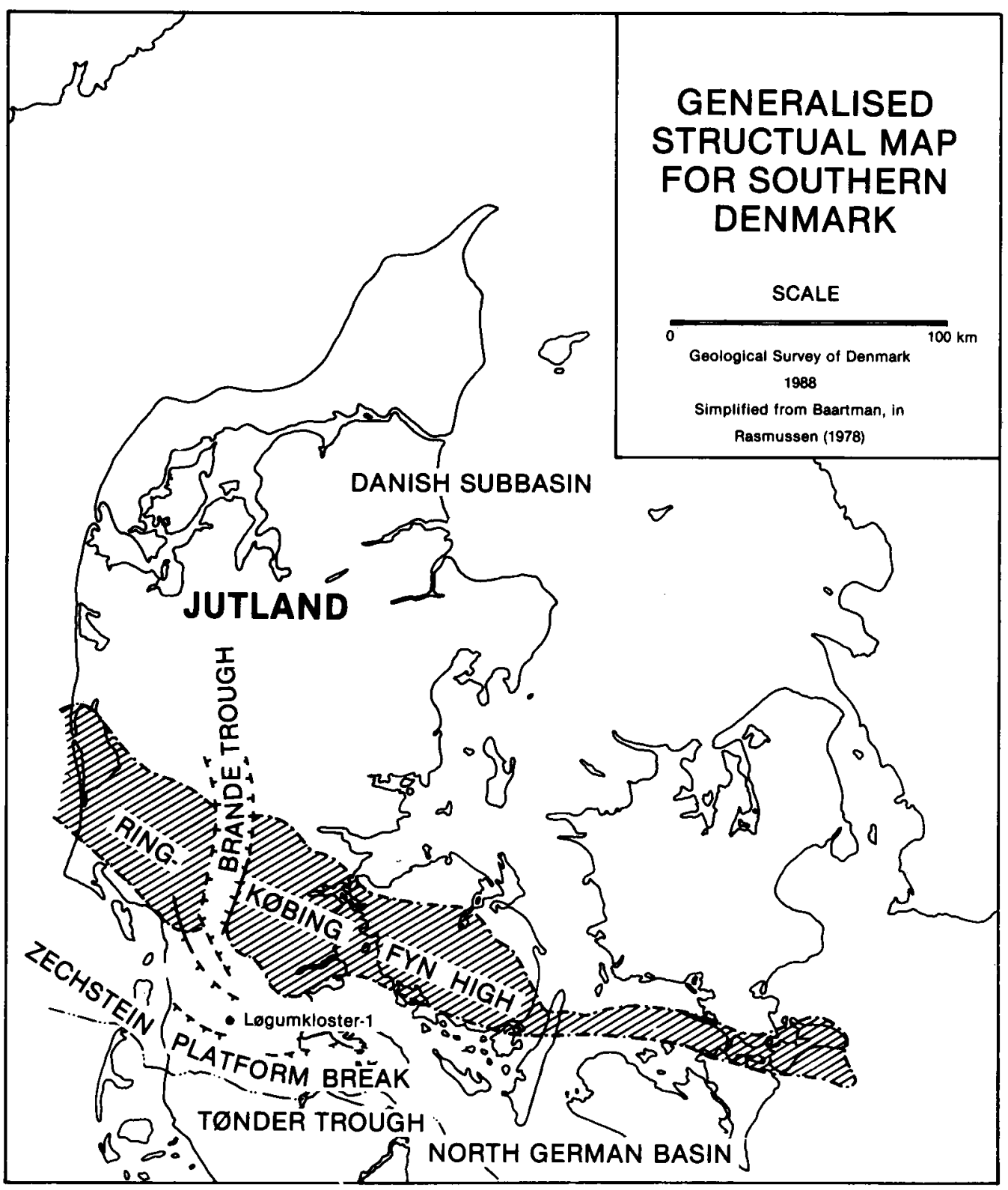

Fig. 1. Location of the Løgumkloster-1 well in southern Jylland, Denmark. 


\section{Introduction}

The Løgumkloster-1 well is located in Southern Jylland, Denmark (Figure 1). During Upper Permian this area was situated on the southern slope of the Ringkøbing-Fyn High, which formed the northern margin of the North German Zechstein Basin (Figure 1; Baartman in Rasmussen, 1978).

The Zechstein Group in the Løgumkloster- 1 well is $531 \mathrm{~m}$ thick. It includes at least three sedimentary cycles: Z1, Z2 and Z3 as shown on Figure 2 (Richter-Bernburg, 1955; Clark \& Tallbacka, 1980). The ca. 41 $\mathrm{m}$ thick Ca-2 carbonate unit (ca. $2424 \mathrm{~m}$ ca. $2465 \mathrm{~m} \mathrm{~b}$. KB) studied in this work, corresponds to the Stassfurt-Karbonat of Germany.

The Ca-2 carbonate of Løgumkloster- 1 is a light-colored dolomite, including varying amounts of anhydrite. The dolomite is primarily derived from sediments of coated grains, which descriptively can be divided into ooids and oncoids ("eggs" and "nodules") (Figure 3). According to the carbonate texture (Dunham, 1962) the sequence can be divided into three subunits with interval boundaries at ca. $2432 \mathrm{~m}$ and ca. $2443 \mathrm{~m}$ respectively (Figure 4 ). The carbonate of subunit II (ca. $2432 \mathrm{~m}$ - ca. $2443 \mathrm{~m}$ ) is an oolitic grain- to packstone. In subunits I (ca. $2443 \mathrm{~m}$ - ca. $2465 \mathrm{~m}$ ) and III (ca. $2424 \mathrm{~m}$ ca. $2432 \mathrm{~m})$ it is dominantly an oncolitic/ oolitic wacke- to grainstone, but locally oolitic/oncolitic mudstone occur. The rocks of both unit I and III are interbedded with several $\mathrm{mm}$ - to $\mathrm{cm}$-size layers of wavy laminated algal boundstone (Figure 4). In terms of general facies types the interval can be divided into an oolitic shoal facies (type A, including mainly subunit JI), and a lagoonal carbonate facies (type B, including mainly subunits I and III).

The definition of these facies, or sedimentary environments, is primarily based on the following criteria: morphology of the coated grains, presence of the wavy laminated boundstone which is undoubtedly algal

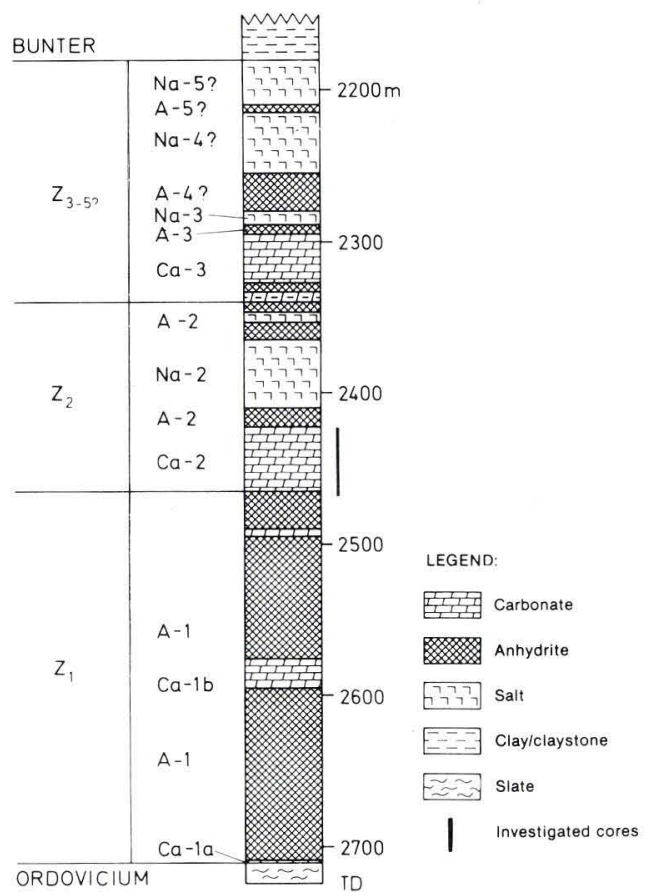

Fig. 2. Lithology and stratigraphy of the Zechstein sequence in the Løgumkloster-1 well, based on mud and wireline logs. The German nomenclature of Richter-Bernburg (1955) is used: $Z_{1}=$ Werra-Folge; $Z_{2}=$ Stassfurt-Folge; $Z_{3}=$ Leine-Folge; $Z_{4}=$ Aller-Folge; $Z_{5}=$ Ohre-Folge; $\mathrm{Ca}=$ Karbonat; $A=$ Sulfat; $\mathrm{Na}=$ Steinsalz; $C a-1=$ Werra-Karbonat; $A-1=$ Werra-Sulfat etc. 

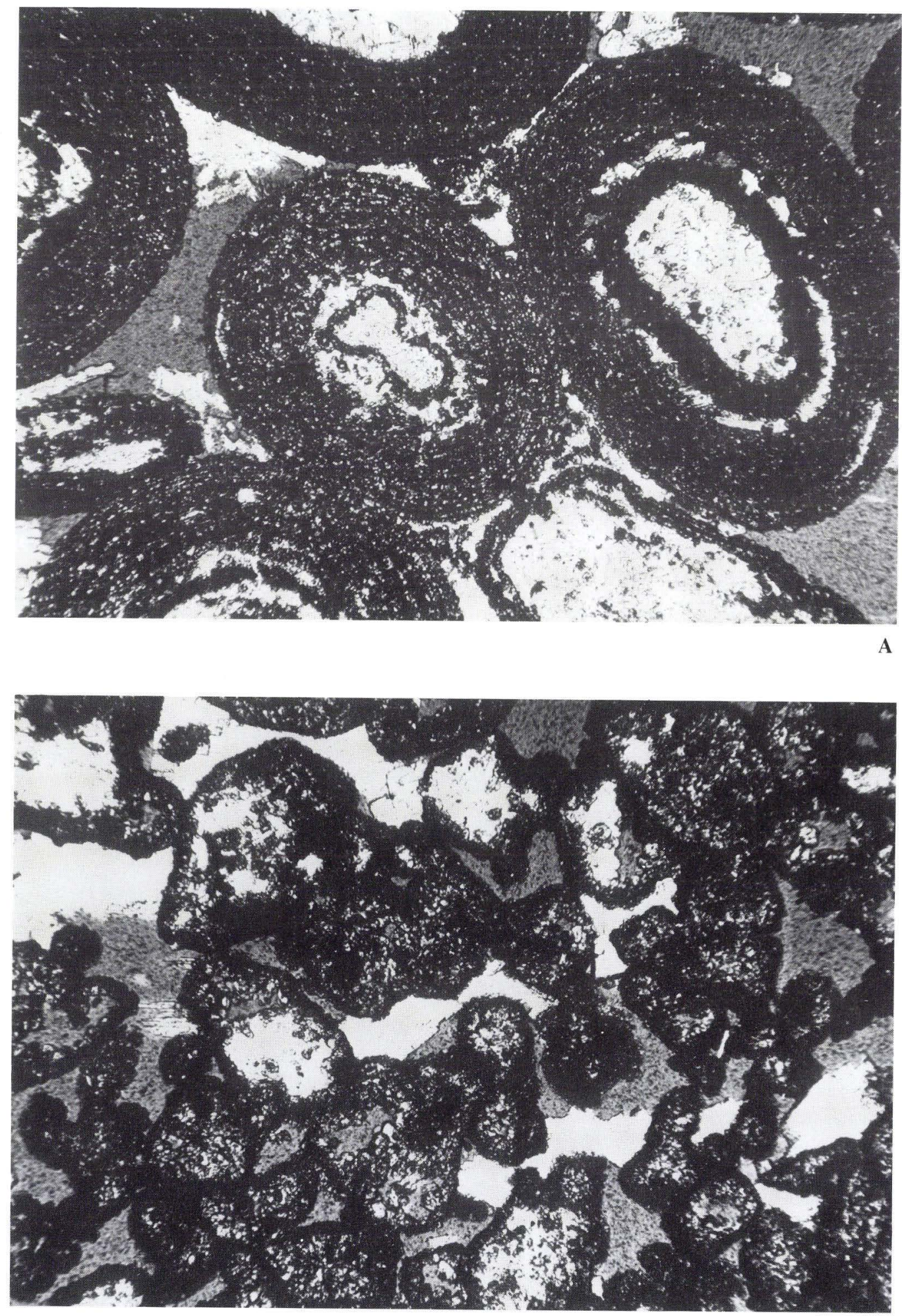


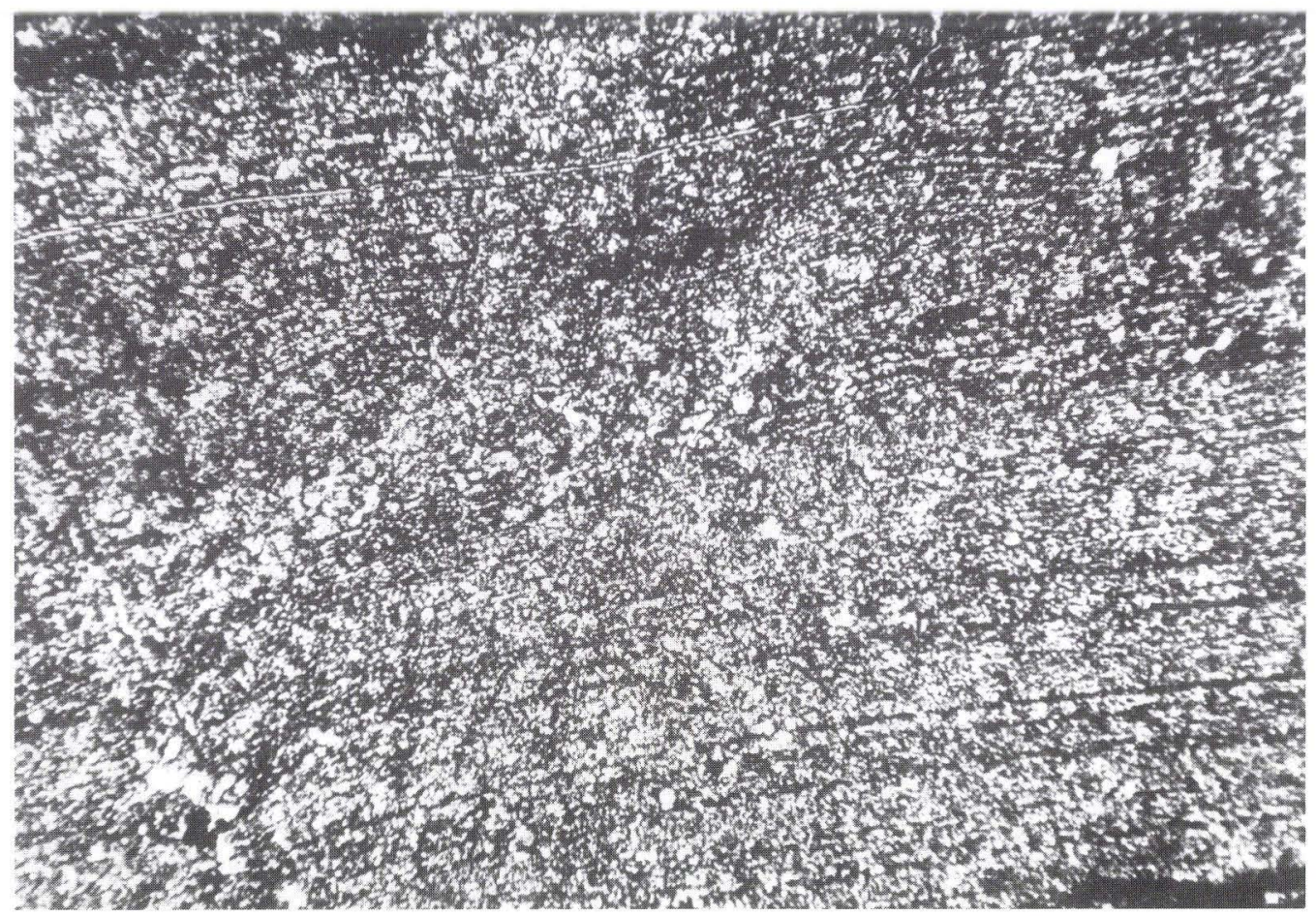

C

Fig. 3. Microphotographs of the most important Ca-2 carbonate types. A: Oolitic grainstone of the oolitic shoal facies. The concentric, algal-like structures are well preserved. The hollow ooids are filled with anhydrite (light colored). Some anhydrite have also precipitated around the ooids. (Sample 1756; depth: $2441.40 \mathrm{mb}$ b. KB). B: Oncolite grain- to wackestone of the lagoonal carbonate facies. The oncoids themselves are mostly preserved as irregular shaped, hollow shells of aphanocrystalline to very finely crystalline dolomite. The rock is partly cemented/replaced by anhydrite. (Sample 1801; depth: $2424.03 \mathrm{mb}$. KB). C: Algal boundstone of the lagoonal carbonate facies. The photo shows a part of a ca. $6 \mathrm{~mm}$ thick, wavy stromatolitic structure that runs parallel to the beddingplane, and is surrounded by oncoids. (Sample 1818; depth: $2455.82 \mathrm{~m} \mathrm{~b}$. KB).

Width of view is $2.2 \mathrm{~mm}$. 


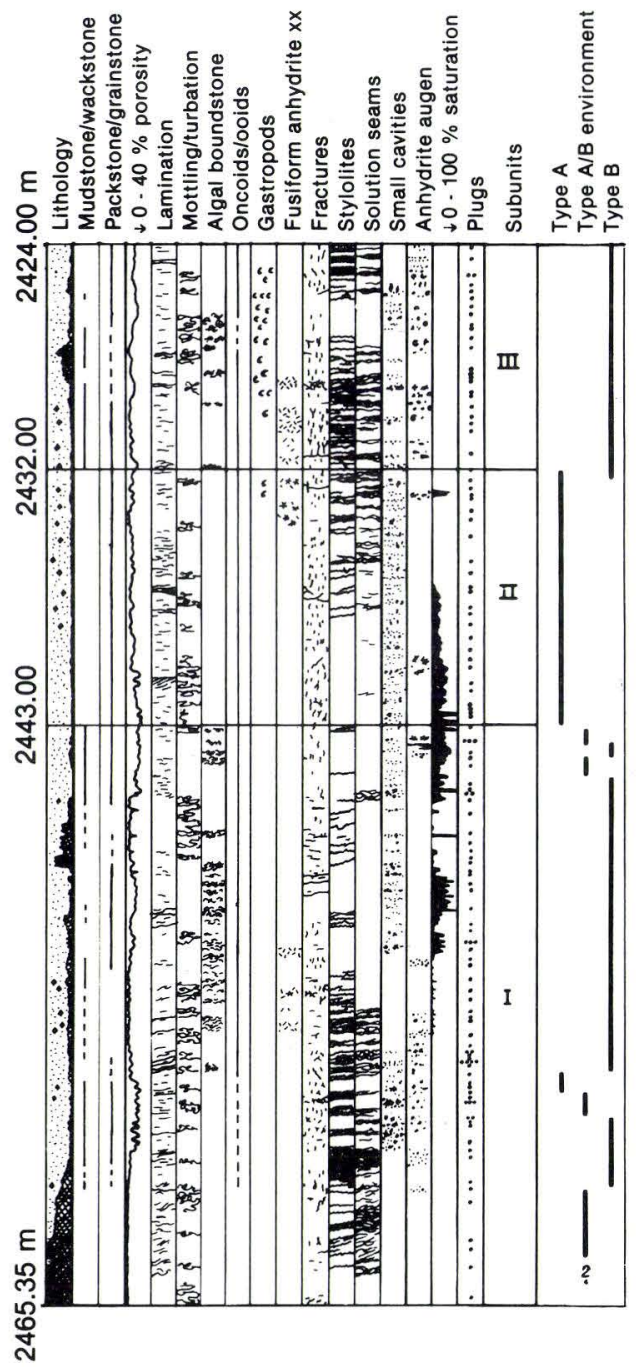

Fig. 4. Graphic core log of the Ca-2 unit in the Løgumkloster-1 well, based on macroscopic observations (from Stentoft, 1990).

in origin (cf. Figure 3), and sedimentary structures (Stentoft, 1990). The well rounded to perfectly spherical ooids of subunit II were formed in a high energy environment (Bathurst, 1976; Flügel, 1978) as confirmed by the presence of cross-laminations in the subunit. The ooids may have formed shoals or bar systems. In contrast to this environment, the rather irregular shaped, poorly sorted oncoids of subunits I and III were probably formed in a low energy environment, e.g. a lagoon, as they fulfill many of the criteria of "autochthonous ooids" (cf. Flügel, 1978, p. 125). The presence of stromatolitic algal structures just in the oncolitic intervals support the assumption that the wave action was rather slight. According to Logan et al. (1964, p. 77) similar algal structures may be found in intertidal mud-flat environments. For that reason the oncoidal carbonates of subunit I and III are suggested to have been deposited in a lagoonal environment in water depths ranging from shallow subtidal to upper intertidal.

Since deposition, the $\mathrm{Ca}-2$ sediments have experienced a complex diagenetic history as is outlined schematically in Figure 5 (Stentoft, 1990). The most important diagenetic events were as follows: (1) a phase of leaching with formation of inter- and intra-grain

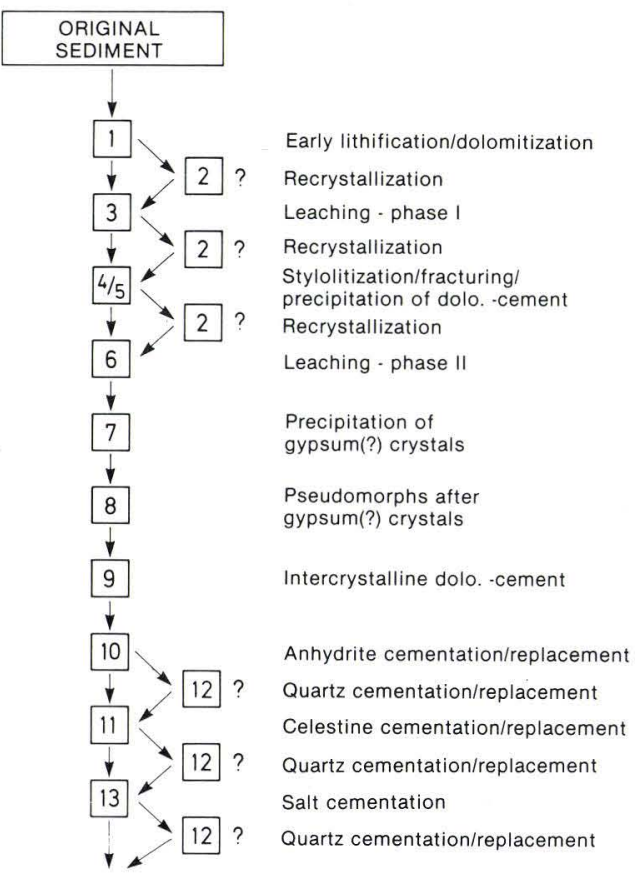

PRESENT ROCK

Fig. 5. Flow-chart for diagenetic evolution of the Ca-2 rock (from Stentoft, 1990). 
porosity. (2) a phase of stylolitization with associated fracturing. (3) a second phase of leaching resulting in a widening of preexisting pores and fractures. (4) a late anhydritization. From fractures the anhydrite was spread in all directions, partly replacing the dolomite and partly filling the pores and cavities. Ultimately oil was introduced, visible oilstaining of the core has been observed in the interval between $2437.5 \mathrm{~m}$ and $2455 \mathrm{~m}$ b. KB. Below ca. $2455 \mathrm{~m}$ scattered droplets of oil are found (by examination of thin sections) in isolated pores and in stylolites. Above $2437.5 \mathrm{~m}$ to the top at ca. 2424 $\mathrm{m}$ only very few small, scattered pitch-like grains are seen in the pores.
In the present investigation we have analyzed rock samples by Instrumental Neutron Activation Analysis (INAA), Atomic Absorption Spectroscopy (AAS), and X-ray diffraction (XRD). An optical and electron microscopical examination of thin sections and fragments of the samples have been carried out. Palaeomagnetic measurements have been carried out on the same samples. These investigations allow us to make a geochemical characterization of the most abundant mineral phases, and to correlate the depositional environment with the trace element geochemistry. 


\section{Sample preparation and analytical procedures}

\section{Sampling}

The Løgumkloster-1 Well was drilled in 1980. The Ca-2 interval is ca. 43 meters long, and most of it is preserved as full core. Samples were obtained as 1" plugs. The contamination on the core surface has been avoided by sampling the interior of the core only.

\section{Thin and thick sections}

118 thin sections from 109 depth levels were prepared from the plugs for optical microscopy. Thick sections were prepared as fresh breaks of samples. Both thin and thick sections were studied by Scanning Electron Microscopy (a Phillips SEM-515).

\section{Instrumental Neutron Activation Analysis (INAA)}

48 of the 109 sample levels have been subjected to INAA analysis. Ca. 2 grams of rock were crushed in an agate mortar, which was rinsed in analytical grade hydrochloric acid followed by analytical grade acetone. Ca. $250 \mathrm{mg}$ of powder from the crushed and homogenized samples were transferred to polyethylene irradiation vials and subsequently irradiated for 4 hours in the heavy water reactor DR-3 at Ris $\varnothing$, Denmark. The thermal neutron flux at the sample position was $4 \times 10^{13} \mathrm{n} \mathrm{cm}^{-2} \mathrm{sec}^{-1}$. Samples were irradiated together with iron oxide $\left(\mathrm{Fe}_{2} \mathrm{O}_{3}\right)$ as an internal standard, and with gypsum rock standards as well as 4 samples of the drilling mud used during the drill operation (see Table 1).
The measurements were carried out on a high-purity germanium detector of an efficiency of $13.8 \%$, with $1.91 \mathrm{keV}$ as full width half maximum at the $1332 \mathrm{keV}$ line of Eu. A Nuclear Data 4096 multichannel pulseheight analyzer was used to collect the data. Samples were measured for 30 minutes in the first count after 7-9 days, and for 5-12 hours in the second count after 20-30 days.

The spectra were analyzed for 38 elements, among which generally 20 elements occurred in amounts above the detection limit. The elements for which concentrations were achieved are: $\mathrm{Na}, \mathrm{K}, \mathrm{Ca}, \mathrm{Sc}, \mathrm{Cr}$, $\mathrm{Fe}, \mathrm{Co}, \mathrm{Zn}, \mathrm{As}, \mathrm{Se}, \mathrm{Br}, \mathrm{Rb}, \mathrm{Sr}, \mathrm{Sb}, \mathrm{Cs}, \mathrm{Ba}$, $\mathrm{La}, \mathrm{Ce}, \mathrm{Nd}, \mathrm{Sm}, \mathrm{Eu}, \mathrm{Tb}, \mathrm{Yb}, \mathrm{Lu}, \mathrm{Hf}, \mathrm{Ta}$, $\mathrm{Au}, \mathrm{Hg}$, $\mathrm{Th}$, and $\mathrm{U}$. The uncertainty was generally better than $\pm 10 \%$ (one standard deviation), but some variation in the uncertainty was encountered depending on the bulk chemistry of the sample.

\section{Loss on ignition and wet chemical analysis}

Main elements were determined in the DGU-laboratory by wet chemistry and atomic absorption in aliquotes of the 48 samples subjected to INAA analysis. Firstly the $\mathrm{CO}_{3}^{2-}$-contents of the samples were determined by igniting a 1 gram dried sample for $1 / 2$ hour at $400^{\circ} \mathrm{C}$ followed by weighing; the sample was then reignited for 1 hour to $950^{\circ} \mathrm{C}$ followed by weighing. Secondly 0.5 grams of the samples were dried and dissolved in boiling $2 \mathrm{M}$ analytical grade nitric acid. Half of the filtrate of each sample was used for sulphate determination. This was done by precipitating the $\mathrm{SO}_{4}^{2-}$ with $\mathrm{BaCl}_{2}$ 
$\left(12.2 \%\right.$, analytical grade) in a surplus of $\mathrm{Cl}^{-}$ (analytical grade $\mathrm{HCl}$ added) followed by weighing the precipitate after drying. The other half of the filtrate was used for determination of $\mathrm{Ca}$ and $\mathrm{Mg}$ by atomic absorption, using the filtrate diluted to a concentration of ca. $1 \mu \mathrm{g} / \mathrm{g}$. The accuracy of these determinations were probably better than \pm $15 \%$ (one standard deviation), but some of the analysis were flawed by incomplete dissolution of the sample in nitric acid (the anhydrite was hard to get into solution). Also the high dilution factor necessary for the atomic absorption measurements may have impaired the accuracy of the results. The atomic absorption results should therefore be considered with some reservation.

\section{$X$-ray diffraction}

XRD was carried out at the Royal Vetenary and Agricultural University, Copenhagen, on powdered samples of whole rock samples, as well as on EDTA insoluble residue. The XRD analysis was carried out on a Phillips X-ray 1050 vertical diffractometer equipment, using $\mathrm{Co}-\mathrm{K}_{\alpha}$ radiation.

\section{Magnetic measurements}

The palaeomagnetic measurements were carried out on a United Scientific SCTA-100 helium cooled SQUID-magnetometer (Superconducting QUantum Interfering Device) situated at the Geophysical Institute, University of Copenhagen. 38 samples from the Løgumkloster-1 core were measured for NRM (Natural Remanent Magnetization).

The samples were then demagnetized in an alternating magnetic field, during which the samples were tumbled stocastically in 3 dimensions. During AC-demagnetization the ambient field was reduced to below 100 nT. Subsequently the samples were measured again on the SQUID-magnetometer. AC-demagnetization was performed at increasing field strengths: $50,100,200,300$ and $400 \mathrm{Oe}$. Following this, the samples were subjected to a DC-field of ca. 1890 Gauss for five minutes, after which the saturation magnetization was measured. Finally the susceptibility was measured on a KLY-2 Kappabridge. 
Table 1.

\begin{tabular}{|c|c|c|c|c|c|c|c|c|}
\hline Name & $\begin{array}{l}\text { Depth } \\
\text { m b. KB }\end{array}$ & $\begin{array}{c}\mathrm{Na} \\
\mu \mathrm{g} / \mathrm{g}\end{array}$ & $\begin{array}{c}\mathrm{K} \\
\mu \mathrm{g} / \mathrm{g}\end{array}$ & $\begin{array}{c}\mathrm{Ca} \\
\mu \mathrm{g} / \mathrm{g}\end{array}$ & $\begin{array}{c}\mathrm{Sc} \\
\mu \mathrm{g} / \mathrm{g}\end{array}$ & $\begin{array}{c}\mathrm{Cr} \\
\mu \mathrm{g} / \mathrm{g}\end{array}$ & $\begin{array}{c}\mathrm{Fe} \\
\mu \mathrm{g} / \mathrm{g}\end{array}$ & $\begin{array}{c}\text { Co } \\
\mu \mathrm{g} / \mathrm{g}\end{array}$ \\
\hline DGU-132 & 2424.0 & 537 & 831 & 236000 & 0.30700 & 3.430 & 702.0 & 1.1300 \\
\hline DGU-129 & 2425.3 & 396 & 121 & 244000 & 0.05820 & 0.828 & 144.0 & 0.1470 \\
\hline DGU-127 & 2425.9 & 450 & & 232000 & 0.08460 & 2.140 & 134.0 & 0.1720 \\
\hline DGU-124 & 2426.5 & 518 & & 177000 & 0.02930 & 1.850 & 41.9 & 0.1750 \\
\hline DGU-123 & 2427.4 & 4250 & & 265000 & 0.05290 & 2.290 & 89.2 & 0.1660 \\
\hline DGU-116 & 2428.8 & 453 & 239 & 261000 & 0.08580 & 2.960 & 198.0 & 0.2370 \\
\hline DGU-118 & 2429.1 & 493 & 155 & 176000 & 0.07040 & 1.570 & 109.0 & 0.1100 \\
\hline DGU-112 & 2430.4 & 433 & 288 & 277000 & 0.08710 & 1.360 & 177.0 & 0.0775 \\
\hline DGU-110 & 2431.1 & 273 & 149 & 126000 & 0.07440 & 1.350 & 53.1 & 0.0789 \\
\hline DGU-108 & 2432.9 & 273 & 151 & 87700 & 0.07680 & 1.570 & 57.4 & 0.0839 \\
\hline DGU-106 & 2433.5 & 299 & & 262000 & 0.01910 & 0.608 & 15.9 & 0.0323 \\
\hline DGU-104 & 2434.5 & 285 & & 86600 & 0.01950 & 0.768 & 35.3 & 0.0515 \\
\hline DGU-101 & 2435.5 & 345 & & 281000 & 0.01720 & 0.708 & 9.6 & 0.1260 \\
\hline DGU-99 & 2436.9 & 278 & & 73000 & 0.01500 & 0.718 & 16.5 & 0.0863 \\
\hline DGU-98 & 2437.5 & 296 & & 85600 & 0.01390 & 0.717 & 9.2 & 0.0662 \\
\hline DGU-96 & 2438.5 & 273 & & 84700 & 0.01480 & 0.705 & 18.9 & 0.0292 \\
\hline DGU-93 & 2440.0 & 266 & & 50100 & 0.01210 & 0.584 & 12.5 & 0.0547 \\
\hline DGU-91 & 2440.5 & 371 & & 90200 & 0.01310 & 0.525 & 7.4 & 0.0522 \\
\hline DGU-88 & 2441.9 & 291 & & 115000 & 0.00962 & 0.450 & & 0.0780 \\
\hline DGU-197 & 2442.2 & 434 & & 235000 & 0.01390 & 0.457 & 12.5 & 0.0221 \\
\hline DGU-191 & 2443.3 & 378 & & 251000 & 0.01370 & 0.505 & 25.3 & 0.0137 \\
\hline DGU-188 & 2443.7 & 231 & & 266000 & 0.00762 & 0.228 & 6.6 & 0.0135 \\
\hline DGU-190 & 2444.3 & 356 & & 242000 & 0.01190 & 0.326 & 8.9 & 0.0177 \\
\hline DGU-183 & 2445.3 & 379 & & 256000 & 0.00928 & 0.303 & 7.2 & 0.0140 \\
\hline DGU-182 & 2446.4 & 310 & & 289000 & 0.00903 & 0.234 & 21.3 & 0.0675 \\
\hline DGU-180 & 2447.0 & 461 & & 290000 & 0.00995 & 0.365 & 23.1 & 0.0817 \\
\hline DGU-179 & 2448.2 & & & 236000 & 0.01060 & 0.354 & 17.3 & 0.0264 \\
\hline DGU-175 & 2448.7 & 326 & & 263000 & 0.01240 & 0.365 & 11.6 & 0.0388 \\
\hline DGU-171 & 2450.0 & 336 & & 255000 & 0.01280 & 0.297 & 29.4 & 0.1050 \\
\hline DGU-168 & 2451.3 & 488 & & 260000 & 0.03330 & 0.566 & 50.7 & 0.0308 \\
\hline DGU-165 & 2452.0 & 456 & & 244000 & 0.03690 & 0.768 & 33.1 & 0.1260 \\
\hline DGU-163 & 2452.9 & 436 & 137 & 193000 & 0.03270 & 0.687 & 75.7 & 0.0786 \\
\hline DGU-162 & 2453.6 & 426 & & 237000 & 0.03640 & 0.740 & 25.5 & 0.0625 \\
\hline DGU-160 & 2454.2 & 406 & 181 & 266000 & 0.05300 & 1.070 & 95.3 & 0.1180 \\
\hline DGU-155 & 2455.3 & 393 & & 270000 & 0.02100 & 0.346 & 41.5 & 0.1740 \\
\hline DGU-157 & 2455.6 & 284 & 112 & 262000 & 0.03380 & 0.652 & 69.7 & 0.0833 \\
\hline DGU-149 & 2455.8 & 272 & & 171000 & 0.02300 & 0.647 & 29.6 & 0.0323 \\
\hline DGU-151 & 2456.0 & 226 & & 246000 & 0.02680 & 0.848 & 74.8 & 0.0733 \\
\hline DGU-144 & 2456.7 & 249 & & 263000 & 0.01400 & 0.457 & 155.0 & 0.1190 \\
\hline DGU-146 & 2457.1 & 317 & & 342000 & 0.02130 & 0.758 & 28.3 & 0.0520 \\
\hline DGU-143 & 2457.6 & 1900 & & 206000 & 0.01580 & 0.477 & 12.7 & 0.0314 \\
\hline DGU-138 & 2458.2 & 14200 & & 478000 & 0.03260 & 0.692 & 39.4 & 0.0651 \\
\hline DGU-135 & 2459.2 & 14700 & & 459000 & 0.03140 & 1.130 & 43.4 & 0.0533 \\
\hline DGU-134 & 2459.6 & 314 & 105 & 257000 & 0.05180 & 0.696 & 126.0 & 0.0324 \\
\hline DGU-204 & 2460.9 & 298 & & 260000 & 0.02680 & 1.490 & 520.0 & \\
\hline DGU-200 & 2462.8 & 310 & & 242000 & 0.29900 & 4.460 & 585.0 & 0.3160 \\
\hline DGU-201 & 2463.2 & 627 & 293 & 251000 & 0.33200 & 6.590 & 520.0 & 0.3300 \\
\hline DGU-199 & 2463.9 & 526 & 5020 & 306000 & 0.06990 & 0.753 & 232.0 & 0.1050 \\
\hline AVERAGE & & 1081 & 599 & 229290 & 0.04924 & 0.902 & 101.1 & 0.1114 \\
\hline $2455 \mathrm{M}$ & & 34100 & & 119000 & 4.73 & 69 & 22200 & 28.5 \\
\hline $2435 M$ & & 26800 & 15000 & 126000 & 4.39 & 86.4 & 21600 & 9.85 \\
\hline $2435 \mathrm{NK}$ & & 33700 & 11700 & 122000 & 3.99 & 85 & 21800 & 9.88 \\
\hline $2435 N$ & & 30900 & 15300 & 115000 & 5.2 & 43.8 & 1630 & 1.00 \\
\hline GYPA & & 63.2 & 260 & 102000 & 0.0859 & 0.735 & 310 & 0.106 \\
\hline GYPB & & 124 & 528 & 240000 & 0.15 & 1.61 & 472 & 0.216 \\
\hline GYPC & & 132 & 3320 & 236000 & 0.748 & 4.54 & 2960 & 1.05 \\
\hline GYPD & & 526 & 5020 & 215000 & 2.02 & 11.3 & 7580 & 2.12 \\
\hline CXR-1 & & 718 & & 14700 & 1.79 & 13.2 & 247000 & 7.96 \\
\hline CXR-2 & & 5580 & 14300 & 8400 & 6.62 & 38.1 & 18900 & 8.48 \\
\hline $\mathrm{MAG}-1$ & & 28600 & 27200 & 11400 & 16.8 & 104 & 47400 & 21.2 \\
\hline MAG-1 & & 29300 & 31700 & 10100 & 17.6 & 107 & 48800 & 22.2 \\
\hline MAG-1 & & 30000 & 30700 & 10600 & 17.7 & 106 & 49200 & 22.5 \\
\hline
\end{tabular}




\begin{tabular}{|c|c|c|c|c|c|c|c|c|}
\hline$\underset{\mu \mathrm{g} / \mathrm{g}}{\mathrm{Zn}}$ & $\begin{array}{c}\text { As } \\
\mu \mathrm{g} / \mathrm{g}\end{array}$ & $\begin{array}{c}\mathrm{Se} \\
\mu \mathrm{g} / \mathrm{g}\end{array}$ & $\begin{array}{c}\mathrm{Br} \\
\mu \mathrm{g} / \mathrm{g}\end{array}$ & $\begin{array}{c}\mathrm{Rb} \\
\mu \mathrm{g} / \mathrm{g}\end{array}$ & $\begin{array}{c}\mathrm{Sr} \\
\mu \mathrm{g} / \mathrm{g}\end{array}$ & $\begin{array}{c}\mathrm{Sb} \\
\mu \mathrm{g} / \mathrm{g}\end{array}$ & $\begin{array}{c}\mathrm{Cs} \\
\mu \mathrm{g} / \mathrm{g}\end{array}$ & $\begin{array}{c}\mathrm{Ba} \\
\mu \mathrm{g} / \mathrm{g}\end{array}$ \\
\hline 31.50 & 0.776 & 0.1820 & 11.60 & 2.660 & 1480 & & 0.1260 & 17.1 \\
\hline 5.85 & 0.201 & & 8.47 & 0.418 & 11100 & & 0.0239 & 64.4 \\
\hline 6.98 & 0.287 & 0.1100 & 5.90 & & 7510 & & 0.0326 & 24.9 \\
\hline 32.10 & 0.520 & & 6.02 & & 923 & & & \\
\hline 23.10 & 0.884 & 0.0666 & 7.21 & 0.598 & 729 & & 0.0174 & \\
\hline 18.00 & 1.050 & 0.1820 & 3.56 & 0.709 & 12000 & & 0.0286 & 40.8 \\
\hline 10.30 & 0.284 & 0.1110 & 4.81 & 0.673 & 2030 & & 0.0310 & \\
\hline 5.98 & 0.406 & 0.2710 & 8.60 & 0.771 & 873 & & 0.0329 & \\
\hline 7.10 & 0.137 & 0.0519 & 4.43 & 0.477 & 409 & & 0.0187 & 14.2 \\
\hline 7.20 & 0.198 & 0.0558 & 4.37 & & 453 & & 0.0171 & 26.8 \\
\hline 21.10 & 0.118 & & 4.54 & & 2610 & & & 22.5 \\
\hline 9.77 & 0.127 & 0.0565 & 4.75 & & 813 & & & \\
\hline 12.30 & & & 7.70 & & 789 & & & 22.5 \\
\hline 14.70 & 0.081 & & 6.10 & & 548 & 0.0135 & & \\
\hline 6.74 & 0.082 & & 6.44 & & 331 & & & \\
\hline 5.44 & 0.170 & & 6.26 & & 6320 & 0.0122 & & 42.6 \\
\hline 7.21 & 0.113 & & 4.70 & & 571 & 0.0077 & & \\
\hline 1.59 & 0.083 & & 5.64 & & 1410 & 0.0079 & & \\
\hline 8.51 & & & 6.30 & & 415 & & & \\
\hline 4.86 & & & 5.59 & & 127 & & & \\
\hline 1.71 & 0.267 & 0.0764 & 4.66 & & 2690 & & 0.0078 & 16.3 \\
\hline 15.00 & & & 3.57 & & 1340 & & & \\
\hline 8.09 & & & 9.93 & & 258 & & 0.0070 & \\
\hline 4.51 & & & 11.30 & & 614 & & & \\
\hline 8.41 & 0.109 & 0.0671 & 5.47 & 0.234 & 1310 & & 0.0053 & \\
\hline 13.50 & 0.133 & & 11.90 & & 4670 & & & 33.3 \\
\hline 7.87 & & & & & 5130 & & 0.0072 & 172.0 \\
\hline 6.60 & & & 6.03 & & 806 & & 0.0071 & \\
\hline 20.40 & 0.344 & & 6.12 & & 7780 & & 0.0050 & 66.5 \\
\hline 8.22 & 0.140 & 0.0482 & 11.10 & & 697 & & 0.0191 & \\
\hline 14.60 & 0.261 & 0.0540 & 12.80 & & 2010 & & 0.0125 & \\
\hline 11.30 & 0.284 & 0.0920 & 8.81 & 0.194 & 6010 & & 0.0161 & 29.0 \\
\hline 5.72 & & & 10.10 & & 10500 & & 0.0111 & 50.5 \\
\hline 15.70 & 0.352 & 0.1310 & 7.77 & & 8330 & & 0.0178 & 39.9 \\
\hline 16.10 & 0.206 & 0.0633 & 8.53 & & 2340 & & 0.0100 & 28.9 \\
\hline 3.10 & 0.306 & 0.1560 & 5.68 & & 870 & 0.0221 & 0.0191 & \\
\hline 1.42 & 0.261 & 0.1680 & 6.46 & & 5160 & & 0.0076 & 33.3 \\
\hline 2.14 & 0.451 & 0.6130 & 4.16 & & 414 & 0.0254 & 0.0113 & 12.3 \\
\hline 2.14 & 0.551 & 1.4200 & 4.66 & & 982 & 0.0499 & & \\
\hline 3.39 & 0.198 & 0.1140 & 5.72 & & 401 & & 0.0103 & 23.4 \\
\hline 10.40 & & 0.0403 & 14.10 & & 102 & & 0.0086 & \\
\hline 14.70 & & & 15.90 & & 5620 & & 0.0202 & \\
\hline 13.80 & & & 15.90 & & 5050 & & & \\
\hline \multirow[t]{2}{*}{1.19} & 0.197 & 0.0686 & 7.72 & & 730 & & 0.0247 & \\
\hline & 0.609 & & 5.64 & & 1110 & 0.0512 & & \\
\hline 1.12 & 0.975 & 0.6970 & 2.29 & 1.900 & 640 & 0.0814 & 0.1130 & 15.6 \\
\hline 0.50 & 0.379 & 1.0300 & 2.60 & 2.420 & 863 & 0.0222 & 0.1310 & \\
\hline 0.64 & 3.390 & 0.1180 & 0.83 & 26.500 & 1270 & 0.2130 & 0.0379 & 110.0 \\
\hline 9.63 & 0.414 & 0.2325 & 7.08 & 3.130 & 2690 & 0.0460 & 0.0270 & 41.2 \\
\hline 890 & 11 & 0.59 & 130 & 59.9 & 3950 & 1.4 & 4.81 & 697 \\
\hline 580 & 5.45 & 0.22 & 220 & 56.7 & 1570 & 0.668 & 4.73 & 450 \\
\hline 567 & 5.24 & & 205 & 54.2 & 1570 & 0.822 & 4.47 & 326 \\
\hline 168 & 5.09 & 82.6 & 190 & 27.3 & 71.3 & 0.83 & 13 & 318 \\
\hline 1.39 & 0.283 & & 0.135 & 1.29 & 1060 & 0.0253 & 0.069 & \\
\hline 1.45 & 0.327 & & 0.32 & 2.28 & 1170 & 0.0243 & 0.0795 & \\
\hline 13.7 & 2.9 & 1.25 & 1.59 & 9 & 3170 & 0.164 & 0.303 & 55 \\
\hline 15.3 & 3.39 & 2.04 & 0.825 & 24.6 & 1520 & 0.213 & 1.16 & 110 \\
\hline 649 & 422 & 15.8 & & & 380 & 107 & 2.99 & 1370 \\
\hline 482 & 25 & 0.564 & 3.29 & 78.7 & 198 & 40.6 & 5.14 & 2050 \\
\hline 128 & 10.7 & 0.974 & 224 & 158 & 162 & 1.01 & 8.4 & 553 \\
\hline 89.5 & 10.4 & & 232 & 148 & 155 & 0.798 & 8.75 & 770 \\
\hline 112 & 10.4 & & 225 & 153 & 172 & 0.856 & 8.67 & 562 \\
\hline
\end{tabular}




\begin{tabular}{|c|c|c|c|c|c|c|c|c|}
\hline $\begin{array}{c}\mathrm{La} \\
\mu \mathrm{g} / \mathrm{g}\end{array}$ & $\begin{array}{c}\mathrm{Ce} \\
\mu \mathrm{g} / \mathrm{g}\end{array}$ & $\begin{array}{c}\mathrm{Nd} \\
\mu \mathrm{g} / \mathrm{g}\end{array}$ & $\begin{array}{c}\mathrm{Sm} \\
\mu \mathrm{g} / \mathrm{g}\end{array}$ & $\begin{array}{c}\mathrm{Eu} \\
\mu \mathrm{g} / \mathrm{g}\end{array}$ & $\begin{array}{c}\mathrm{Tb} \\
\mu \mathrm{g} / \mathrm{g}\end{array}$ & $\begin{array}{c}\mathrm{Yb} \\
\mu \mathrm{g} / \mathrm{g}\end{array}$ & $\begin{array}{c}\mathrm{Lu} \\
\mu \mathrm{g} / \mathrm{g}\end{array}$ & $\begin{array}{c}\mathrm{Hf} \\
\mu \mathrm{g} / \mathrm{g}\end{array}$ \\
\hline 1.090 & 3.290 & 2.020 & 0.1200 & 0.0437 & 0.0340 & 0.0570 & & 0.1400 \\
\hline 0.462 & 1.190 & 0.976 & & 0.0236 & 0.0141 & 0.0414 & & 0.0208 \\
\hline 0.346 & 1.420 & 1.440 & 0.0231 & 0.0247 & 0.0249 & 0.0775 & & 0.0585 \\
\hline 0.231 & 0.808 & 1.040 & 0.0273 & 0.0109 & 0.0114 & 0.0259 & 0.0061 & 0.0113 \\
\hline 0.286 & 1.320 & 1.240 & & 0.0184 & 0.0164 & 0.0526 & $0.0 \mathrm{~L} 08$ & 0.0269 \\
\hline 0.205 & 1.070 & 1.200 & & 0.0089 & & & & 0.0388 \\
\hline 0.249 & 0.927 & 0.689 & & 0.0124 & & 0.0182 & & 0.0237 \\
\hline 0.195 & 0.909 & 1.070 & & 0.0100 & 0.0102 & 0.0297 & & 0.0303 \\
\hline 0.174 & 1.220 & 1.320 & & 0.0063 & & 0.0138 & & 0.0353 \\
\hline 0.187 & 1.370 & 1.540 & & 0.0090 & & 0.0134 & & 0.0372 \\
\hline 0.083 & 1.050 & 0.855 & 0.0834 & 0.0043 & & 0.0117 & & 0.0049 \\
\hline 0.907 & 0.700 & I.070 & & 0.0035 & & 0.0142 & & 0.0051 \\
\hline 0.658 & 0.642 & 1.110 & & 0.0046 & & 0.0134 & & \\
\hline 0.691 & 0.746 & 1.050 & & 0.0032 & & 0.0159 & 0.0023 & \\
\hline 0.521 & 0.548 & 0.975 & & 0.0045 & & 0.0213 & 0.0010 & \\
\hline 0.963 & 1.110 & 1.550 & 0.1130 & 0.0046 & & 0.0133 & 0.0056 & \\
\hline 0.343 & 0.482 & 0.956 & & 0.0035 & & 0.0147 & & \\
\hline 0.323 & 0.660 & 1.360 & & 0.0044 & & 0.0160 & 0.0008 & \\
\hline 0.216 & 0.475 & 1.260 & & 0.0048 & & 0.0146 & 0.0010 & \\
\hline 0.075 & 0.459 & 0.417 & & & & & & 0.0067 \\
\hline 0.077 & 0.568 & 0.660 & & 0.0036 & 0.0030 & & 0.0059 & 0.0036 \\
\hline 0.083 & 0.401 & 0.371 & & 0.0021 & & & & \\
\hline 0.079 & 0.612 & 0.684 & & 0.0033 & & & 0.0052 & \\
\hline 0.082 & 0.892 & 0.747 & & 0.0027 & & & & \\
\hline 0.045 & 0.204 & 0.340 & & 0.0027 & & & & 0.0032 \\
\hline \multirow[t]{2}{*}{0.112} & 0.317 & & & 0.0051 & 0.0068 & & & \\
\hline & 0.772 & 0.766 & & 0.0040 & 0.0032 & & & \\
\hline 0.058 & 0.374 & 0.414 & & 0.0032 & & & & \\
\hline 0.051 & 0.184 & 0.322 & & 0.0031 & & & & 0.0045 \\
\hline 0.128 & 0.803 & 0.606 & & 0.0065 & & & & 0.0095 \\
\hline 0.088 & 0.501 & 0.552 & & 0.0065 & & & & 0.0061 \\
\hline 0.141 & 0.574 & 0.641 & & 0.0072 & 0.0048 & & & 0.0307 \\
\hline 0.110 & 0.563 & 0.637 & & 0.0060 & & & & 0.0067 \\
\hline 0.107 & 0.690 & 0.796 & & 0.0063 & & & & 0.0449 \\
\hline 0. 105 & 0.581 & 0.825 & & 0.0046 & & & 0.0046 & 0.0065 \\
\hline 0.125 & 0.780 & 0.821 & & 0.0052 & & 0.0235 & & 0.0116 \\
\hline 0.118 & 0.691 & 0.682 & & 0.0067 & 0.0059 & 0.0191 & & \\
\hline 0.137 & 1.230 & 1.050 & & 0.0061 & & & & 0.0169 \\
\hline 0.084 & 0.761 & 0.855 & & 0.0047 & & & & \\
\hline 0.150 & 1.300 & 1.240 & & 0.0064 & 0.0040 & & 0.0044 & 0.0079 \\
\hline \multirow[t]{3}{*}{0.168} & 0.896 & 0.828 & & 0.0060 & 0.0052 & 0.0126 & & 0.0048 \\
\hline & 1.440 & 1.710 & & 0.0114 & & & & \\
\hline & 1.260 & 1.400 & & 0.0111 & & & & 0.0180 \\
\hline 0.207 & 0.937 & 0.794 & & 0.0077 & & & & 0.0299 \\
\hline 0.206 & 0.696 & & & & & & & \\
\hline 0.150 & 2.820 & 2.290 & 0.0756 & 0.0296 & 0.0222 & & & 0.1020 \\
\hline 0.060 & $2.600)$ & 1.850 & 0.0968 & 0.0292 & 0.0249 & & & 0.1210 \\
\hline 4.390 & 1.010 & 0.904 & & 0.0066 & & 0.4220 & 0.0516 & 0.0328 \\
\hline 0.339 & 0.934 & 0.998 & 0.0770 & 0.0088 & 0.0127 & 0.0428 & 0.0083 & 0.0290 \\
\hline 15.1 & 31.9 & 14.7 & 2.73 & 0.561 & 0.409 & 0.509 & 0.163 & 1.75 \\
\hline 13 & 27.6 & 14.4 & 4.22 & 0.491 & 0.316 & 0.85 & 0.149 & 1.56 \\
\hline 12.7 & 26.9 & 13.2 & 6.55 & 0.468 & 0.323 & 1.07 & 0.133 & 1.48 \\
\hline 13.3 & 57.1 & 8.82 & 25.6 & 1.34 & 1.12 & 0.807 & 0.0455 & 8.03 \\
\hline 0.217 & 0.42 & 1.03 & & 0.016 & & 0.0599 & 0.0029 & 0.0435 \\
\hline 0.485 & 1.03 & 1.23 & 0.05 & 0.0254 & & 0.0311 & 0.0020 & 0.0709 \\
\hline 2.87 & 5.94 & 4.76 & & 0.105 & 0.0506 & 0.153 & 0.0219 & 0.26 \\
\hline 4.39 & 9.77 & 4.7 & 0.91 & 0.182 & 0.168 & 0.422 & 0.0516 & 0.434 \\
\hline 8.75 & 20.1 & & 2.01 & 0.674 & 1.87 & 2.28 & 0.27 & 0.96 \\
\hline 25.2 & 48.4 & & 0.369 & 0.775 & 0.58 & 1.9 & 0.235 & 6.46 \\
\hline 40.8 & 83.2 & 40.8 & 7.15 & 1.44 & 0.978 & 2.64 & 0.337 & 3.4 \\
\hline 42.4 & 81.6 & 45.5 & 7.01 & 1.51 & 1.06 & 2.68 & 0.393 & 3.42 \\
\hline 42.7 & 80.5 & 42.7 & 7.35 & 1.54 & 1.04 & 2.76 & 0.398 & 3.58 \\
\hline
\end{tabular}




\begin{tabular}{|c|c|c|c|}
\hline $\begin{array}{c}\mathrm{Ta} \\
\mu \mathrm{g} / \mathrm{g}\end{array}$ & $\begin{array}{c}\mathrm{Hg} \\
\mu \mathrm{g} / \mathrm{g}\end{array}$ & $\begin{array}{c}\text { Th } \\
\mu \mathrm{g} / \mathrm{g}\end{array}$ & $\begin{array}{c}\mathrm{U} \\
\mu \mathrm{g} / \mathrm{g}\end{array}$ \\
\hline \multirow[t]{8}{*}{0.0323} & 2.490 & 0.2920 & 3.29 \\
\hline & 2.860 & 0.0708 & 1.18 \\
\hline & 2.160 & 0.0892 & 2.65 \\
\hline & 0.783 & 0.0392 & 1.50 \\
\hline & 0.519 & 0.0538 & 2.58 \\
\hline & 0.569 & 0.0707 & 2.58 \\
\hline & 0.642 & 0.0700 & 1.62 \\
\hline & 0.489 & 0.0551 & 1.79 \\
\hline \multirow[t]{37}{*}{0.0064} & 1.730 & 0.0479 & 3.13 \\
\hline & 1.970 & 0.0547 & 3.34 \\
\hline & 14.200 & 0.0141 & 1.62 \\
\hline & 1.440 & 0.0187 & 1.89 \\
\hline & 0.875 & 0.0138 & 1.66 \\
\hline & 0.491 & 0.0151 & 2.03 \\
\hline & 0.465 & 0.0124 & 1.47 \\
\hline & 0.482 & 0.0137 & 3.36 \\
\hline & 0.707 & 0.0115 & 1.58 \\
\hline & 0.891 & 0.0151 & 1.81 \\
\hline & 0.727 & & 1.53 \\
\hline & 0.131 & 0.0096 & \\
\hline & 0.188 & 0.0091 & \\
\hline & 0.106 & & \\
\hline & 0.141 & & \\
\hline & 0.187 & 0.0070 & \\
\hline & 0.251 & 0.0072 & \\
\hline & 0.249 & 0.0114 & \\
\hline & 0.321 & & \\
\hline & 1.900 & 0.0060 & \\
\hline & 0.215 & & \\
\hline & 0.285 & 0.0217 & 2.05 \\
\hline & 0.296 & 0.0243 & \\
\hline & 0.225 & 0.0270 & \\
\hline & 0.826 & 0.0246 & \\
\hline & 0.201 & 0.0443 & \\
\hline & 0.297 & 0.0154 & 1.46 \\
\hline & 0.183 & 0.0226 & 1.93 \\
\hline & 0.227 & 0.0170 & 15.00 \\
\hline & 0.417 & 0.0203 & 3.24 \\
\hline & 0.382 & 0.0094 & 2.14 \\
\hline & 0.261 & 0.0235 & 3.61 \\
\hline & 0.341 & 0.0098 & 2.33 \\
\hline & 0.765 & 0.0295 & 3.22 \\
\hline & 0.626 & 0.0196 & 3.30 \\
\hline & 0.375 & 0.0576 & 1.76 \\
\hline & & & 1.25 \\
\hline 0.0232 & 0.124 & 0.2070 & \\
\hline \multirow[t]{2}{*}{0.0326} & 0.099 & 0.2620 & \\
\hline & 0.111 & 1.5200 & \\
\hline 0.0236 & 0.941 & 0.0801 & 2.64 \\
\hline 0.536 & 0.092 & 0.0918 & 3.20 \\
\hline 0.494 & & 4.50 & \\
\hline 0.461 & & 4.33 & 3.28 \\
\hline \multirow[t]{4}{*}{7.040} & 3.090 & 6.96 & \\
\hline & 0.664 & 0.0991 & 0.10 \\
\hline & 0.477 & 0.165 & 0.19 \\
\hline & 1.170 & 0.586 & 0.68 \\
\hline \multirow[t]{2}{*}{0.128} & 0.110 & 1.51 & \\
\hline & 4.970 & 2.9 & 31.70 \\
\hline 0.837 & 4.050 & 8.83 & 3.93 \\
\hline 1.200 & & 12 & 2.88 \\
\hline 1.240 & & 12.2 & \\
\hline 1.340 & 0.366 & 12.3 & \\
\hline
\end{tabular}

Table 1. Instrumental Neutron Activation Analysis (INAA) of Ca-2 samples, commercial standard rocks, and samples of the drilling mud. The approximate uncertainties (one standard deviation) of the determinations are $\pm 10 \%$. 


\section{Results}

The results of the INAA analysis are listed in Table 1. The standard rocks included are MAG-1 marine mud from U.S. Geologic Survey (Govindaraju, 1984; Abbey, 1983), GXR-1 and GXR-2 soils (Abbey, 1983), and GYP-A, GYP-B, GYP-C, and GYP-D gypsum samples (Kocman \& Woodger, 1986). Additionally four drilling mud samples from two levels (2435 $\mathrm{m}$ and $2455 \mathrm{~m}$ ) have been analyzed. The mud was obtained both from the surface of the core and from inside fractures formed by the relief of the pressure during the coring. Only concentrations above the detection limits are given in Table 1.

In Table 2 are given the recommended values of the standard rocks (Govindaraju, 1984; Abbey, 1983; Kocman \& Woodger, 1986). A reasonable agreement exists between these values and our determinations

\begin{tabular}{|c|c|c|c|c|c|c|c|c|c|c|}
\hline Name & $\begin{array}{c}\mathrm{Na} \\
\mu \mathrm{g} / \mathrm{g}\end{array}$ & $\begin{array}{c}\mathrm{K} \\
\mu \mathrm{g} / \mathrm{g}\end{array}$ & $\begin{array}{c}\mathrm{Ca} \\
\mu \mathrm{g} / \mathrm{g}\end{array}$ & $\begin{array}{c}\mathrm{Sc} \\
\mu \mathrm{g} / \mathrm{g}\end{array}$ & $\begin{array}{c}\mathrm{Cr} \\
\mu \mathrm{g} / \mathrm{g}\end{array}$ & $\begin{array}{c}\mathrm{Fe} \\
\mu \mathrm{g} / \mathrm{g}\end{array}$ & $\begin{array}{c}\text { Co } \\
\mu \mathrm{g} / \mathrm{g}\end{array}$ & $\begin{array}{c}\mathrm{Zn} \\
\mu \mathrm{g} / \mathrm{g}\end{array}$ & $\begin{array}{c}\text { As } \\
\mu \mathrm{g} / \mathrm{g}\end{array}$ & $\begin{array}{c}\mathrm{Se} \\
\mu \mathrm{g} / \mathrm{g}\end{array}$ \\
\hline GYPA & 66 & 174 & 235000 & 0.09 & 2 & 350 & 0.2 & 7 & 0.19 & \\
\hline GYPB & 155 & 415 & 234000 & 0.16 & 2 & 490 & 0.7 & 7 & 0.20 & \\
\hline GYPC & 163 & 2980 & 217000 & 0.80 & 4 & 2800 & 1.2 & 15 & 2.4 & \\
\hline GYPD & 519 & 4480 & 202000 & 2.0 & 9 & 7550 & 2.4 & 16 & 3.0 & \\
\hline GXR-1 & 816 & 498 & 8860 & 1.7 & 10 & 250000 & 9.3 & 740 & 460 & 18.50 \\
\hline GXR-2 & 5420 & 13600 & 8790 & 6.8 & 37 & 18400 & 9.0 & 500 & 31 & 0.74 \\
\hline \multirow[t]{2}{*}{ MAG-1 } & 29000 & 30900 & 9860 & 17 & 105 & 48800 & 20 & 135 & & \\
\hline & $\begin{array}{c}\mathrm{Br} \\
\mu \mathrm{g} / \mathrm{g}\end{array}$ & $\begin{array}{c}\mathrm{Rb} \\
\mu \mathrm{g} / \mathrm{g}\end{array}$ & $\begin{array}{c}\mathrm{Sr} \\
\mu \mathrm{g} / \mathrm{g}\end{array}$ & $\begin{array}{c}\mathrm{Sb} \\
\mu \mathrm{g} / \mathrm{g}\end{array}$ & $\begin{array}{c}\text { Cs } \\
\mu \mathrm{g} / \mathrm{g}\end{array}$ & $\begin{array}{c}\mathrm{Ba} \\
\mu \mathrm{g} / \mathrm{g}\end{array}$ & $\begin{array}{c}\mathrm{La} \\
\mu \mathrm{g} / \mathrm{g}\end{array}$ & $\begin{array}{c}\mathrm{Ce} \\
\mu \mathrm{g} / \mathrm{g}\end{array}$ & $\begin{array}{c}\mathrm{Nd} \\
\mu \mathrm{g} / \mathrm{g}\end{array}$ & $\begin{array}{c}\mathrm{Sm} \\
\mu \mathrm{g} / \mathrm{g}\end{array}$ \\
\hline GYPA & 0.5 & 0.8 & 930 & 0.040 & 0.15 & 28 & 0.2 & 0.7 & & 0.04 \\
\hline GYPB & 0.4 & 4 & 1180 & 0.024 & 0.20 & 25 & 0.6 & 1.24 & & 0.07 \\
\hline GYPC & 1.7 & 11 & 2950 & 0.160 & 0.41 & 53 & 3.0 & 5 & & 0.45 \\
\hline GYPD & 1.3 & 25 & 1520 & 0.280 & 1.3 & 106 & 5.0 & 9 & & 0.83 \\
\hline GXR-1 & 0.4 & 29 & 280 & 125 & 4.0 & 560 & 6.1 & 19 & & \\
\hline GXR-2 & 3.0 & 86 & 160 & 48 & 5.0 & 2000 & 25 & 50 & & 3.30 \\
\hline \multirow[t]{2}{*}{ MAG-1 } & & 150 & 140 & 1.00 & 8.6 & 480 & 41 & 86 & 41 & 8.10 \\
\hline & $\begin{array}{c}\mathrm{Eu} \\
\mu \mathrm{g} / \mathrm{g}\end{array}$ & $\begin{array}{c}\mathrm{Tb} \\
\mu \mathrm{g} / \mathrm{g}\end{array}$ & $\begin{array}{c}Y b \\
\mu \mathrm{g} / \mathrm{g}\end{array}$ & & & $\begin{array}{c}\text { Hf } \\
\mu \mathrm{g} / \mathrm{g}\end{array}$ & $\begin{array}{c}\mathrm{Ta} \\
\mu \mathrm{g} / \mathrm{g}\end{array}$ & $\begin{array}{c}\mathrm{Hg} \\
\mu \mathrm{g} / \mathrm{g}\end{array}$ & $\begin{array}{c}\text { Th } \\
\mu \mathrm{g} / \mathrm{g}\end{array}$ & $\begin{array}{c}\mathrm{U} \\
\mu \mathrm{g} / \mathrm{g}\end{array}$ \\
\hline GYPA & 0.06 & & 0.02 & & & 0.26 & & & 0.10 & 0.10 \\
\hline GYPB & 0.07 & & 0.03 & & 07 & 0.32 & & & 0.15 & 0.23 \\
\hline GYPC & 0.12 & & 0.17 & & 50 & 0.36 & & & 0.51 & 0.72 \\
\hline GYPD & 0.17 & & 0.44 & & 67 & 0.60 & 0.15 & & 1.3 & 0.65 \\
\hline GXR-1 & 0.68 & & 1.80 & & & 1.10 & 0.20 & 3.9 & 2.3 & 35 \\
\hline GXR-2 & 0.80 & & 2.20 & & & 9.60 & 0.76 & 3.2 & 8.3 & 3.00 \\
\hline MAG-1 & 1.50 & 1.00 & 2.60 & & & 3.60 & 1.10 & & 12.5 & 2.80 \\
\hline
\end{tabular}

Table 2. The recommended values of the standard rocks analyzed by INAA. From Gorvindaraju (1984) Abbey (1983) and Kocman \& Woodger (1986). The values obtained in the present work are listed in Table 1. 
Constituents and amount used of the drilling mud

Magcogel (Wyoming Bentonite, montmorillonite)

Caustic Soda $(\mathrm{NaOH})$

Soda Ash $\left(\mathrm{Na}_{2} \mathrm{CO}_{3}, 10 \mathrm{H}_{2} \mathrm{O}\right)$

Lime $(\mathrm{CaO})$

Drispac (polymer)

Salt $(\mathrm{NaCl})$

Tannathin (lignite, C)

Magco-cfl (Cr-free lignite)

Salt Gel (attapulgite clay)

Poly Sal (polymer with additives)

$\mathrm{XC}$-Polymer (polysaccharoses)

$\mathrm{CaCO}_{3}$

650 sacks

62 sacks

75 sacks

47 sacks

50 sacks

6090 sacks

378 sacks

165 sacks

1017 sacks

500 sacks

59 sacks

747 sacks

Table 3. The constituencies of the drilling mud as reported by the operator (Dansk Boreselskab, 1981).

of the standard rocks in Table 1. In Table 3 is given the drilling mud constituencies as reported by the operator (Dansk Boreselskab, 1981).

The results of the atomic absorption analysis are listed in Table 4. The dolomite content is calculated on the assumption that all $\mathrm{Mg}$ occurs in dolomite and that the $\mathrm{Ca} / \mathrm{Mg}$ ratio of the dolomite is unity, which was confirmed by XRD measurements on bulk samples. The amount of anhydrite quoted in Table 4 is calculated assuming that all $\mathrm{SO}_{4}^{2-}$ is present as anhydrite. Celestine $\left(\mathrm{SrSO}_{4}\right)$ is known to be present in this depth interval (Stentoft, 1989), but only in insignificant amounts. The amount of halite $(\mathrm{NaCl})$ listed in Table 4 is calculated on the assumption that all $\mathrm{Na}$ occurs as $\mathrm{NaCl}$, thus the calculated amount of halite includes the minute amounts of sylvite sometimes present. As mentioned earlier, some samples were incompletely dissolved, wherefore a somewhat larger than usual uncertainty must be expected for the results in Table 4 .

The palaeomagnetic results are listed in Table 5. Only intensities and inclinations are listed as the core is recovered without recorded orientation (declination).

Typical examples of the behavior of the samples are shown in Figure 6. On average about $86 \%$ of the magnetization remains after 50 Oe of AC-demagnetization of the Løgumkloster samples, and 24\% after 100 Oe. The magnetization of some of the samples have been completely removed after 100 Oe of demagnetization. We consider the results after 50 Oe treatment for the most reliable.

Porosity, permeability, grain density, and facies type have also been determined and are reported elsewhere (Stentoft, 1990), the results are summarized in Table 6 .

The INAA abundance data for $\mathrm{Th}, \mathrm{U}$, and $\mathrm{K}$ have been compared with natural gamma ray (NGT) well logging data and core gamma scanning (CGS) data. The core gamma scanning (CGS) was performed in the laboratory of DGU. An extract from the CGS-log is shown in Table 7, where the concentrations are extracted in the same levels at which the INAA were performed. The detection limits for the CGS-log were ca. $0.24 \mu \mathrm{g} / \mathrm{g}$ for $\mathrm{Th}, 0.14 \mu \mathrm{g} / \mathrm{g}$ for $\mathrm{U}$, and $230 \mu \mathrm{g} / \mathrm{g}$ for $\mathrm{K}$. Most of the measurements were quite close to the detection limit. The concentrations of $\mathrm{K}, \mathrm{Th}$, and $\mathrm{U}$ determined by NGT are shown in Figure 7 . Also the NGT measurements are uncomfortably close to the detection limit. It is not considered worthwhile to make a detailed comparison between the INAA results with the NGT and CGS data, due to the vast difference in spatial (depth) resolution of the methods, INAA has a spatial resolution of less than a centimeter, whereas CGS has a resolution of ca. $10 \mathrm{~cm}$, and NGT of ca. 0.5 $\mathrm{m}$. Other workers have found significant discrepancies between NGT and INAA determined values for $\mathrm{K}$ at abundances below ca. $10 \mathrm{mg} / \mathrm{g}$ (Gendron et al., 1988). 


\begin{tabular}{|c|c|c|c|c|c|c|c|c|c|c|c|c|c|}
\hline $\begin{array}{l}\text { Depth } \\
\text { m }\end{array}$ & Name & $\begin{array}{r}\mathrm{Na} \\
\mu \mathrm{g} / \mathrm{g}\end{array}$ & $\begin{array}{c}\mathrm{K} \\
\mu \mathrm{g} / \mathrm{g}\end{array}$ & $\begin{array}{l}\mathrm{Ca} \\
\%\end{array}$ & $\begin{array}{l}\mathrm{Mg} \\
\%\end{array}$ & $\begin{array}{c}\text { SO4 } \\
\%\end{array}$ & $\begin{array}{c}\mathrm{Cl} \\
\mathrm{mg} / \mathrm{g}\end{array}$ & $\begin{array}{c}\mathrm{CO} 3 \\
\%\end{array}$ & $\begin{array}{c}\text { DOL } \\
\%\end{array}$ & $\begin{array}{c}\text { ANH } \\
\%\end{array}$ & $\begin{array}{c}\text { UND } \\
\%\end{array}$ & $\underset{\%}{\text { Sum }}$ & $\begin{array}{c}\text { HAL } \\
\%\end{array}$ \\
\hline 2424.0 & -132 & 723 & 193 & 22 & 11 & 7 & 1.00 & 57 & 87 & 13 & 0.3 & 101 & 0.16 \\
\hline 2425.3 & -129 & 549 & 130 & 23 & 10 & 11 & 0.70 & 48 & 74 & 24 & 0.2 & 98 & 0.12 \\
\hline 2425.9 & -127 & 642 & 131 & 22 & 12 & 7 & 0.50 & 58 & 89 & 11 & 0.4 & 100 & 0.08 \\
\hline 2426.5 & -124 & 555 & 113 & 24 & 8 & 20 & 0.50 & 41 & 63 & 35 & 0.1 & 99 & 0.08 \\
\hline 2427.4 & -123 & 4538 & 127 & 23 & 10 & 11 & 6.25 & 49 & 76 & 22 & 0.0 & 98 & 1.03 \\
\hline 2428.8 & -116 & 610 & 127 & 25 & 9 & 14 & 0.45 & 44 & 67 & 35 & 0.1 & 102 & 0.07 \\
\hline 2429.1 & -118 & 625 & 108 & 24 & 9 & 15 & 0.50 & 43 & 65 & 35 & 0.0 & 100 & 0.08 \\
\hline 2430.4 & -112 & 517 & 120 & 23 & 9 & 14 & 0.75 & 42 & 65 & 31 & 0.0 & 95 & 0.12 \\
\hline 2431.1 & -110 & 366 & 72 & 23 & 11 & 8 & 0.35 & 54 & 82 & 16 & 0.0 & 98 & 0.06 \\
\hline 2432.9 & -108 & 412 & 116 & 23 & 10 & 10 & 0.50 & 49 & 76 & 19 & 0.2 & 95 & 0.08 \\
\hline 2433.5 & -106 & 438 & 79 & 25 & 9 & 13 & 1.38 & 42 & 65 & 32 & 0.1 & 97 & 0.23 \\
\hline 2434.5 & -104 & 367 & 59 & 23 & 9 & 16 & 0.35 & 42 & 65 & 33 & 0.1 & 98 & 0.06 \\
\hline 2435.5 & -101 & 427 & 65 & 23 & 10 & 12 & 0.50 & 48 & 74 & 25 & 1.0 & 99 & 0.08 \\
\hline 2436.9 & -99 & 382 & 73 & 23 & 10 & 11 & 0.50 & 50 & 77 & 20 & 0.0 & 97 & 0.08 \\
\hline 2437.5 & -98 & 494 & 71 & 20 & 12 & 6 & 0.50 & 61 & 94 & 8 & 0.0 & 102 & 0.08 \\
\hline 2438.5 & -96 & 376 & 70 & 23 & 9 & 13 & 0.45 & 46 & 71 & 24 & 4.0 & 99 & 0.07 \\
\hline 2440.0 & -93 & 360 & 58 & 23 & 10 & 9 & 0.40 & 51 & 79 & 15 & 0.0 & 94 & 0.07 \\
\hline 2440.5 & -91 & 474 & 66 & 24 & 12 & 12 & 0.40 & 59 & 90 & 23 & 0.0 & 113 & 0.07 \\
\hline 2441.9 & -88 & 426 & 106 & 23 & 11 & 7 & 0.50 & 54 & 83 & 14 & 0.0 & 97 & 0.08 \\
\hline 2442.2 & -197 & 676 & 50 & 22 & 13 & 2 & 1.00 & 63 & 96 & 3 & 0.0 & 99 & 0.16 \\
\hline 2443.3 & -191 & 552 & 44 & 23 & 10 & 12 & 0.63 & 48 & 74 & 26 & 0.2 & 100 & 0.10 \\
\hline 2443.7 & -188 & 386 & 81 & 26 & 5 & 24 & 0.69 & 26 & 40 & 58 & 0.0 & 98 & 0.11 \\
\hline 2444.3 & -190 & 547 & 82 & 22 & 12 & 6 & 1.10 & 59 & 90 & 9 & 0.0 & 99 & 0.18 \\
\hline 2445.3 & -183 & 528 & 75 & 23 & 10 & 9 & 0.79 & 50 & 77 & 21 & 0.2 & 98 & 0.13 \\
\hline 2446.4 & -182 & 813 & 100 & 24 & 5 & 25 & 0.50 & 24 & 37 & 51 & 0.4 & 87 & 0.08 \\
\hline 2447.0 & -180 & 1205 & 296 & 27 & 9 & 13 & 1.47 & 44 & 68 & 32 & 0.0 & 100 & 0.24 \\
\hline 2448.2 & -179 & 19000 & 96 & 22 & 11 & 8 & 31.10 & 54 & 83 & 14 & 0.0 & 97 & 5.13 \\
\hline 2448.7 & -175 & 438 & 65 & 24 & 9 & 14 & 0.56 & 42 & 65 & 33 & 0.0 & 98 & 0.09 \\
\hline 2450.0 & -171 & 451 & 73 & 28 & 7 & 20 & 0.56 & 35 & 54 & 55 & 0.3 & 109 & 0.09 \\
\hline 2451.3 & -168 & 625 & 99 & 24 & 10 & 11 & 1.00 & 49 & 75 & 26 & 0.1 & 102 & 0.16 \\
\hline 2452.0 & -165 & 614 & 108 & 23 & 11 & 7 & 1.00 & 55 & 84 & 17 & 0.0 & 101 & 0.16 \\
\hline 2452.9 & -163 & 2940 & 614 & 26 & 7 & 19 & 0.75 & 33 & 51 & 41 & 0.1 & 92 & 0.12 \\
\hline 2453.6 & -162 & 645 & 131 & 22 & 12 & 7 & 0.90 & 59 & 91 & 9 & 0.0 & 100 & 0.15 \\
\hline 2454.2 & -160 & 593 & 175 & 29 & 6 & 25 & 0.70 & 32 & 49 & 64 & 0.0 & 113 & 0.12 \\
\hline 2455.3 & -155 & 493 & 113 & 24 & 7 & 19 & 0.70 & 36 & 55 & 43 & 0.0 & 98 & 0.12 \\
\hline 2455.6 & -157 & 479 & 105 & 24 & 8 & 16 & 1.50 & 39 & 60 & 39 & 0.0 & 99 & 0.25 \\
\hline 2455.8 & -149 & 403 & 69 & 22 & 11 & 7 & 0.58 & 56 & 86 & 11 & 0.0 & 97 & 0.10 \\
\hline 2456.0 & -151 & 348 & 64 & 23 & 11 & 8 & 0.45 & 56 & 86 & 15 & 0.0 & 101 & 0.07 \\
\hline 2456.7 & -144 & 431 & 53 & 24 & 7 & 19 & 0.63 & 36 & 55 & 42 & 0.1 & 97 & 0.10 \\
\hline 2457.1 & -146 & 484 & 63 & 22 & 11 & 5 & 0.63 & 55 & 85 & 13 & 0.0 & 98 & 0.10 \\
\hline 2457.6 & -143 & 2525 & 90 & 21 & 13 & 1 & 4.00 & 63 & 97 & 2 & 0.0 & 99 & 0.66 \\
\hline 2458.2 & -138 & 16500 & 89 & 22 & 12 & 3 & 27.40 & 61 & 93 & 9 & 0.0 & 102 & 4.52 \\
\hline 2459.2 & -135 & 504 & 96 & 23 & 9 & 15 & 0.70 & 45 & 69 & 28 & 0.0 & 98 & 0.12 \\
\hline 2459.6 & -134 & 471 & 113 & 24 & 9 & 14 & 0.70 & 46 & 71 & 28 & 0.0 & 99 & 0.12 \\
\hline 2460.9 & -204 & 503 & 88 & 24 & 7 & 20 & 0.63 & 35 & 54 & 43 & 0.1 & 97 & 0.10 \\
\hline 2462.8 & -200 & 1000 & 299 & 22 & 10 & 11 & 1.60 & 49 & 75 & 20 & 0.9 & 96 & 0.26 \\
\hline 2463.2 & -201 & 65 & 22 & 20 & 9 & 13 & 0.84 & 43 & 65 & 28 & 2.2 & 95 & 0.14 \\
\hline 2463.9 & -199 & 27 & 11 & 27 & 1 & 33 & 0.20 & 5 & 8 & 87 & 1.7 & 96 & 0.03 \\
\hline
\end{tabular}

Table 4. Abundances determined by atomic absorption spectroscopy and loss on ignition. The dolomite content is calculated on the assumption that all $\mathrm{Mg}$ occurs in dolomite and that the $\mathrm{Ca} / \mathrm{Mg}$-ratio of the dolomite is 1.0 (i.c. dolomite $=\mathrm{CaMgCO}_{3}$ ). The anhydrite content is calculated on the assumption that all $\mathrm{SO}_{4}^{2-}$ occurs as anhydrite. The amount of halite $(\mathrm{NaCl})$ listed in Table 4 is calculated on the assumption that all $\mathrm{Na}$ occurs as $\mathrm{NaCl}$. The uncertainties are probably better than $\pm 20 \%$ (one standard deviation), although the results may occasionally be affected by incomplete dissolution of the samples prior to analysis. 


\begin{tabular}{|c|c|c|c|c|c|c|c|c|}
\hline Name & $\begin{array}{c}\text { Depth } \\
\text { m b.KB }\end{array}$ & $\begin{array}{c}\mathrm{J}-\mathrm{O} \\
\$\end{array}$ & $\begin{array}{c}\text { INC-O } \\
\text { DEG }\end{array}$ & $\begin{array}{c}\mathrm{J}-50 \\
\$\end{array}$ & $\begin{array}{c}\text { INC-50 } \\
\text { DEG }\end{array}$ & $\begin{array}{c}\mathrm{J}-\text { sat } \\
\$\end{array}$ & $\begin{array}{l}\mathbf{K} \\
*\end{array}$ & $\begin{array}{l}{[\mathrm{Fe}]} \\
\mu \mathrm{g} / \mathrm{g}\end{array}$ \\
\hline LOE1D & 2424.00 & 102 & 53.0 & 89 & 63.0 & 18540 & -6.1 & 702 \\
\hline LOE4D & 2425.36 & 161 & 61.5 & 137 & 65.3 & 25975 & -6.5 & 144 \\
\hline LOE6D & 2426.55 & 54 & 57.3 & 50 & 79.4 & 13052 & -8.8 & 42 \\
\hline LOE12D & 2429.34 & 25 & 74.4 & 18 & 59.8 & 5684 & -10.9 & 109 \\
\hline LOE21D & 2433.85 & 12 & 71.4 & 13 & 60.3 & 3721 & -9.8 & 16 \\
\hline LOE25D & 2435.95 & 11 & 81.5 & 7 & 77.0 & 5308 & -9.7 & 10 \\
\hline LOE27D & 2437.01 & 45 & -13.4 & 6 & 35.1 & 3507 & -10.6 & 9 \\
\hline LOE29D & 2438.14 & 5 & -32.6 & 4 & 8.1 & 2884 & -10.8 & 19 \\
\hline LOE37D & 2442.00 & 27 & 15.6 & 15 & 33.1 & 3558 & -8.5 & 13 \\
\hline LOE4OD & 2443.65 & 15 & 51.0 & 26 & 31.3 & 5786 & -10.1 & 25 \\
\hline LOE43D & 2445.28 & 8 & 42.8 & 12 & 18.4 & 3039 & -11.2 & 7 \\
\hline LOE47D & 2447.30 & 9 & 51.2 & 5 & 62.7 & 2679 & -12.9 & 23 \\
\hline LOE50D & 2448.67 & 22 & 51.5 & 17 & 78.5 & 5917 & -10.6 & 17 \\
\hline LOE52D & 2449.85 & 17 & 65.6 & 10 & 74.2 & 3679 & -11.7 & 12 \\
\hline LOE54D & 2450.75 & 5 & 76.0 & 7 & 32.4 & 2967 & -11.9 & 29 \\
\hline LOE58D & 2453.00 & 13 & 76.8 & 11 & 83.3 & 4993 & -12.4 & 76 \\
\hline LOE60D & 2453.70 & 29 & 64.2 & 18 & 67.7 & 6215 & -12.1 & 26 \\
\hline LOE62D & 2454.65 & 71 & 77.3 & 53 & 68.7 & 9887 & -11.8 & 95 \\
\hline LOE65D & 2456.45 & 27 & 51.7 & 28 & 43.6 & 7533 & -8.7 & 155 \\
\hline LOE67D & 2457.20 & 24 & 77.6 & 17 & 72.0 & 4828 & -9.3 & 28 \\
\hline LOE70D & 2458.65 & 73 & 75.2 & 27 & 66.1 & 5422 & -11.0 & 39 \\
\hline LOE73D & 2460.00 & 63 & 46.1 & 40) & 60.7 & 12461 & -11.3 & 520 \\
\hline LOE75D & 2461.10 & 43 & 64.1 & 39 & 37.9 & 11689 & -10.8 & \\
\hline LOE77D & 2462.25 & 360 & 48.9 & 230 & 46.1 & 40243 & -3.4 & 585 \\
\hline LOE79D & 2463.06 & 117 & 72.1 & 76 & 72.7 & 25109 & -7.5 & 520 \\
\hline LOE84D & 2465.92 & 3 & 54.7 & 2 & 0.0 & 1556 & -12.9 & \\
\hline LOE86D & 2467.10 & 2 & 0.0 & 2 & -33.7 & 1479 & -13.1 & \\
\hline LOE88D & 2465.27 & 3 & 74.2 & 5 & 30.2 & 1521 & -13.1 & \\
\hline LOE90D & 2469.15 & 3 & 29.1 & 7 & 8.9 & 1639 & -13.2 & \\
\hline LOE92D & 2470.20 & 6 & 68.9 & 4 & 59.0 & 1863 & -12.6 & \\
\hline LOE94D & 2471.07 & 15 & 85.4 & 14 & 53.4 & 3124 & -12.1 & \\
\hline LOE96D & 2472.13 & 12 & 42.3 & 6 & 78.5 & 2485 & -13.0 & \\
\hline LOE98D & 2472.90 & 6 & 5.4 & 4 & -80.0 & 4058 & -12.9 & \\
\hline LOE101D & 2473.98 & 8 & 68.0 & 5 & 40.3 & 2024 & -12.6 & \\
\hline LOE $103 \mathrm{D}$ & 2474.80 & 8 & 39.8 & 6 & 55.9 & 2918 & -12.5 & \\
\hline LOE I05D & 2475.85 & 21 & 70.4 & 12 & 68.0 & 2971 & -12.6 & \\
\hline LOE107D & 2476.98 & 66 & 63.7 & 44 & 77.5 & 2929 & -12.1 & \\
\hline LOE109D & 2477.85 & 4 & 62.2 & 4 & 26.3 & 2035 & -12.9 & \\
\hline
\end{tabular}

$\$$ : palaeomagnetic intensity in $10^{-8} \mathrm{emu} / 10 \mathrm{cc}$

$*$ : susceptibility in units of $\mathrm{SI} * 10^{-6} / 10 \mathrm{cc}$

Table 5. Results of the palacomagnetic investigations. Only intensities and inclinations are listed as the core was recovered without recorded declination (azimuth). O stands for Natural Remnant Magnetization, ' $50^{\text {' are the }}$ same samples measured after $50 \mathrm{Oe}$ of AC-demagnetization. $\mathrm{J}$ is the length of the magnetic vector in $10^{-8}$ $\mathrm{cmu} / 10 \mathrm{~cm}^{3}$ (the actual sample volume was ca. $18.2 \mathrm{~cm}^{3}$ ). INC is inclination, $\mathrm{K}$ is susceptibility (in $10^{-6}$ SI-units $\left./ 10 \mathrm{~cm}^{3}\right),[\mathrm{Fe}]$ is the concentration of $\mathrm{Fe}$ in the nearest INAA sample (from Table 1). The threshold limit of the intensity measurements is ca. $1.010^{-8} \mathrm{emu} / 10 \mathrm{~cm}^{3}$. 
A

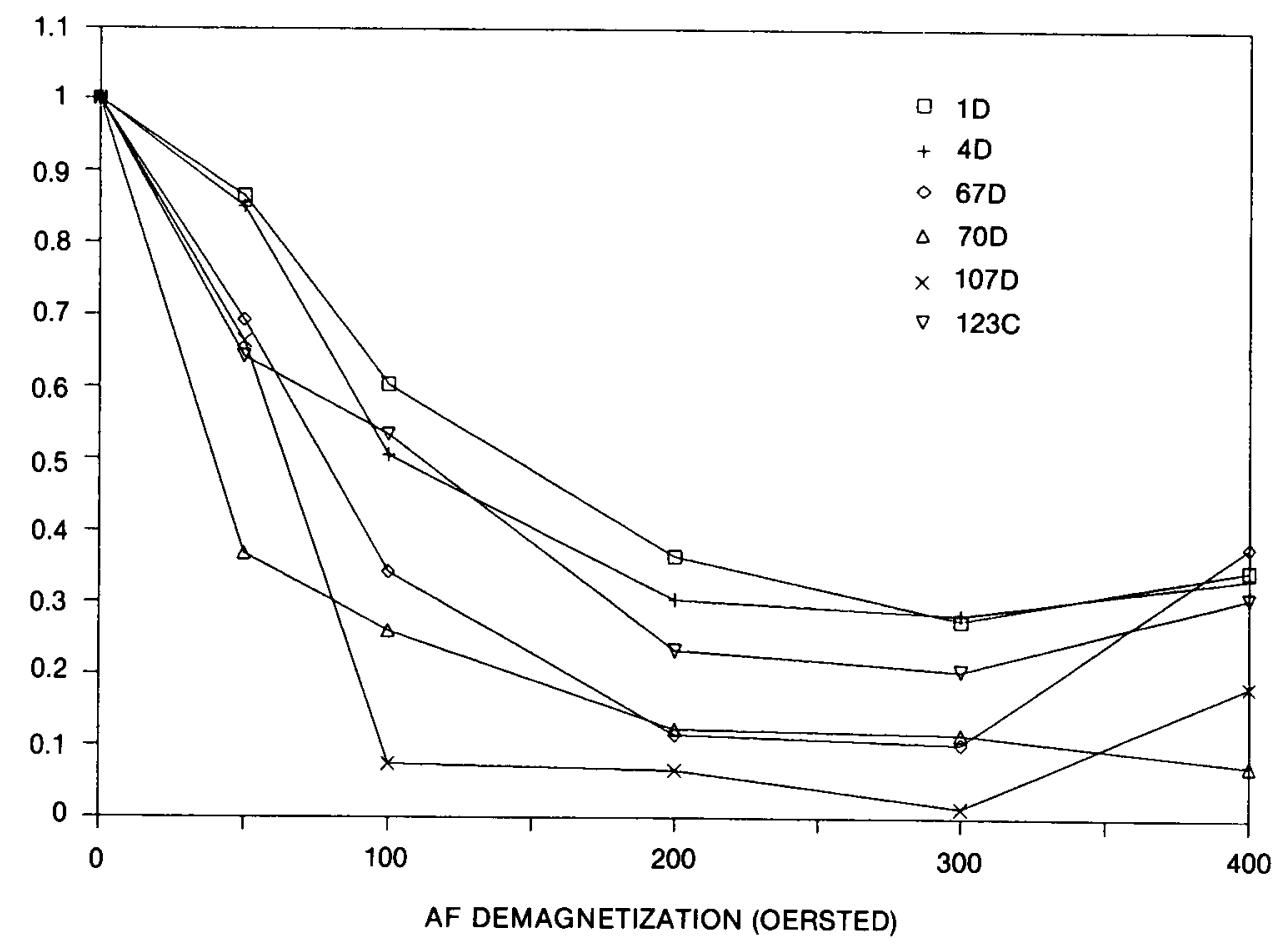

B

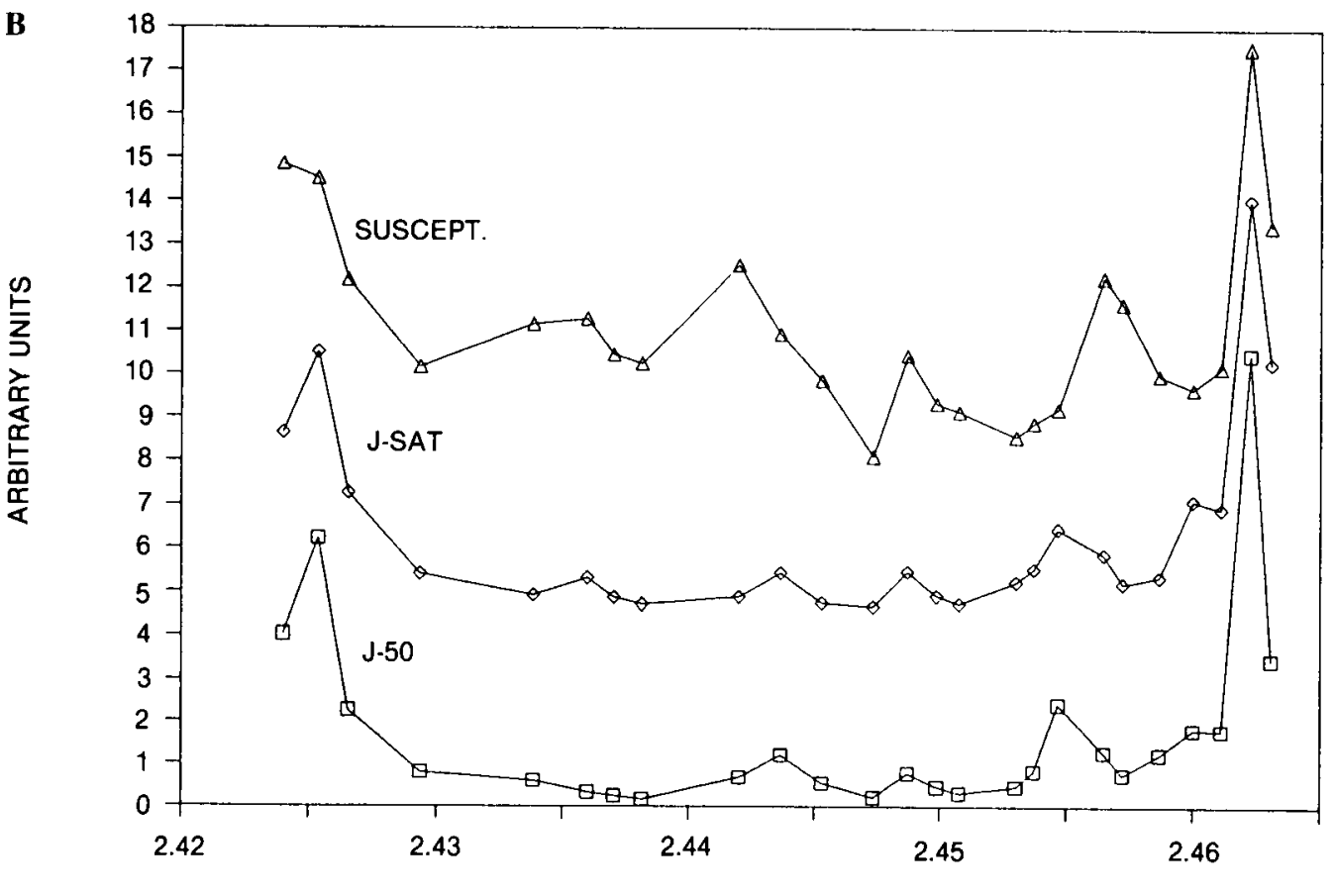

Stratigraphical Depth (m b. KB) (Thousands) 


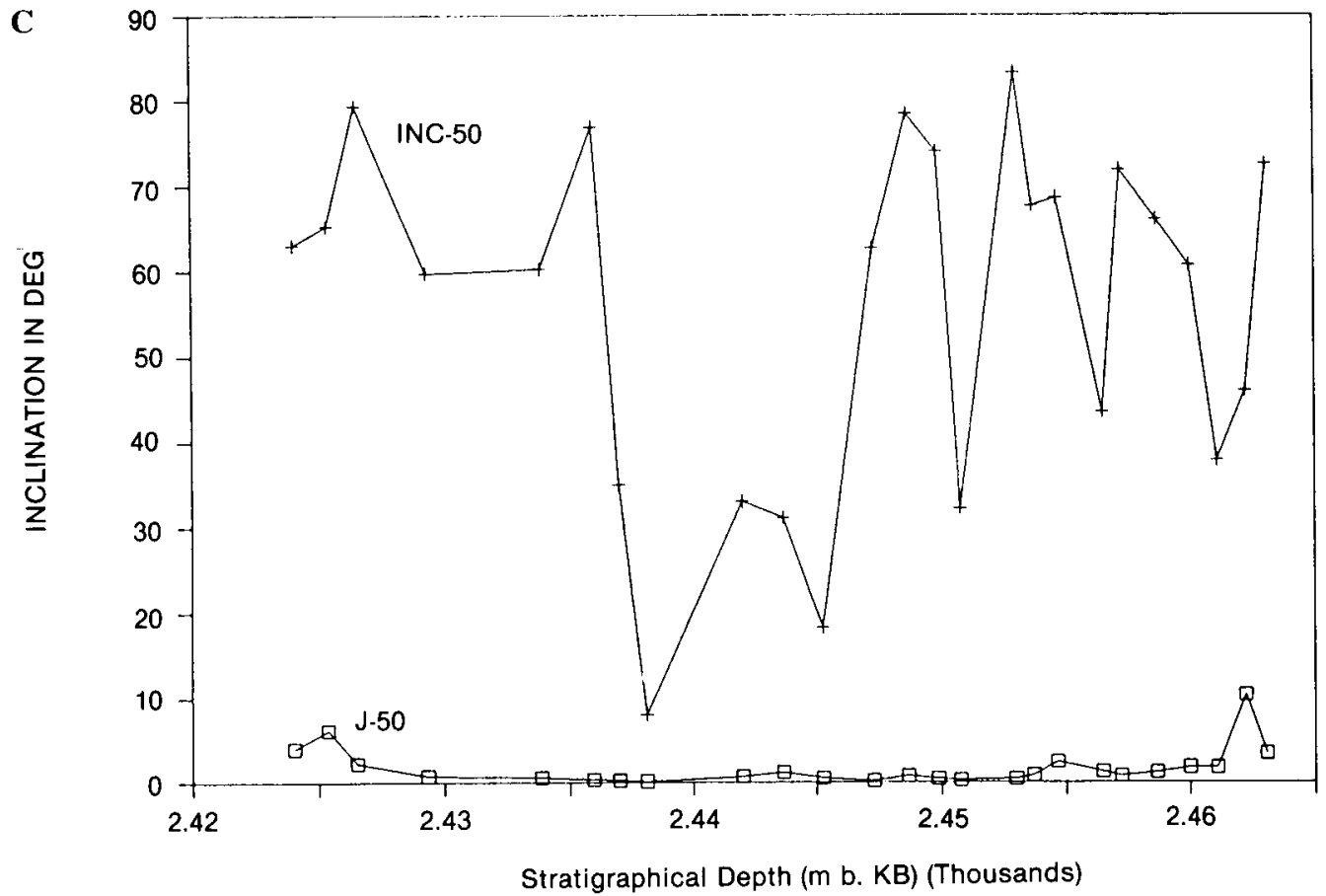

Fig. 6. A: Typical examples of the demagnetization behavior of the samples. On average about $86 \%$ of the magnetization remains after 50 Oe of AC-demagnetization of the Løgumkloster samples, and $24 \%$ after 100 Oe. The magnetization of some of the samples have been completely removed after 100 Oe of demagnetization. We consider the results after 50 Oe treatment for the most reliable. B: Paleomagnetic intensity (after 50 Oe AC-demagnetization), saturation magnetization, and susceptibility as a function of stratigraphical depth. C: Inclination (after $50 \mathrm{Oe}$ ) and intensity (after $50 \mathrm{Oe}$, arbitrary units) as a function of stratigraphical depth. 


\begin{tabular}{|c|c|c|c|c|c|c|}
\hline $\begin{array}{l}\text { Depth } \\
\text { m }\end{array}$ & Name & $\underset{\%}{\text { POR }}$ & $\begin{array}{c}\text { PERM } \\
\text { mD }\end{array}$ & $\begin{array}{l}\text { DEN } \\
\mathrm{g} / \mathrm{cm}^{3}\end{array}$ & $\begin{array}{c}\text { STYL. } \\
\mathrm{m}^{-1}\end{array}$ & $\begin{array}{c}\text { Facies } \\
\text { Type }\end{array}$ \\
\hline 2424.0 & -132 & 20.4 & & 2.88 & 12 & B \\
\hline 2425.3 & -129 & 12.2 & & 2.92 & 12 & B \\
\hline 2425.9 & -127 & 7.5 & & 2.90 & 12 & B \\
\hline 2426.5 & -124 & 17.3 & 171 & 2.94 & & B \\
\hline 2427.4 & -123 & 6.0 & 2.18 & & 3 & B \\
\hline 2428.8 & -116 & 15.9 & 44 & 2.93 & 7 & B \\
\hline 2429.1 & -118 & 4.5 & 0.01 & 2.91 & 23 & B \\
\hline 2430.4 & -112 & 12.3 & 2.08 & 2.90 & 22 & B \\
\hline 2431.1 & -110 & 15.5 & 4.51 & 2.89 & 29 & B \\
\hline 32.9 & -108 & 21.0 & 29 & 2.88 & 15 & B \\
\hline 33.5 & -106 & 17.3 & & 2.92 & 10 & A \\
\hline 34.5 & -104 & 12.6 & 0.90 & 2.91 & 6 & A \\
\hline 35.5 & -101 & 19.3 & 33 & 2.90 & & A \\
\hline 2436.9 & -99 & 21.6 & 41 & 2.89 & & A \\
\hline 37.5 & -98 & 21.5 & 17 & 2.8 & 3 & A \\
\hline 138.5 & -96 & 18.9 & 2.83 & 2.90 & 2 & A \\
\hline 40.0 & -93 & 15.4 & 45 & 2.88 & 0 & A \\
\hline 2440.5 & -91 & 24.0 & 2.43 & 2.88 & 0 & A \\
\hline 2441.9 & -88 & 19.2 & 137 & 2.8 & ( & A \\
\hline 2442.2 & -197 & 20.9 & 10.6 & 2.9 & i & A \\
\hline 2443.3 & -191 & 17.7 & 1.51 & 2. & 2 & $\mathrm{~A} / \mathrm{B}$ ? \\
\hline 2443.7 & -188 & 5.8 & 0.10 & 2.9 & 2 & B \\
\hline 2444.3 & -190 & 24.3 & 170 & 2.88 & 1 & $\mathrm{~A} / \mathrm{B}$ ? \\
\hline 2445.3 & -183 & 21.2 & 8.12 & 2.89 & 1 & B \\
\hline 2446.4 & -182 & 1.8 & 0.02 & 2.9 & 2 & B \\
\hline 2447.0 & -180 & 5.9 & 1.35 & 2.9 & 4 & B \\
\hline 2448.2 & -179 & 15.2 & 90 & & 1 & B \\
\hline 2448.7 & -175 & 8.9 & & 2.9 & 1 & B \\
\hline 2450.0 & -171 & 12.3 & 12 & 2.94 & 7 & B \\
\hline 2451.3 & -168 & 3.8 & & 2.76 & 2 & B \\
\hline 2452.0 & -165 & 7.0 & 1.20 & 2.8 & 9 & B \\
\hline 2452.9 & 163 & 3.8 & 0.05 & 2.93 & 9 & B \\
\hline & 162 & 3.8 & & 0 & 14 & B \\
\hline 2454.2 & 160 & 2.6 & 0.01 & 2.9 & 3 & B \\
\hline 2455.3 & -155 & 3.1 & 3.61 & 2.93 & 16 & B \\
\hline 2455.6 & -157 & 4.5 & 0.02 & 2.91 & 16 & B \\
\hline 2455.8 & -149 & 6.4 & 0.13 & 2.90 & 16 & B \\
\hline 2456.0 & 151 & 13.3 & 1.30 & 2.8 & 9 & B \\
\hline 2456.7 & 144 & 13.0 & 20 & & 9 & B \\
\hline 2457.1 & 146 & 24.9 & 14 & & 14 & A \\
\hline 2457.6 & -143 & 18.3 & 493 & 2.83 & 14 & $\mathrm{~A} / \mathrm{B}$ ? \\
\hline 2458.2 & -138 & 14.9 & 128 & & 6 & $\mathrm{~A} / \mathrm{B} ?$ \\
\hline 2459.2 & -135 & 6.3 & 0.20 & 2.90 & 30 & B \\
\hline & -134 & 9.2 & 0.45 & 2.90 & 30 & B \\
\hline & & 4.9 & 0.02 & & 9 & B \\
\hline & & 6.7 & 0.08 & & 8 & $\mathrm{~A} / \mathrm{B}$ ? \\
\hline 2463.2 & & 7.7 & 0.26 & 2.88 & 3 & $\mathrm{~A} / \mathrm{B}$ ? \\
\hline 2463.9 & -199 & 2.6 & 0.02 & 2.94 & 3 & $\mathrm{~A} / \mathrm{B}$ ? \\
\hline & & & & 2.90 & & \\
\hline
\end{tabular}

Table 6. Porosity, permeability, grain density, stylolite density, and facies type of the samples listed in Table 1. From Stentoft (1990). Facies type A: an oolitic shoal facies. Facies type B: a lagoonal carbonate facies.

\begin{tabular}{cccc}
\hline $\begin{array}{c}\text { Depth } \\
\mathrm{m}\end{array}$ & $\begin{array}{c}\mathrm{K} \\
\mu \mathrm{g} / \mathrm{g}\end{array}$ & $\begin{array}{c}\mathrm{U} \\
\mu \mathrm{g} / \mathrm{g}\end{array}$ & $\begin{array}{c}\mathrm{Th} \\
\mu \mathrm{g} / \mathrm{g}\end{array}$ \\
\hline 2424.1 & 600 & 1.7 & \\
2425.3 & 400 & 1.9 & \\
2425.8 & & 1.8 & \\
2429.1 & 400 & 1.6 & \\
2430.4 & 400 & 1.7 & \\
2431.1 & 300 & 1.8 & \\
2432.9 & & 1.9 & \\
2452.9 & & 1.5 & \\
2454.2 & 400 & 1.0 & \\
2455.6 & 400 & 1.7 & \\
2456.0 & 300 & 2.6 & \\
2459.6 & 400 & 2.0 & \\
2463.2 & 1200 & 6.9 & 0.4 \\
2463.9 & 300 & 2.2 & \\
\hline
\end{tabular}

Table 7. Extract from the Core Gamma Scanning log (CGS) listing the concentrations of $\mathrm{Th}, \mathrm{U}$, and $\mathrm{K}$. The detection limits were ca. $0.24 \mu \mathrm{g} / \mathrm{g}$ for Th, 0.14 $\mu \mathrm{g} / \mathrm{g}$ for $\mathrm{U}$, and $230 \mu \mathrm{g} / \mathrm{g}$ for $\mathrm{K}$. Note that most of the measurements were quite close to the detection limit.

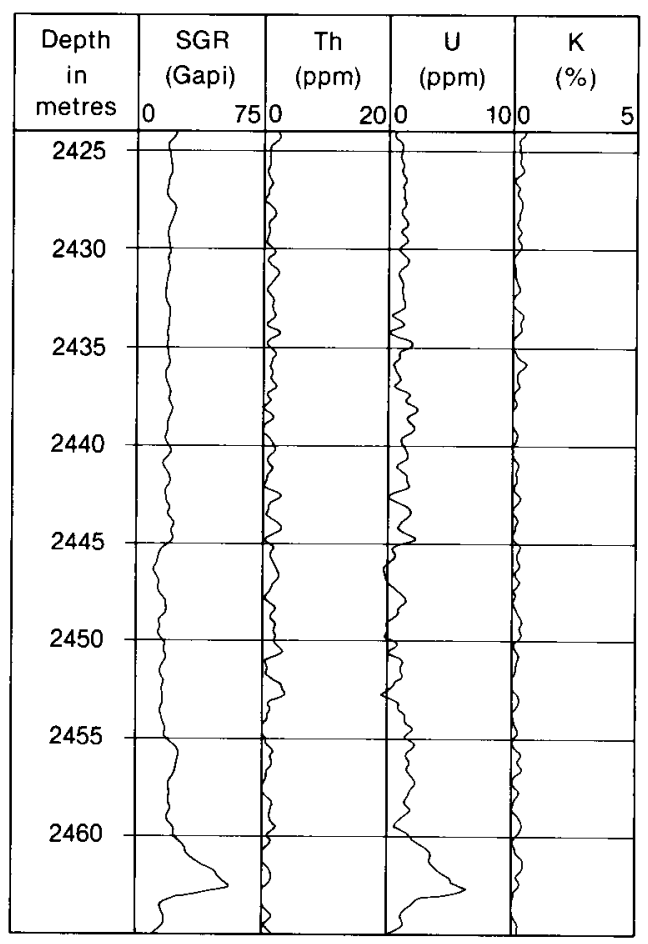

Fig. 7. The concentrations of $K, T h$, and $U$ in the Ca-2 interval of the Løgumkloster-1 well determined by NGT (Natural Gamma Ray Tool) wirelog. The concentrations are quite close to the detection limit. 


\section{Discussion}

The commonly used procedure of calculating inter-element correlation coefficients has been abandoned in this study as it was found that even statistically significant correlations very often were meaningless when the actual cross plot were contemplated.

\section{Anhydrite}

Strontium is a constituent of celestine $\left(\mathrm{SrSO}_{4}\right)$. Therefore it would be expected that samples, where celestine crystals have been observed in the corresponding thin sections, would be particularly rich in Sr. In Figure 8, which shows Sr concentration versus depth, these samples are marked with a "c". No correlation is, however, seen between $\mathrm{Sr}$ and "c", suggesting that the bulk of the $\mathrm{Sr}$ must be situated in other minerals than celestine. $\mathrm{Sr}^{2+}$ is known to substitute for $\mathrm{Ca}^{2+}$ in anhydrite (Usdowski, 1973). Strontium may also substitute for $\mathrm{Ca}$ in dolomite (Goldschmidt, 1954; Usdowski, 1973; Veizer, 1978), although we do not consider this to be as important as substitution in anhydrite.

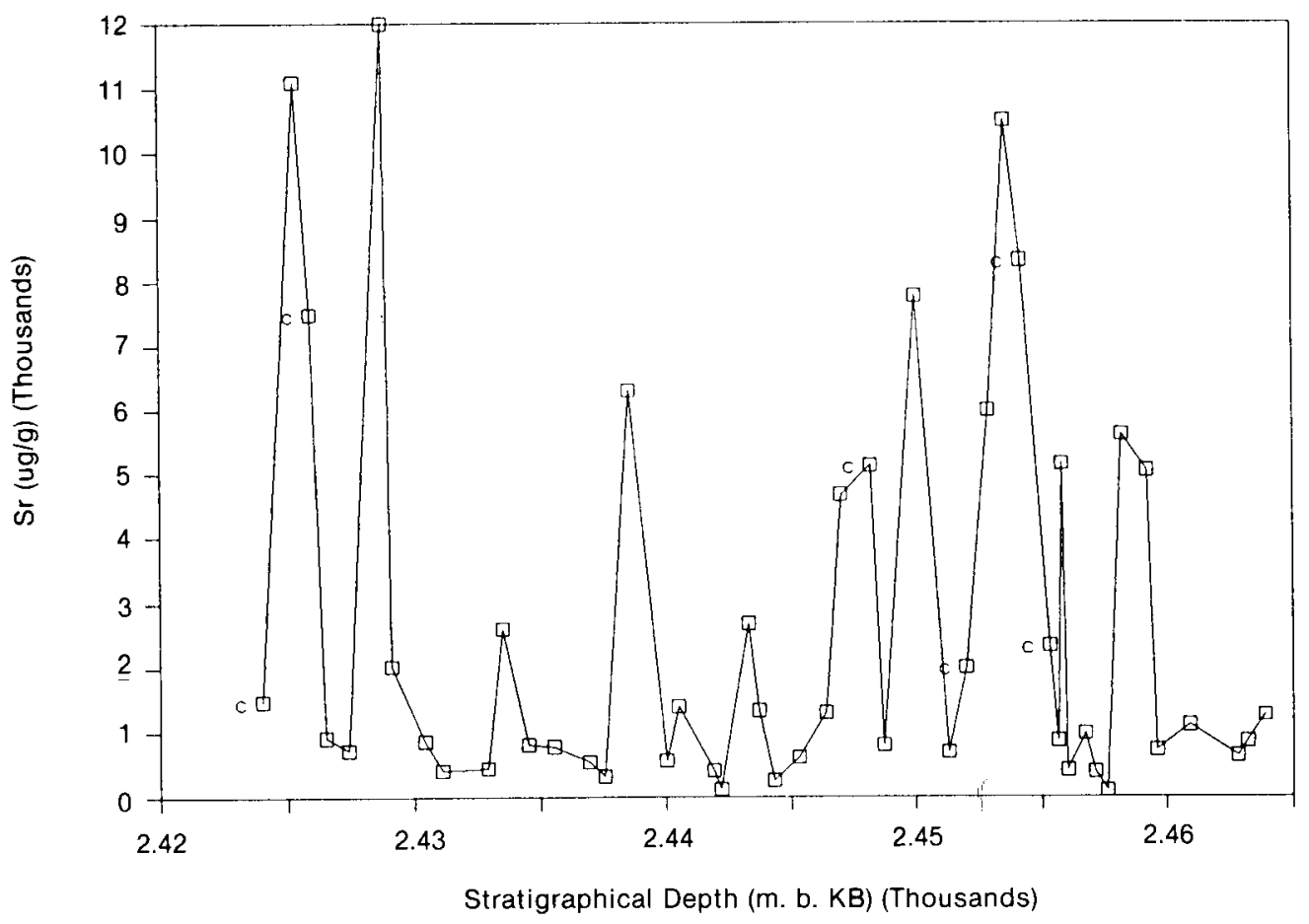

Fig. 8. Strontium determined by INAA (Table I) versus depth. The samples in which celestine has been encountered are marked with a " $c$ ". No correlation is seen between Sr and "c". 


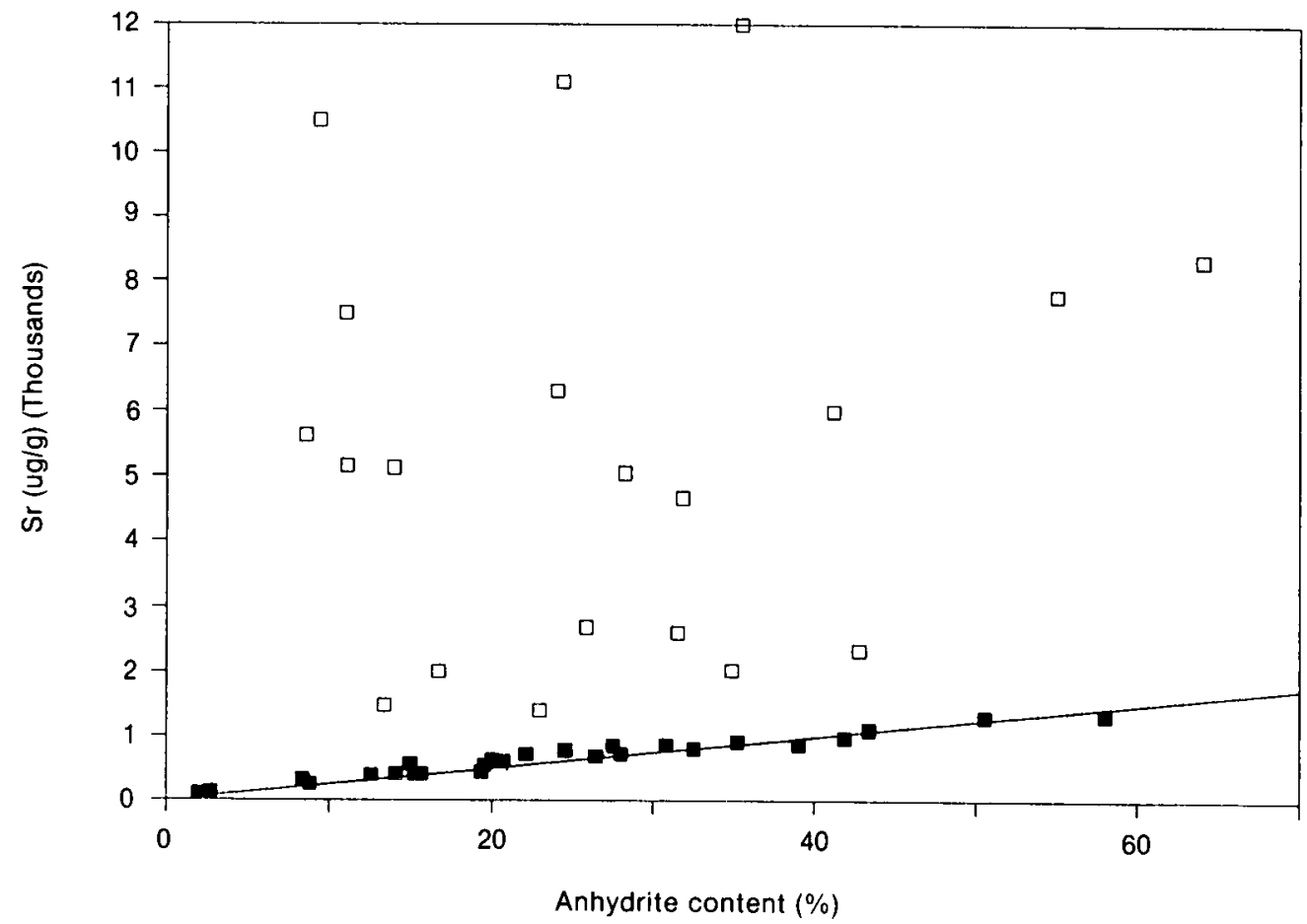

Fig. 9. Strontium determined by INAA (Table 1) versus anhydrite content (from atomic absorption, Table 4). 27 samples are termed the "low-Sr"-samples and are grouped along a straight line with a Srlanhydrite ratio between ca. 0.002 and ca. 0.004 (filled squares). The remaining samples have Srlanhydrite ratios greater than ca. 0.004 (up to ca. 0.1), and are termed the "high-Sr"-samples (open squares).

If the greater part of the $\mathrm{Sr}$ is present in anhydrite the content of anhydrite listed in Table 4 should correlate positively with $\mathrm{Sr}$ content given in Table 1 . In fact, some relationship is seen in the Sr-anhydrite cross plot (Figure 9). A total of 27 samples, or $56 \%$, are grouped along a straight line with a Sr/anhydrite ratio of ca. 0.003 . These 27 samples, which are termed the "low-Sr" samples, include both main facies types, A and $\mathrm{B}$. The remaining 20 samples - except for the rather anomalous sample number 48 from the lowermost part of the Ca-2 interval - have Sr/anhydrite ratios greater than ca. 0.003 (up to ca. 0.1). These we call the "high-Sr" samples. The sum of the amounts of dolomite and anhydrite is almost $100 \%$ in each sample (Table 4). Therefore it is not surprising that the Sr versus dolomite cross plot (Figure 10) is almost a reflected image of the $\mathrm{Sr}$ versus anhydrite cross plot. The same $56 \%$ of the samples clearly exhibit a negative correlation between $\mathrm{Sr}$ and dolomite content.

According to Veizer \& Demovic (1974) it is to be expected to find a somewhat higher $\mathrm{Sr} / \mathrm{Ca}$ ratio in the lagoonal facies (type $\mathrm{B}$ ) of sub-units I and III compared to the shoal facies (type A) of sub-unit II. This is not due to the chemistry of the primary facies, as the original coated grains of both facies A and B probably were built up of aragonite, similar to recent marine ooids/oncoids (Bathurst, 1976). However, during the dolomitization, which certainly occurred very early in the diagenetic history (Stentoft, 1990), the highly porous oolitic grainstone of facies A probably underwent more Sr-leaching than 


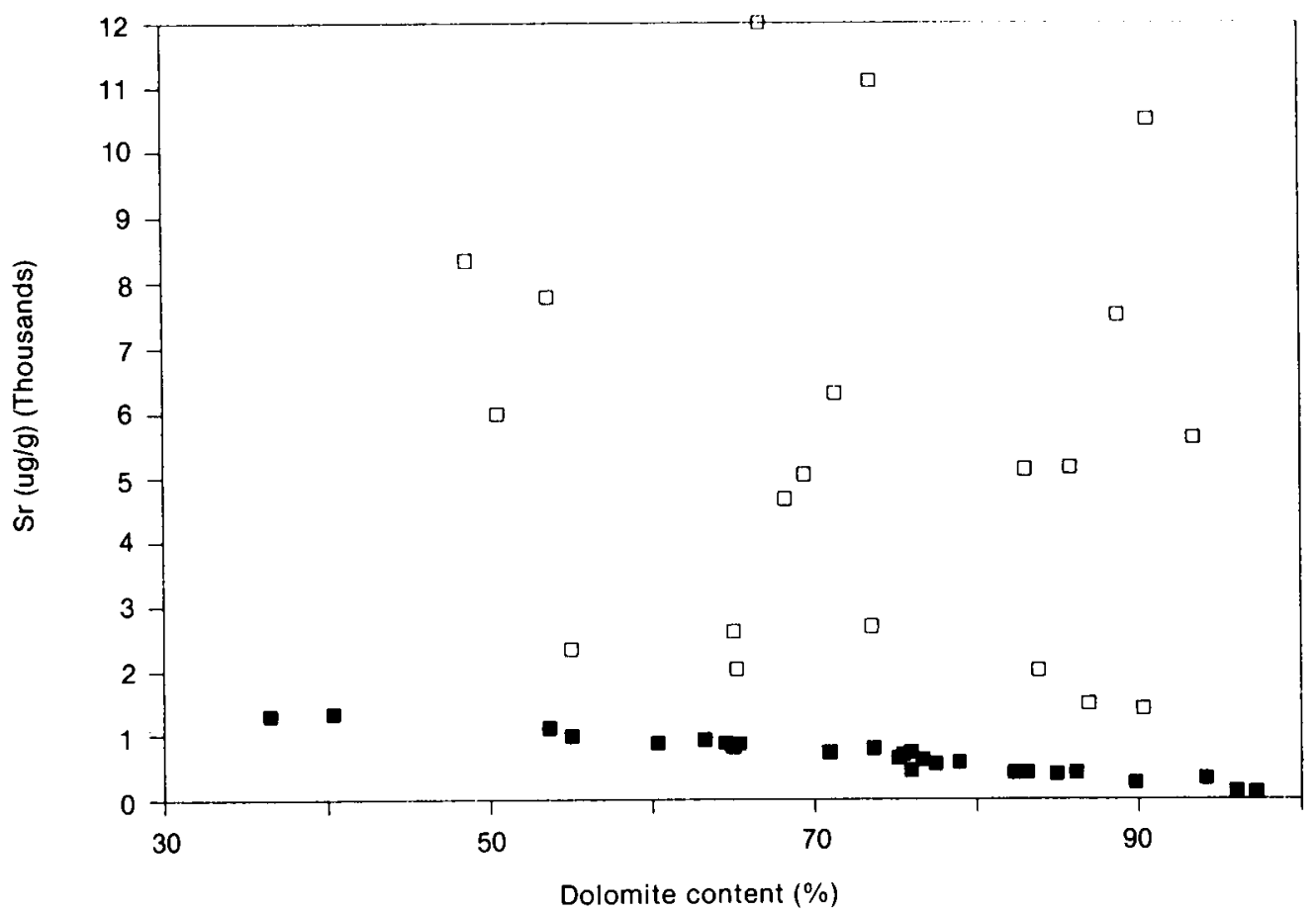

Fig. 10. Strontium determined by INAA (Table 1) versus dolomite content (from atomic absorption, Table 4). Note that this plot is almost an image of the $S r$ versus anhydrite cross plot (Figure 9): the same 27 low-Sr samples, which showed a positive Sr-anhydrite correlation, exhibit a negative correlation between Sr and dolomite content (filled squares).

the carbonate-mud-rich, low-permeable lagoonal sediments of facies B. As stated by Pingitore $(1982$; in connection with his discussion of the observations of Veizer \& Demovic, 1974) the presence of mud probably prevented an effective diffusion of $\mathrm{Sr}^{2+} \mathrm{du}-$ ring an initial aragonite/dolomite transformation, resulting in the production of a diagenetic dolomite high in $\mathrm{Sr}$. The $\mathrm{Sr} \times$ $1000 / \mathrm{Ca}$ ratios as well as the facies type are shown as a function of depth in Figure 11. It is evident from this plot that the $\mathrm{Sr} / \mathrm{Ca}$ ratio reaches high values only in the lagoonal carbonate facies (type $\mathrm{B}$ ). That this really reflects the $\mathrm{Sr} / \mathrm{Ca}$ ratios of the carbonates in an early diagenetic stage, and not mere $\mathrm{Sr}$ substitution for $\mathrm{Ca}$ in anhydrite, is supported by the lack of correlation between $\mathrm{Sr}$ and anhydrite content for the high-Sr samples (see Figure 9).
The density of anhydrite is $2.9-3.0 \mathrm{~g} / \mathrm{cm}^{3}$ whereas the density of dolomite is ca. 2.85 $\mathrm{g} / \mathrm{cm}^{3}$ (Phillipsborn, 1967). The density of the Ca-2 samples is increasing slightly with increasing anhydrite content only for the same 27 samples as noted above. So, if $\mathrm{Sr}$ was primarily bound in anhydrite, $\mathrm{Sr}$ would have to correlate positively with grain density. As can be seen in Figure 12 this is the case only for the 27 low-Sr samples. This further substantiates the above findings that the $\mathrm{Sr} / \mathrm{Ca}$ ratios do indeed reflect the original conditions.

The present porosity (and permeability) of the Ca-2 unit is highly controlled by the degree of anhydritization (Stentoft, 1990). It is therefore to be expected that the porosity is inversely proportional to the amount of anhydrite present. In Figure 13 is shown porosity versus Sr content, and a slight neg- 

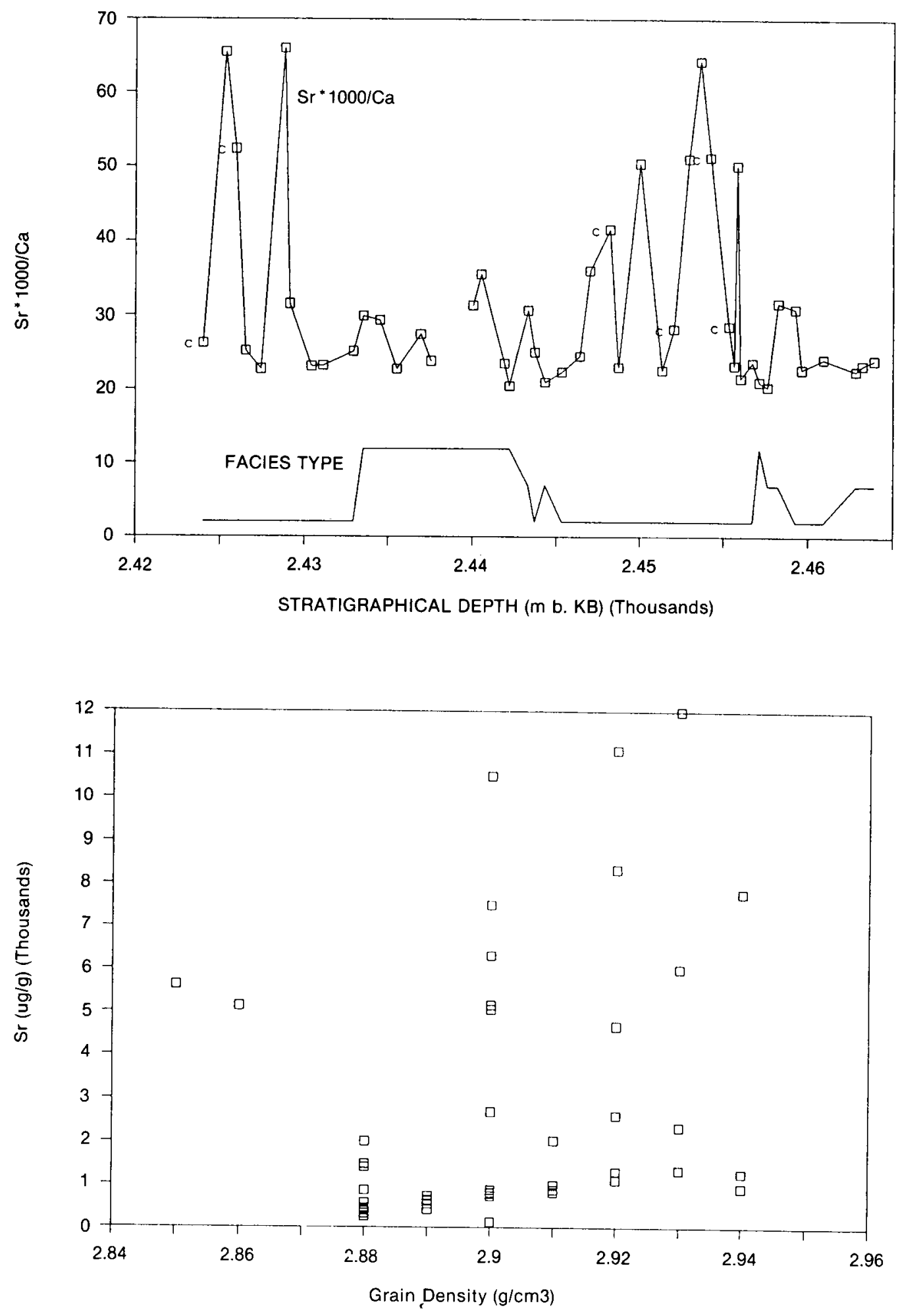
Fig. 11. Strontium * 1000/C a and facies type versus depth. The upper level of the facies type graph is the oolitic shoal facies (type A), the lower level is the lagoonal carbonates facies (type B), the intermediate points are the samples of indeterminate facies association. Note how most points of high Sr/Ca-ratio occur in the lagoonal facies.

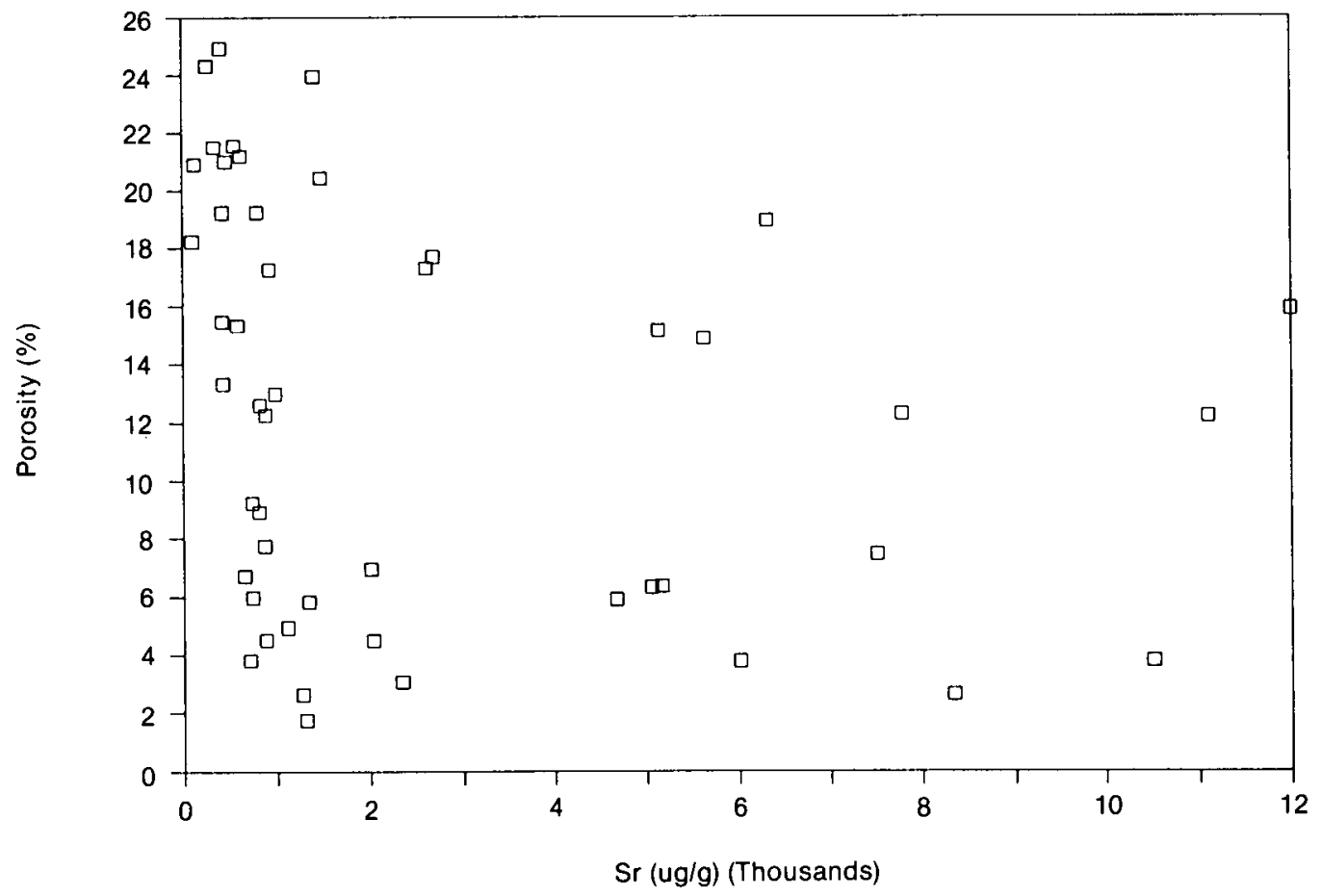

Fig. 13. Porosity (Table 6) versus Sr determined by INAA (Table 1). Note the negative correlation between the same 27 samples that showed a positive correlation between Sr and anhydrite content (Figure 9).

ative correlation is seen only between the low-Sr samples, i.e. the same 27 samples that showed a positive correlation between $\mathrm{Sr}$ and anhydrite content.

In summary we assert that the Sr-contents of the samples are related to at least two events in the diagenetic history of the $\mathrm{Ca}-2$ rocks: the early dolomitization of the original aragonite and the fairly late cementa- tion/replacement of anhydrite. In the low-Sr samples, which include a little more than half the samples, $\mathrm{Sr}$ is mainly bound to the late-diagenetic anhydrite. Here sulphate solutions may have percolated from the underlying Werra-sulfat (A-1), or it may stem from dissolved primary anhydrite/gypsum connected with the algal-rich areas of the rock or with areas of supratidal sabkha fa-

Fig. 12. Strontium determined by INAA (Table I) versus grain density (Table 6). Note that Sr is positively correlated with density for the low-Sr samples, the same 27 samples that showed a positive Sr-anhydrite correlation. 
cies (cf. Bathurst, 1976). Beside these low-Sr samples, dominated by anhydrite, the remaining samples, the high-Sr samples, are relatively rich in $\mathrm{Sr}$. The high-Sr samples mainly belong to the lagoonal, originally mud-rich, facies, and have preserved relatively large amounts of Sr from the dolomite, which precipitated during the initial aragonite/dolomite transformation.

\section{Dolomite}

According to Veizer (1983) trace elements occurring in the $\mu \mathrm{g} / \mathrm{g}$ concentration range and below are not suitable for studying carbonate sedimentation and diagenesis, as these elements are present mostly in the non-carbonate impurities of the $\mathrm{CaCO}_{3}$. Strontium, Fe, and $\mathrm{Zn}$, which may be present in dolomite replacing $\mathrm{Ca}$ and/or $\mathrm{Mg}$, are all found in concentrations above this limit in the samples of the $\mathrm{Ca}-2$ formation (cf. Table 1).

Strontium is, as we have seen, associated with anhydrite only for the low-Sr samples, for which reason facies variations of the $\mathrm{Sr} / \mathrm{Ca}$ ratios (e.g. lagoon versus bank environment; Veizer \& Demovic, 1974) can be demonstrated in the dolomite only for the high-Sr samples.

Barium may occur in small amounts in anhydrite and dolomite replacing $\mathrm{Ca}$ (Goldschmidt, 1954; Friedman, 1969; Fischer, 1972; Deer et al., 1976; Rao \& Naqvi, 1977). In celestine complete solid solution series between $\mathrm{BaSO}_{4}$ and $\mathrm{SrSO}_{4}$ are known to exist (Phillipsborn, 1967). Ba may also substitute for $\mathrm{K}$ in muscovite and other K-bearing minerals (Krauskopf, 1967).

Only about half of the INAA samples contained $\mathrm{Ba}$ in amounts above the detection limit: from $12 \mu \mathrm{g} / \mathrm{g}$ to $172 \mu \mathrm{g} / \mathrm{g}$ (Table

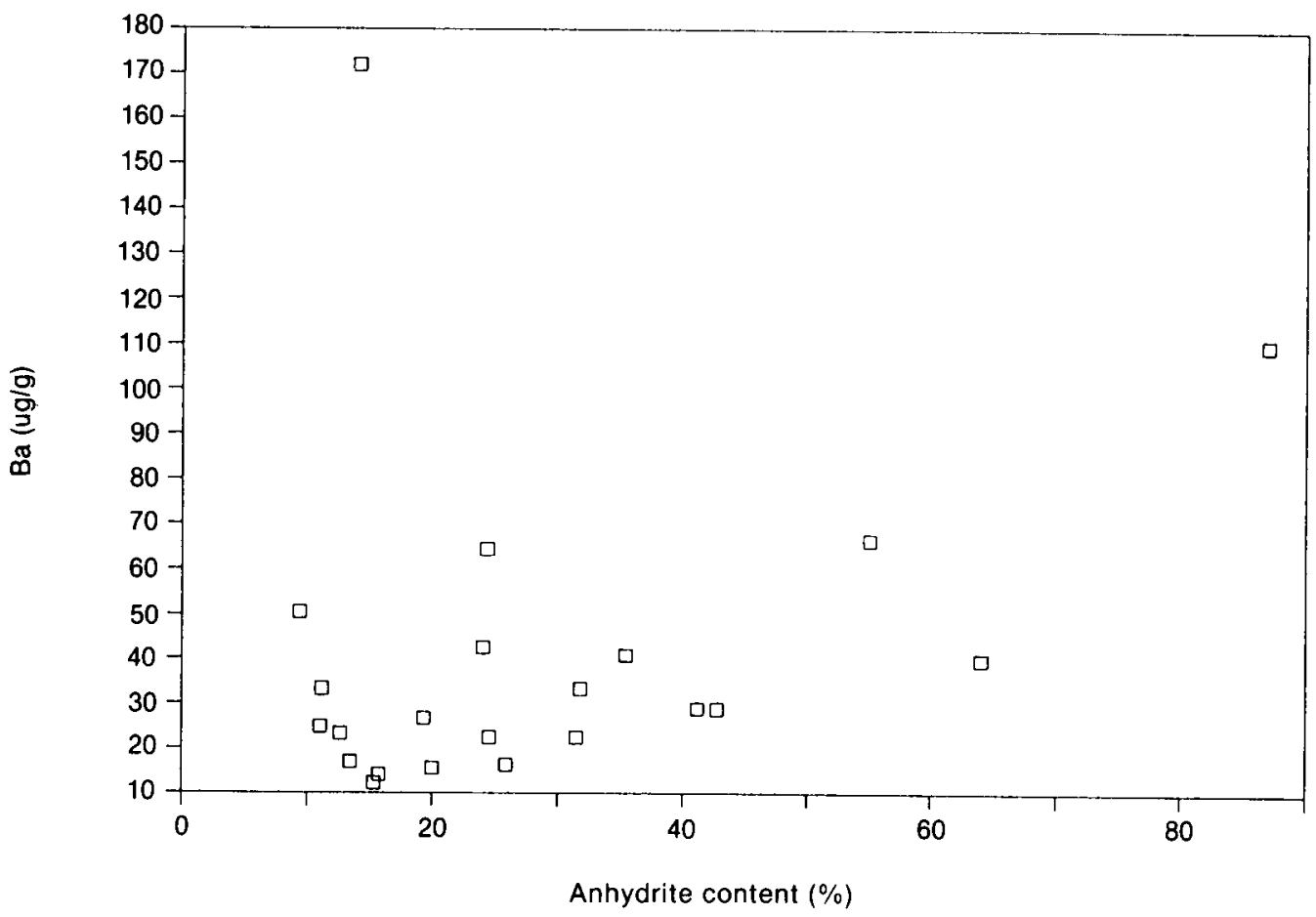

Fig. 14. Barium determined by INAA (Table 1) versus anhydrite content (Table 4). A marginal positive correlation is observed. 


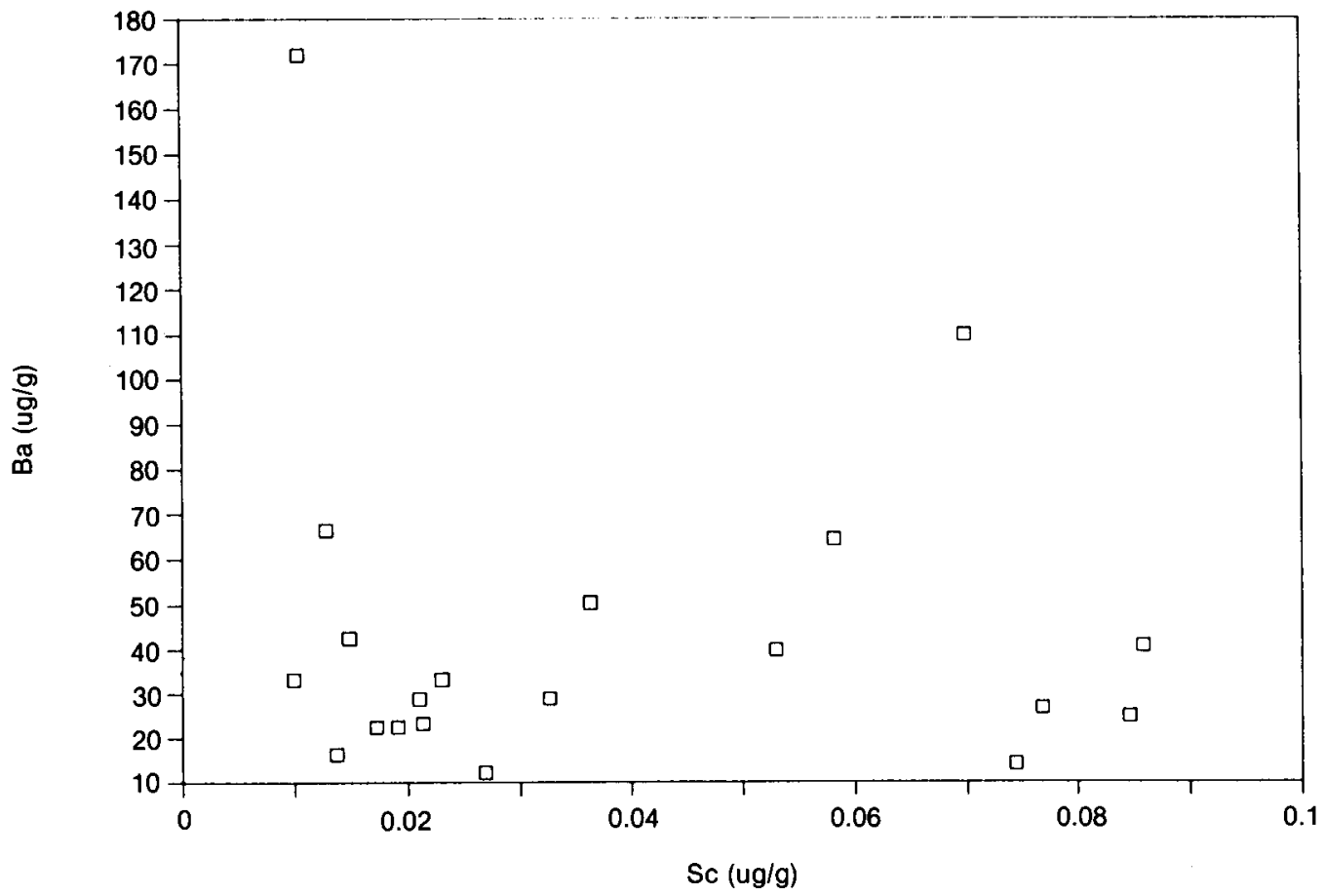

Fig. 15. Barium determined by INAA (Table 1) versus Sc determined by INAA (Table 1). No significant correlation is observed.

1). The relatively high concentration of $\mathrm{Ba}$ in two samples (sample no. 179 and 199) may possibly be due to contamination from the drilling mud, which has a somewhat, although not drastically, higher Ba content (ca. $450 \mu \mathrm{g} / \mathrm{g}$ ). We do not believe that contamination from the drilling mud is responsible for the bulk of the Ba abundances in the $\mathrm{Ca}-2$ samples, although it may play a minor role (see discussion of the drilling mud below).

If $\mathrm{Ba}$ primarily substituted for $\mathrm{Ca}$ in anhydrite, a positive correlation between $\mathrm{Ba}$ and anhydrite would be expected. There is, however, no unambiguous correlation between $\mathrm{Ba}$ and anhydrite content (Figure 14 ), so it is unlikely that $\mathrm{Ba}$ is situated primarily in the anhydrite.

Barium is probably not primarily bound in minerals of the EDTA-insoluble residue either, as no correlation is observed between $\mathrm{Ba}$ and the amount of undissolved (acid) residue (Table 4) or Sc (Figure 15).
In Figure $16 \mathrm{Ba}$ and facies type is shown as a function of stratigraphical depth, and it is seen that $\mathrm{Ba}$, like $\mathrm{Sr}$, reaches the higher values only in the intervals of lagoonal carbonates facies type B. It should be noted that many of the samples belonging to the oolitic shoal facies have missing $\mathrm{Ba}$ numbers, which means that the $\mathrm{Ba}$ contents in these samples are below the detection limit and thus quite low. It is thought, therefore, that the variation in $\mathrm{Ba}$ content reflects differences in the original (pre-diagenetic) environmental conditions, and that $\mathrm{Ba}$ is situated primarily in the dolomite.

Dolomite may contain small amounts of $\mathrm{Fe}^{2+}$ replacing $\mathrm{Mg}^{2+}$ (giving the rock a brownish tinge, Deer et al., 1976). However, no correlation is seen between $\mathrm{Mg}$ and $\mathrm{Fe}$, as is shown in Figure 17. On the other hand Fe correlates excellently with Sc (Figure 18), and REE, indicating a lithophile nature of this element. Insignificant amounts of pyrite $\left(\mathrm{FeS}_{2}\right)$ have been observed by optical mi- 

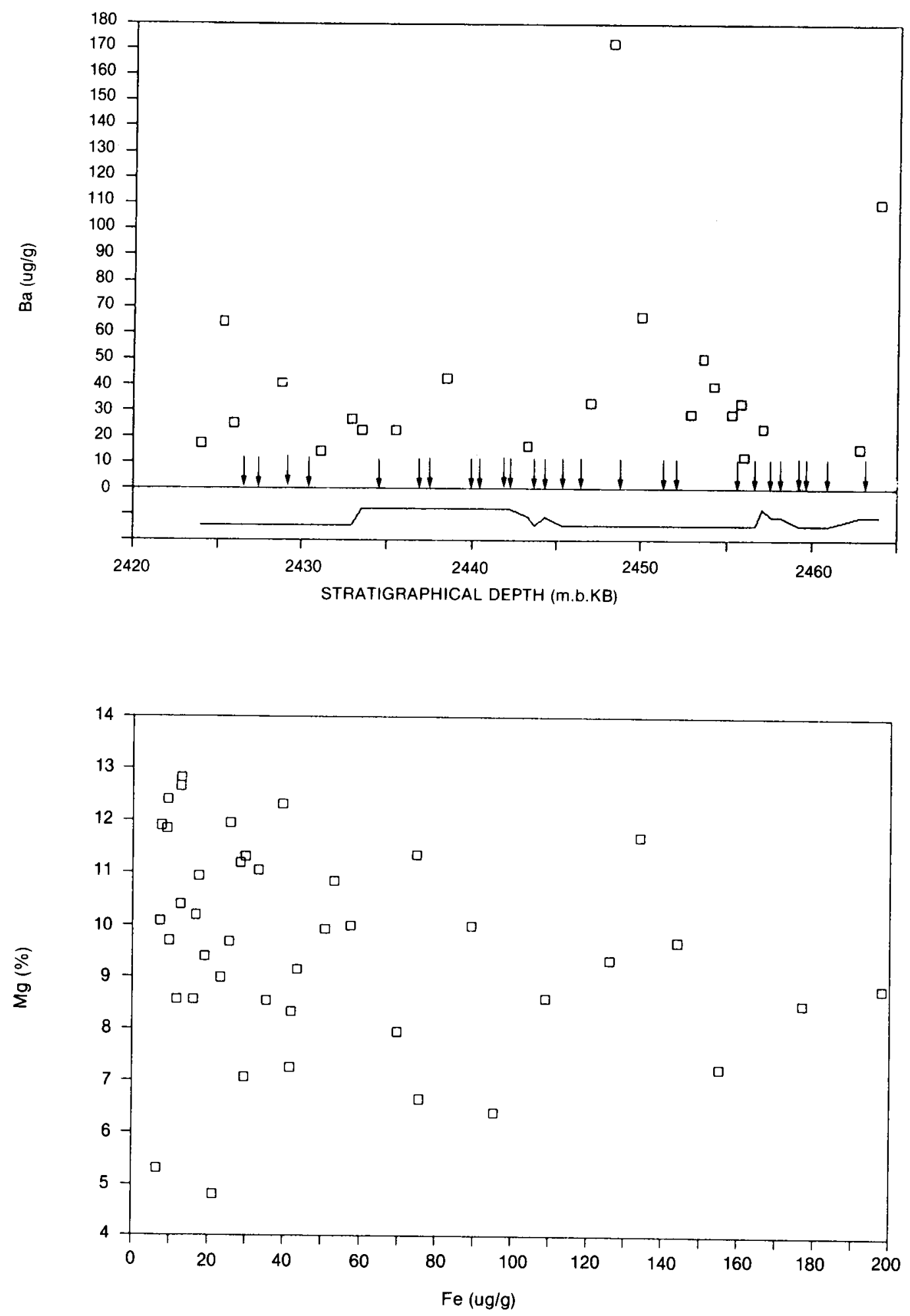
Fig. 16. Barium determined by INAA (Table 1) and facies type versus depth. Samples with Ba content below the detection limit are indicated with arrows. The upper level of the facies type graph is the oolitic shoal facies (type A), the lower level is the lagoonal carbonates facies (type B), the intermediate points are the samples of indeterminate facies association. Note how most points of high $\mathrm{Ba}$ occur in the lagoonal facies, quite the same picture as for Sr/Ca (Figure 11).

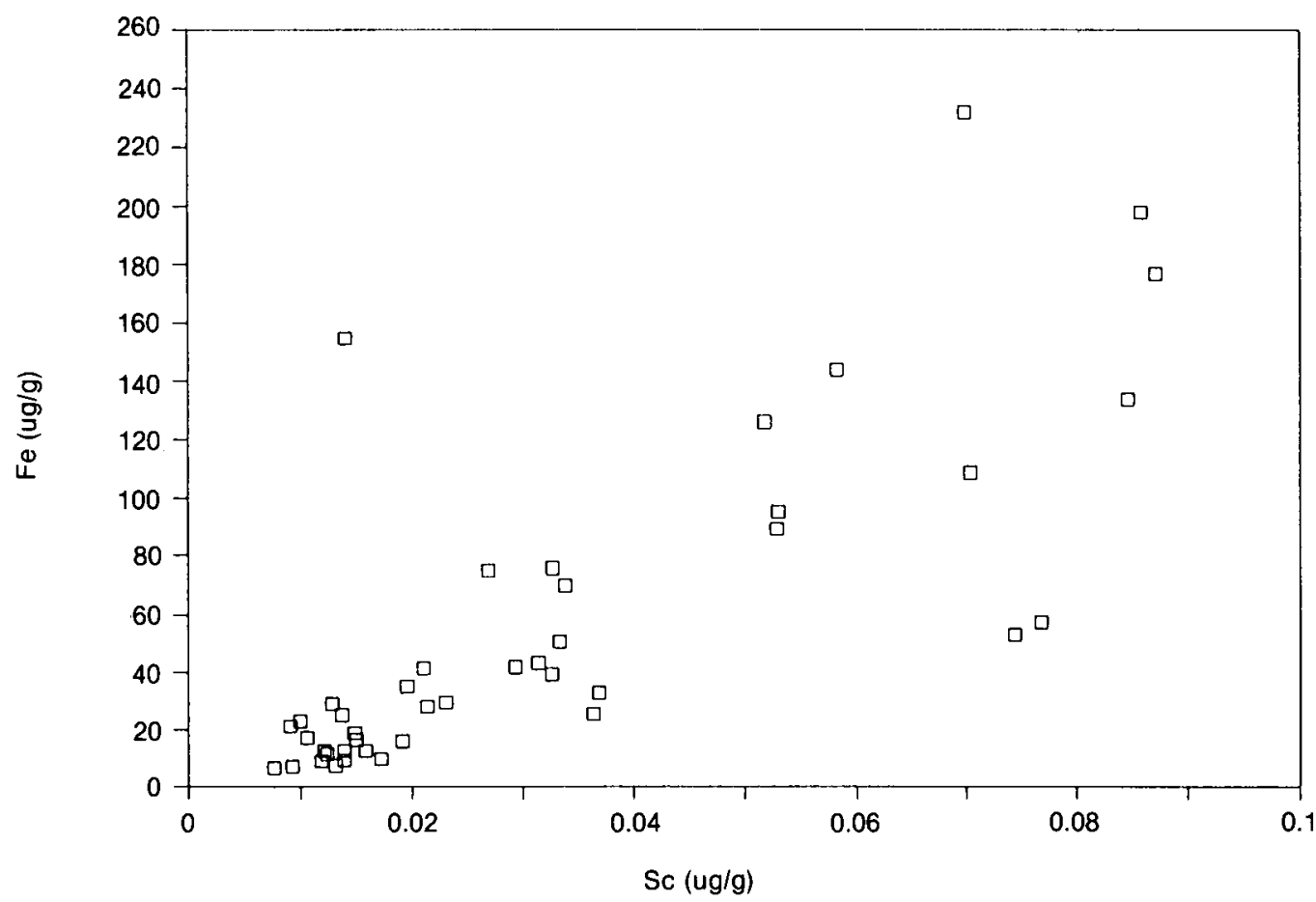

Fig. 18. Iron determined by INAA (Table 1) versus $S c$ also determined by INAA (Table 1). A strong positive correlation is observed, indicating a lithophile nature of iron in these rocks.

croscopy, and this mineral can consequently be ruled out as the major source for $\mathrm{Fe}$. The present results indicate that the $\mathrm{Fe}$ (or at least the majority of the $\mathrm{Fe}$ ) in some way is connected to clay minerals or other silicates, or possibly spinels.

Zinc, which has almost the same ionic radius as $\mathrm{Mg}$, has also previously been reported in dolomite (Deer et al., 1976; Botz $\&$ Müller, 1981). In the upper part of the Ca-1a interval of the Aabenraa- 1 well some
$\mathrm{Pb}-\mathrm{Zn}$ mineralization with galena ( $\mathrm{PbS})$, sphalerite $(\mathrm{ZnS})$, and other ore-minerals, was observed (Stentoft \& Nygaard, 1985), but none of these minerals have been noted neither in the core description nor in the thin sections of the Ca-2 interval in the L $\varnothing$ gumkloster-1 well. Like $\mathrm{Fe}, \mathrm{Zn}$ does not correlate with $\mathrm{Mg}$ (Figure 19), wherefore Zn probably do not primarily substitute for $\mathrm{Mg}$ in dolomite. We do not believe that $\mathrm{Zn}$ is situated primarily in ferromagnesian sil-

Fig. 17. Magnesium determined from atomic absorption (Table 4) versus Fe determined from INAA (Table 1). No significant correlation is observed. 


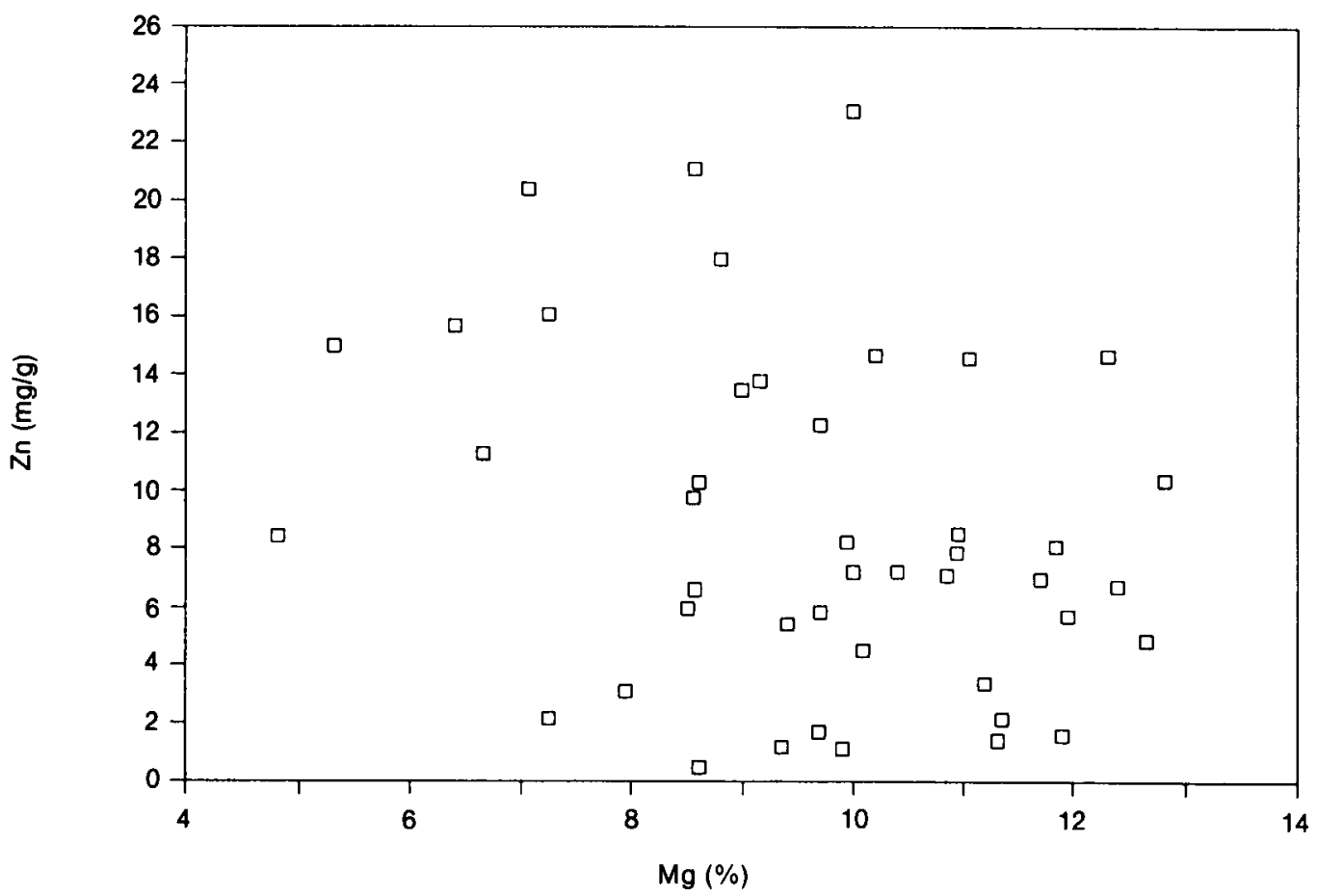

Fig. 19. Zinc determined by INAA (Table 1) versus $\mathrm{Mg}$ determined by atomic absorption (Table 4). No obvious correlation is observed.

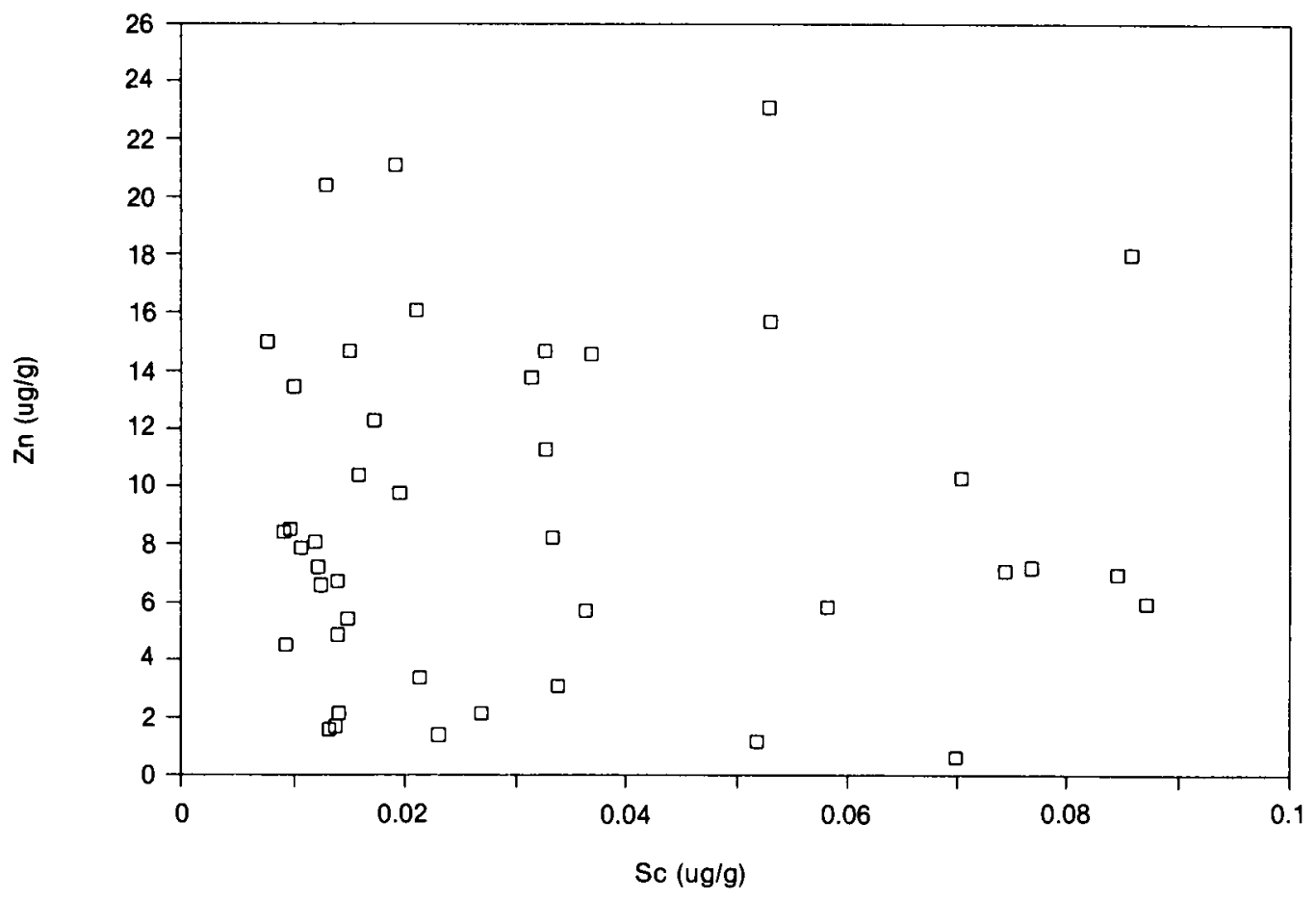

Fig. 20. Zinc determined by INAA (Table 1) versus Sc determined by INAA (Table 1). No obvious correlation is observed. 


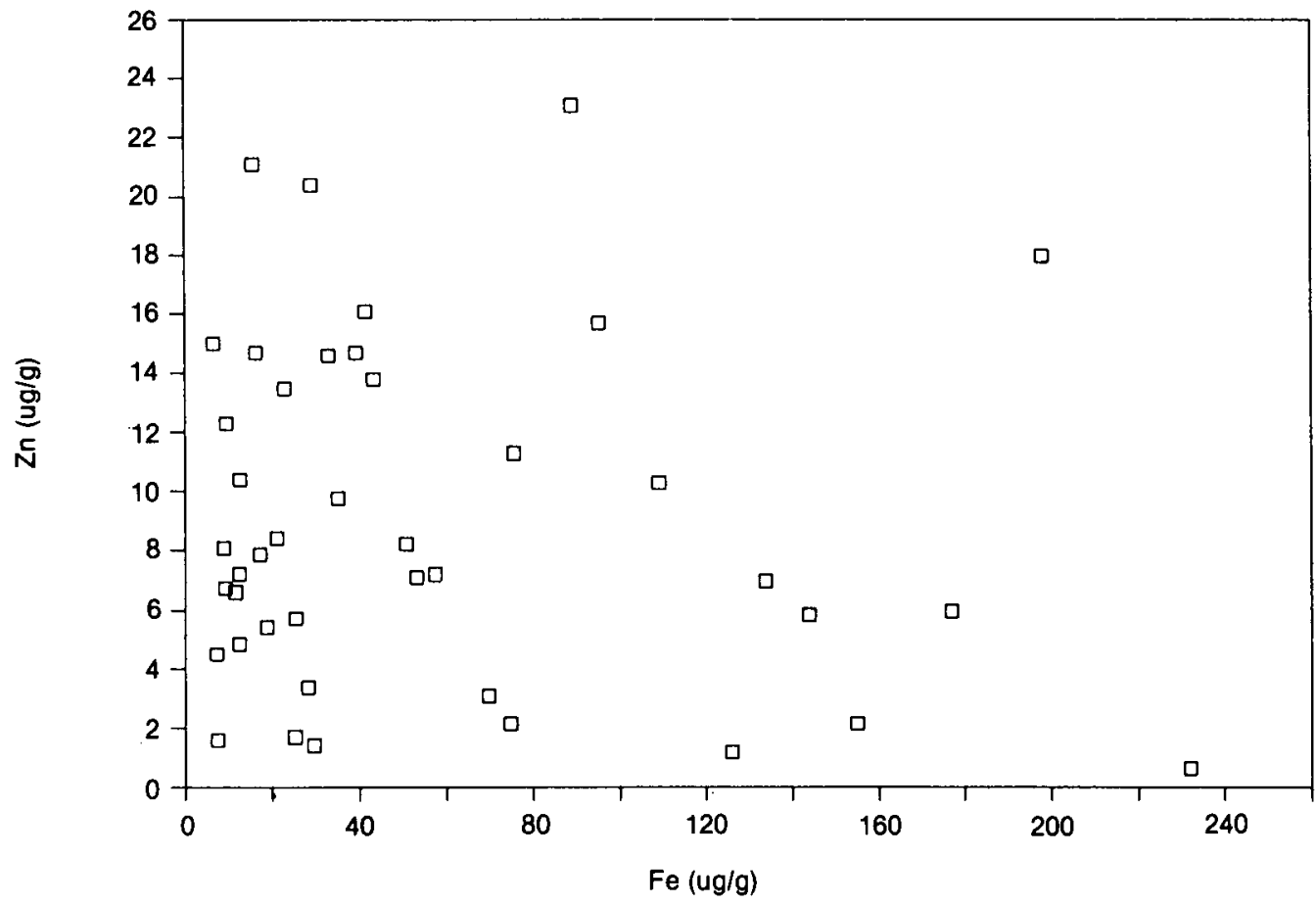

Fig. 21. Zinc determined by INAA (Table 1) versus Fe determined by INAA (Table 1). No obvious correlation is observed.

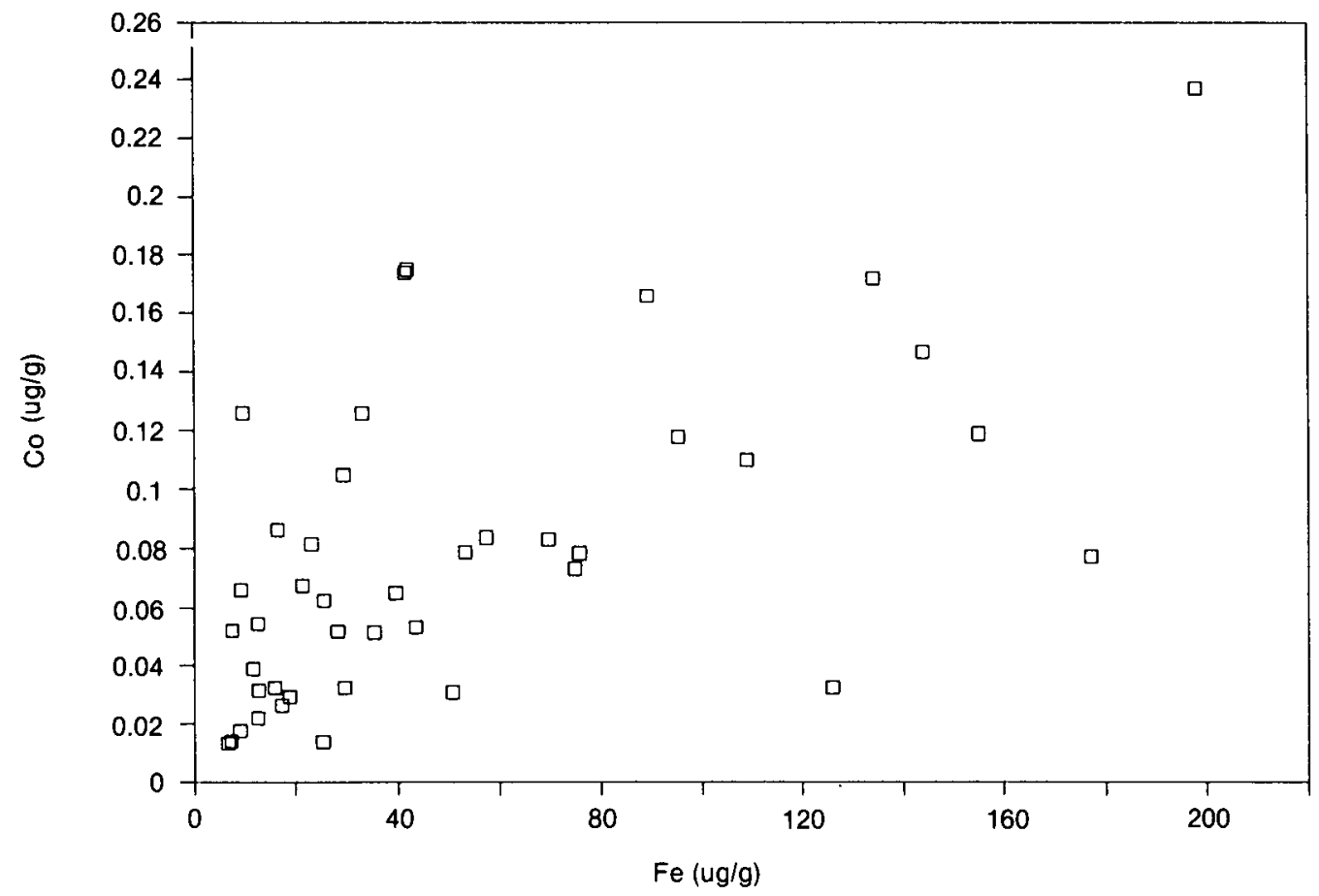

Fig. 22. Cobalt determined by INAA (Table 1) versus Fe determined by INAA (Table 1). A fair positive correlation is observed, indicating that $\mathrm{Co}$, like $\mathrm{Fe}$, probably is situated in the EDTAinsoluble residue, whether clay minerals, other silicates or spinels. 
icates either, as no correlation is observed between neither Sc nor Fe (Figures 20 and 21). We suspect that $\mathrm{Zn}$ may be present in organic substances, as organic rich shales are known to have relatively high $\mathrm{Zn}$ content (Siegel, 1979; Saban et al., 1984), or possibly as Smithsonite $\left(\mathrm{ZnCO}_{3}\right)$, but we have no firm evidence for these suspicions.

A positive correlation between $\mathrm{Co}$ and $\mathrm{Fe}$ was documented for shales and crystalline rocks by Carr \& Turekian (1961). Like Zn, some Co may also be adsorbed on clay minerals and ferric oxides (Krauskopf, 1956; Chester, 1965; Turekian, 1978). In the present data Co does not correlate with $\mathrm{Mg}$. Cobalt is, however, positively correlated with Fe, Sc, and REE (Figure 22). Cobalt therefore, like $\mathrm{Fe}$, is probably for a great part connected to the EDTA-insoluble residue of the $\mathrm{Ca}-2$ dolomite, whether clay minerals, other silicates or spinels, where Co may substitute for Fe in octahedral sites.
Contrary to $\mathrm{Zn}$, Co is not enriched in black shales relative to normal shales, indicating that a Co accumulation is generally not taking place in bituminous matter (Vine et al., 1970). The Co content of crude oil is mostly far less than the Co content of shales (Shah et al., 1970), although Co has been reported to occur in asphaltenes (Jacobs et al., 1984).

Other elements occasionally of chalcophile nature were detected in this study: $\mathrm{Cr}$, As, Se, and Hf, but it is thought that these elements are associated with the EDTA-insoluble residue and will thus be discussed under this phase.

\section{The salt phases}

The distribution of $\mathrm{Na}, \mathrm{Br}, \mathrm{K}, \mathrm{Rb}$, and $\mathrm{Cs}$ are shown on Figure 23, and it is seen that $\mathrm{Na}$ and $\mathrm{Br}$ form one co-varying group, and $\mathrm{K}, \mathrm{Rb}$, and $\mathrm{Cs}$ another distinctly different co-varying group.

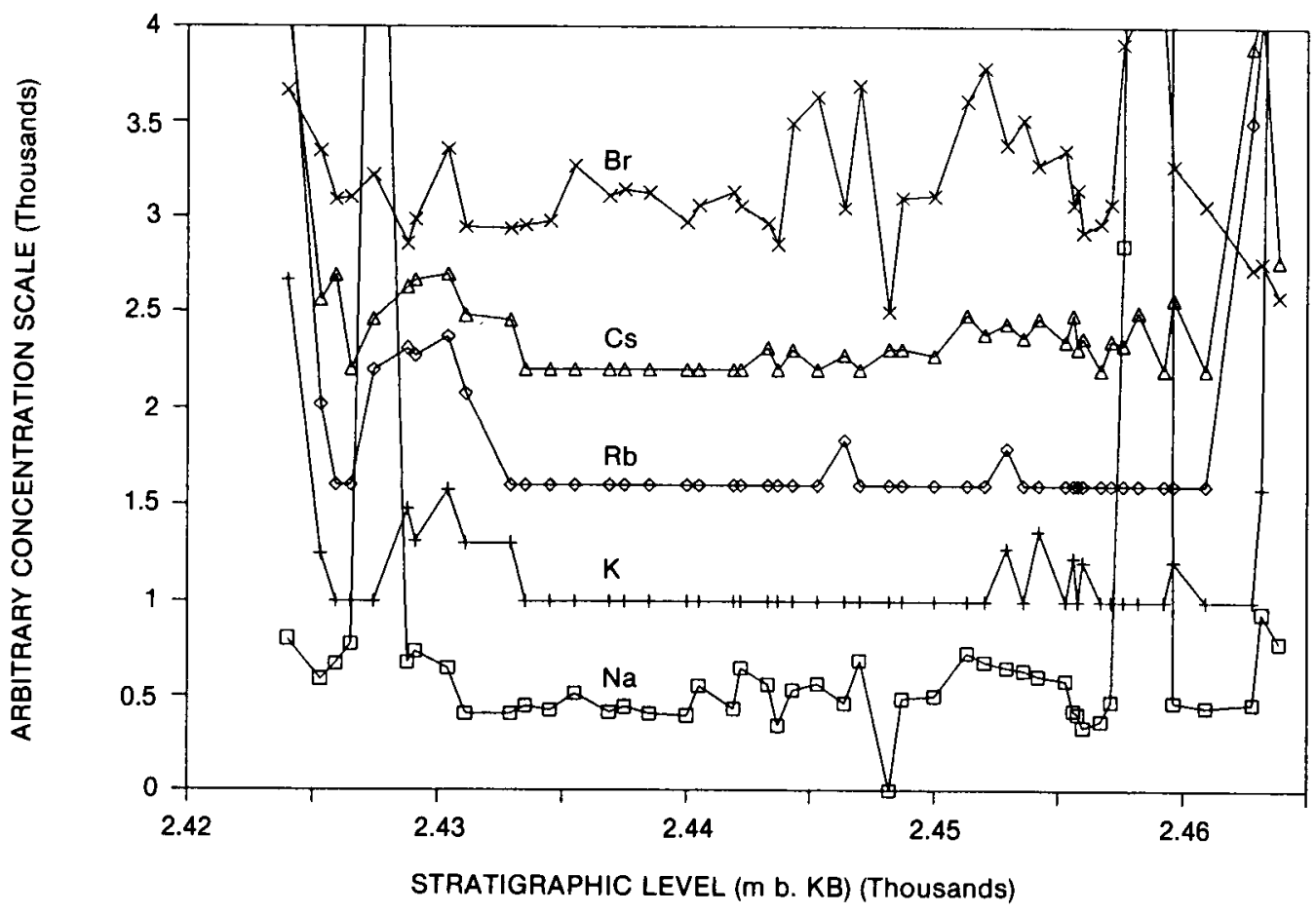

Fig. 23. Sodium, $\mathrm{Br}, \mathrm{K}, \mathrm{Rb}$, and $\mathrm{Cs}$ determined by INAA (Table 1) versus depth. It is seen that $\mathrm{Na}$ and $B r$ form one co-varying group, and $K, R b$, and $C s$ another distinctly different co-varying group. 
Sodium and $\mathrm{Br}$ are probably situated in halite ( $\mathrm{NaCl}$ ) (Fuge, 1974), which is observed by optical microscopical examination of thin sections to occur rather frequently. No correlation is observed between $\mathrm{Na}$ and $\mathrm{Ca}$, so it is unlikely that $\mathrm{Na}$ substitute for $\mathrm{Ca}$ in either dolomite or anhydrite, which also seems unlikely for anhydrite in the light of the results of Kushnir (1980). It is interesting to note that due to the dissimilarity of the variational pattern of the $\mathrm{Na}-\mathrm{Br}$-group and the K-Rb-Cs-group, $\mathrm{K}, \mathrm{Rb}$, and $\mathrm{Cs}$ must be situated in a mineral different from halite. As no other evaporite minerals were observed to occur frequently, this suggests that $\mathrm{K}, \mathrm{Rb}$, and $\mathrm{Cs}$ are situated mainly in the clay minerals or K-feldspars (Heier \& Billings, 1970). As clay minerals are by far the most abundant of these, we find it most likely that $\mathrm{K}, \mathrm{Rb}$, and $\mathrm{Cs}$ occur mainly in clay minerals. This is not out of line with the abundance pattern of the REE, mostly La, $\mathrm{Yb}$, and $\mathrm{Lu}$, although no strong correlation exists between the $\mathrm{K}-\mathrm{Rb}-\mathrm{Cs}$-group and the REE.

\section{The EDTA-insoluble phases}

X-ray diffraction carried out on the EDTAinsoluble residue showed only three phases. Firstly a muscovite or clay mineral. Secondly, some quartz, and thirdly an X-ray amorphous Al-silicate. The abundances of the two latter phases are about the same order of magnitude as the clay minerals (Stentoft et al. 1990). From optical inspection it is clear that the quartz grains are very regular and sometimes form coating on other mineral grains, and are therefore thought to be of secondary diagenetic origin as discussed by Stentoft (1990).

\section{Rare Earth Elements, Sc, and Th}

Carbonaceous chondrites of type CI constitute the material most resembling the solar composition of which the Earth was once made (Wasson, 1985). As is customary CI abundances of REE have been used as a normalizing standard. The normalized abundances of the average composition of the $\mathrm{Ca}-2$ rocks are shown in Figure 24a. Superimposed on Figure $24 \mathrm{a}$ is shown the average REE composition of the two main facies types. On Figure $24 b$ is shown the North American Shale Composite, NASC, (Haskin et al., 1968) and average seawater abundances (Mason, 1966; Krauskopf, 1967). It is evident from Figure 24a that there is a pronounced enrichment in LREE (Light REE: La-Sm) relative to HREE (Heavy REE: Gd-Lu). The composite numbers LREE and HREE and their ratio, LREE/HREE are listed in Table 8.

The CI-normalized abundances for individual samples generally show a negative Eu-anomaly, a fact also reflected in the overall Ca-2 average shown in Figure 24a. This is characteristic also for the NASC and the average seawater (Figure 24b), and shows that the REE-bearing minerals in the $\mathrm{Ca}-2$ unit could be related to either of these materials, it does not allow us to discriminate between them. Other workers similarly found that formation waters produced from Gulf Coast Basin oil fields exhibited REEpatterns almost indistinguishable from NASC (Macpherson et al., 1988).

The $\mathrm{CI}$ and $\mathrm{Sm}$ normalized abundances of seawater show a pronounced negative Ceanomaly (Figure 24b), which is characteristic for the seawater abundances as opposed to the NASC abundances. The conventional explanation for the negative $\mathrm{Ce}$ anomaly in seawater is that the residence time for $\mathrm{Ce}$ in the ocean is ca. 4 times lower (Mason, 1966) than for the rest of the REE. When leaving the seawater traditional wisdom says that $\mathrm{Ce}$ gets bound in fish teeth, fish scales, tests, and manganese nodules, if such are present (Fleet, 1984; Palmer, 1985; Haynes etal., 1985; Oudin \& Cocherie, 1988). In the $\mathrm{Ca}-2$ unit we have not, however, observed any manganese nodules or fish teeth, so this source can probably be ruled out in connection with the present rocks. It has 
A

A

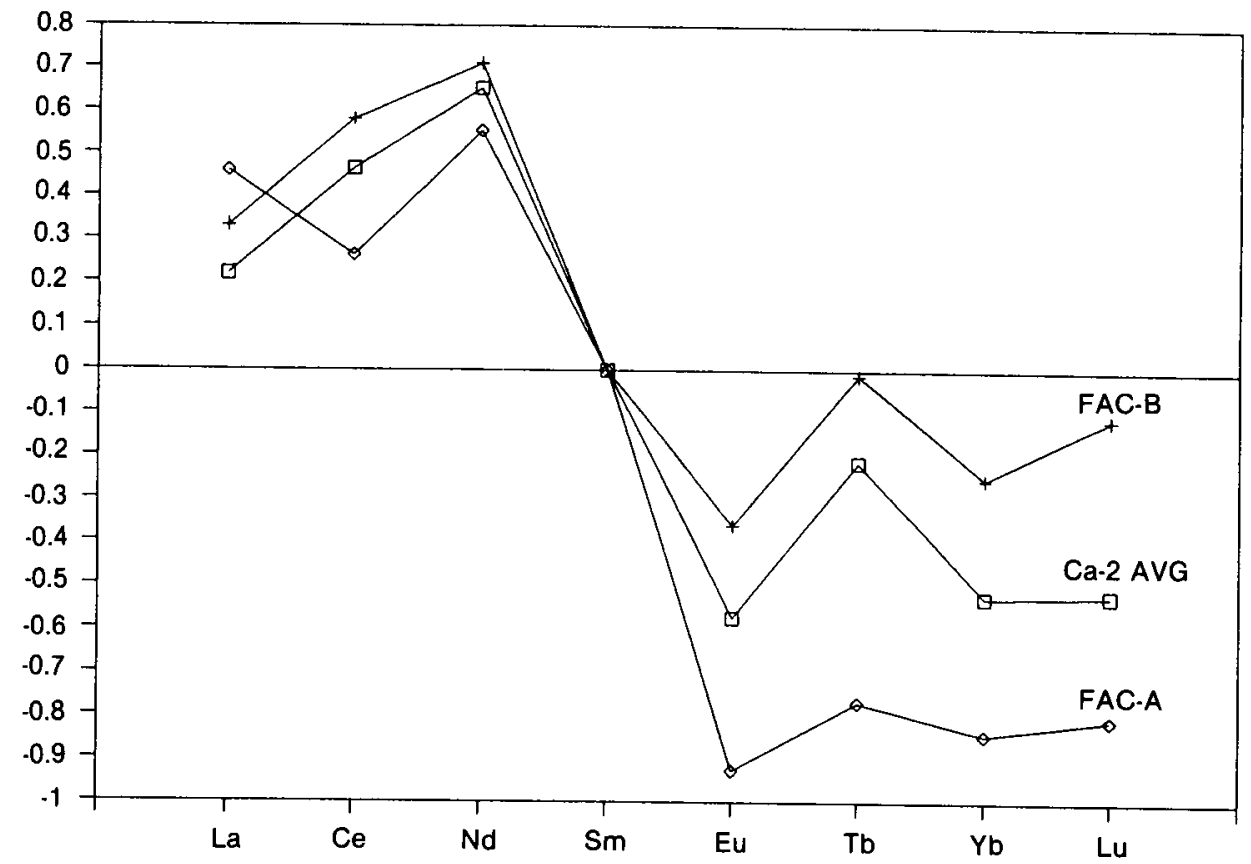

$\mathbf{B}$

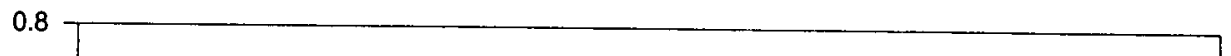

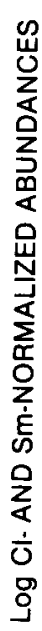

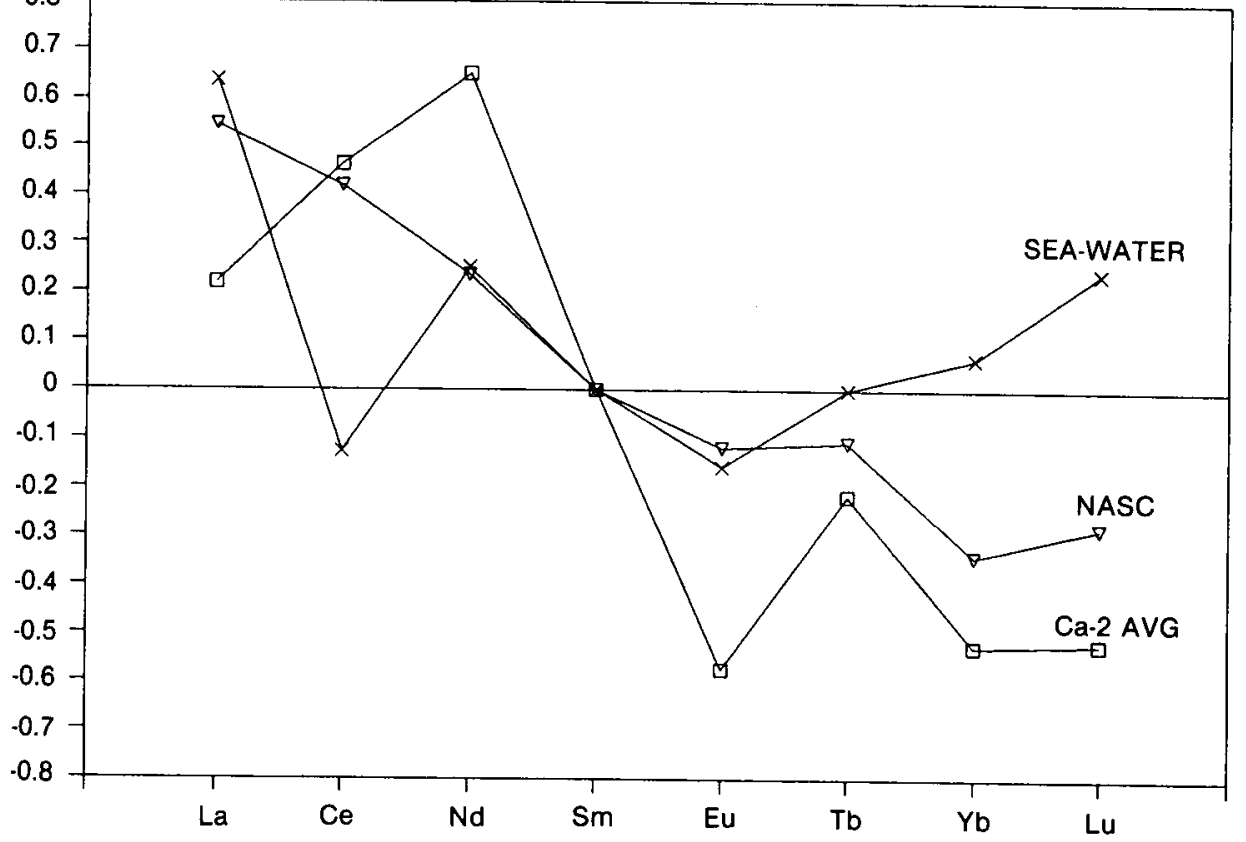

Fig. 24. A: REE abundances normalized to CI abundances and Sm (Wasson, 1985): Average of all $C a-2$ samples analyzed. Average of all samples belonging to the lagoonal facies type $(B)$. Average of all samples belonging to the oolitic shoal facies type $(A)$. Note the negative Ce-anomali in the oolitic shoal facies $(A)$. The uncertainty in the average Ce abundances of the two facies is $60 \%$ for facies type $B$ and $40 \%$ for facies type $A$ (one standard deviation). B: REE abundances normalized to $C I$ abundances and Sm (Wasson, 1985): Average of all Ca-2 samples analyzed. North American Shale Composite, NASC, (Haskin et al., 1968). Average seawater abundances (Mason, 1966; Krauskopf, 1967). 


\begin{tabular}{|c|c|c|c|c|c|c|}
\hline $\begin{array}{l}\text { Depth } \\
\text { m }\end{array}$ & Name & $\begin{array}{c}\text { REE-av } \\
\mu \mathrm{g} / \mathrm{g}\end{array}$ & $\begin{array}{c}\text { LREE } \\
\mu \mathrm{g} / \mathrm{g}\end{array}$ & $\begin{array}{c}\mathrm{HREE} \\
\mu \mathrm{g} / \mathrm{g}\end{array}$ & $\begin{array}{l}\text { LREE/ } \\
\text { HREE }\end{array}$ & $\begin{array}{l}\text { Facies } \\
\text { Type }\end{array}$ \\
\hline 2424.0 & -132 & 0.951 & 1.630 & 0.0455 & 36 & B \\
\hline 2425.3 & -129 & 0.451 & 0.876 & 0.0278 & 32 & B \\
\hline 2425.9 & -127 & 0.479 & 0.807 & 0.0512 & 16 & B \\
\hline 2426.5 & -124 & 0.270 & 0.527 & 0.0145 & 36 & B \\
\hline 2427.4 & -123 & 0.421 & 0.949 & 0.0266 & 36 & B \\
\hline 2428.8 & -116 & 0.621 & 0.825 & & & B \\
\hline 2429.1 & -118 & 0.379 & 0.622 & 0.0182 & 34 & B \\
\hline 2430.4 & -112 & 0.371 & 0.725 & 0.0200 & 36 & B \\
\hline 2431.1 & -110 & 0.547 & 0.905 & 0.0138 & 66 & B \\
\hline 2432.9 & -108 & 0.624 & 1.032 & 0.0134 & 77 & B \\
\hline 2433.5 & -106 & 0.348 & 0.518 & 0.0117 & 44 & A \\
\hline 2434.5 & -104 & 0.539 & 0.892 & 0.0142 & 63 & A \\
\hline 2435.5 & -101 & 0.486 & 0.803 & 0.0134 & 60 & A \\
\hline 2436.9 & -99 & 0.418 & 0.829 & 0.0091 & 91 & A \\
\hline 2437.5 & -98 & 0.345 & 0.681 & 0.0112 & 61 & A \\
\hline 2438.5 & -96 & 0.537 & 0.934 & 0.0094 & 99 & A \\
\hline 2440.0 & -93 & 0.360 & 0.594 & 0.0147 & 40 & A \\
\hline 2440.5 & -91 & 0.394 & 0.781 & 0.0084 & 93 & A \\
\hline 2441.9 & -88 & 0.329 & 0.650 & 0.0078 & 83 & A \\
\hline 2442.2 & -197 & 0.317 & 0.317 & & & A \\
\hline 2443.3 & -191 & 0.220 & 0.435 & 0.0045 & 98 & $\mathrm{~A} / \mathrm{B}$ ? \\
\hline 2443.7 & -188 & 0.214 & 0.285 & & & B \\
\hline 2444.3 & -190 & 0.277 & 0.458 & 0.0052 & 87 & $\mathrm{~A} / \mathrm{B}$ ? \\
\hline 2445.3 & -183 & 0.431 & 0.574 & & & B \\
\hline 2446.4 & -182 & 0.148 & 0.196 & & & B \\
\hline 2447.0 & -180 & 0.110 & 0.215 & 0.0068 & 31 & B \\
\hline 2448.2 & -179 & 0.386 & 0.769 & 0.0032 & 240 & B \\
\hline 2448.7 & -175 & 0.212 & 0.282 & & & B \\
\hline 2450.0 & -171 & 0.140 & 0.186 & & & B \\
\hline 2451.3 & -168 & 0.386 & 0.512 & & & B \\
\hline 2452.0 & -165 & 0.287 & 0.380 & & & B \\
\hline 2452.9 & -163 & 0.274 & 0.452 & 0.0048 & 95 & B \\
\hline 2453.6 & -162 & 0.329 & 0.437 & & & B \\
\hline 2454.2 & -160 & 0.400 & 0.531 & & & B \\
\hline 2455.3 & -155 & 0.304 & 0.504 & 0.0046 & 110 & B \\
\hline 2455.6 & -157 & 0.351 & 0.575 & 0.0235 & 24 & B \\
\hline 2455.8 & -149 & 0.254 & 0.497 & 0.0125 & 40 & B \\
\hline 2456.0 & -151 & 0.606 & 0.806 & & & B \\
\hline 2456.7 & -144 & 0.426 & 0.567 & & & B \\
\hline 2457.1 & -146 & 0.451 & 0.897 & 0.0042 & 215 & A \\
\hline 2457.6 & -143 & 0.319 & 0.631 & 0.0089 & 71 & $\mathrm{~A} / \mathrm{B}$ ? \\
\hline 2458.2 & -138 & 1.054 & 1.575 & & & $\mathrm{~A} / \mathrm{B}$ ? \\
\hline 2459.2 & -135 & 0.890 & 1.330 & & & B \\
\hline 2459.6 & -134 & 0.486 & 0.646 & & & B \\
\hline 2460.9 & -204 & 0.451 & 0.451 & & & B \\
\hline 2462.8 & -200 & 0.898 & 1.334 & 0.0222 & 60 & $\mathrm{~A} / \mathrm{B}$ ? \\
\hline 2463.2 & -201 & 0.777 & 1.152 & 0.0249 & 46 & $\mathrm{~A} / \mathrm{B} ?$ \\
\hline 2463.9 & -199 & 1.131 & 2.101 & 0.2368 & 9 & $\mathrm{~A} / \mathrm{B}$ ? \\
\hline
\end{tabular}

Table 8. Average REE, Light REE (LREE = La-Sm), Heavy REE (HREE = Gd-Lu), and facies type (from Table 6). Facies type A: an oolitic shoal facies. Facies type B: a lagoonal carbonate facies. 
been reported that modern marine carbonates often have pronounced negative $\mathrm{Ce}$ anomalies (Shaw \& Wasserburg, 1985; Palmer, 1985; Banner et al., 1988), whereas older carbonates often lack a significant $\mathrm{Ce}$ anomaly (Jarvis et al., 1975; Parekh et al., 1977; Graf, 1984). Our data conform to this general picture, being relatively old and exhibiting only a slight, if any, negative $\mathrm{Ce}$ anomaly. It is, however, evident from Figure 24a that facies type $A$, the oolitic shoal facies, exhibit a negative $\mathrm{Ce}$ anomaly, whereas type $\mathrm{B}$, the lagoonal carbonate facies, do not. This is interpreted as reflecting a pronounced seawater input and mixing at the time of deposition of the oolitic shoal facies. Likewise the deposition of the lagoonal carbonate facies is interpreted to have taken place in an environment where the mixing with sea water was less intense, and where the large amounts of carbonate mud present to some degree prevented contact between sediment and sea water that occassionally spilled over the barrier.

The negative $\mathrm{Ce}$ anomaly for the oolitic shoal facies is, however, only small, and the question remains where the bulk of the REEs are situated, and what their original source was. Trivalent REEs, predominantly $\mathrm{Ce}$, are known to substitute for $\mathrm{Ca}$ in apatite (Deer et al., 1976; Felsche, 1978). In the Ca-2 interval we have, however, not observed any occurrence of apatite, neither by $\mathrm{X}$-ray diffraction nor by optical microscopy, and consequently this mineral has been ruled out as a major REE bearing phase.

A fair correlation between lithophile elements, such as Sc, Th, and Fe, and REE is observed at the extremes of the present interval (see Figure 25). We interpret this fact as indicating that the bulk of the REE is bound to the EDTA-insoluble minerals, e.g.

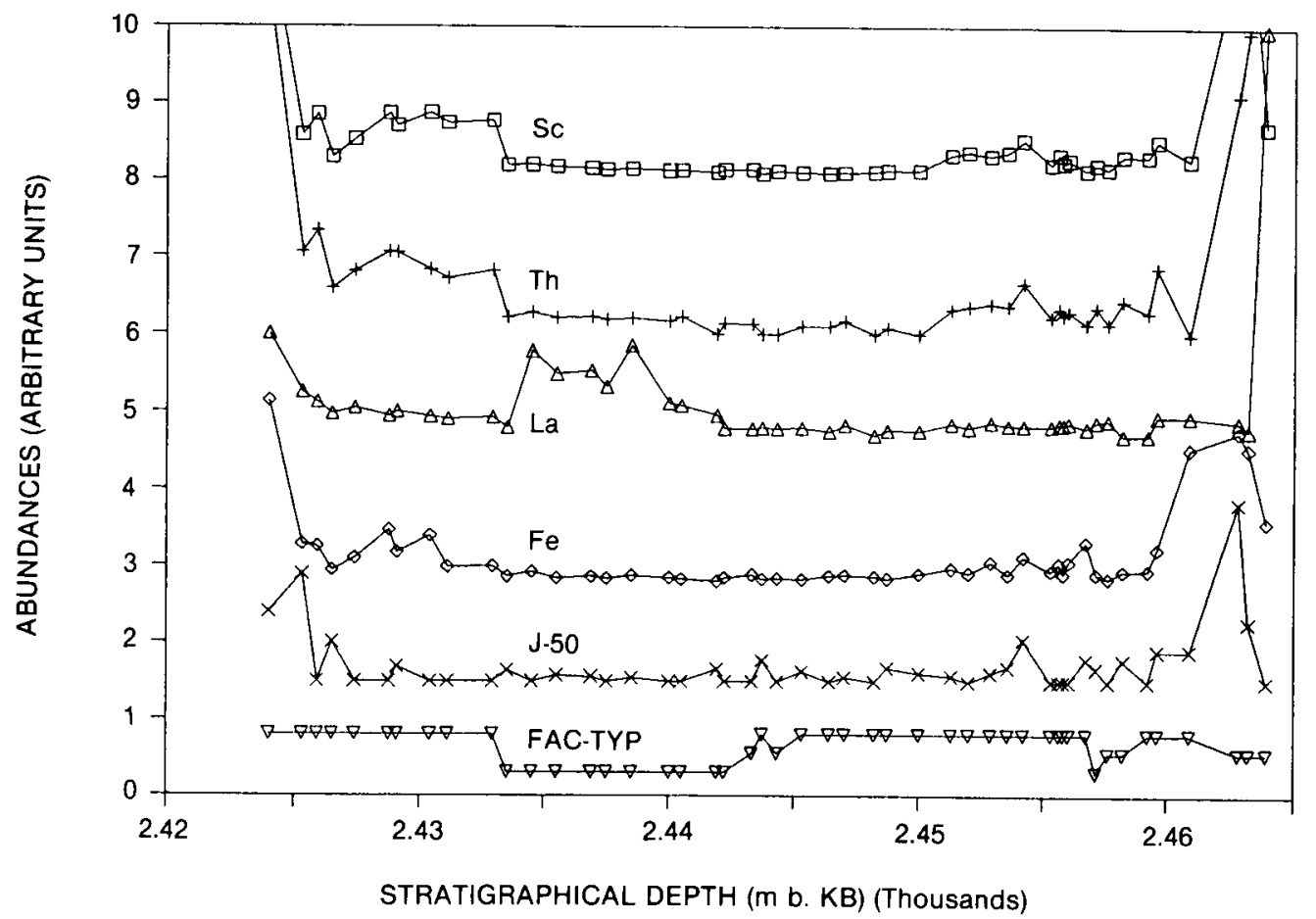

Fig. 25. Scandium, Th, Fe, La determined by INAA (Table 1), palaeomagnetic intensity (Table 5), and facies type versus depth. A positive correlation is observed between all these parameters, except with La in the top-most oolitic shoal facies interval. 


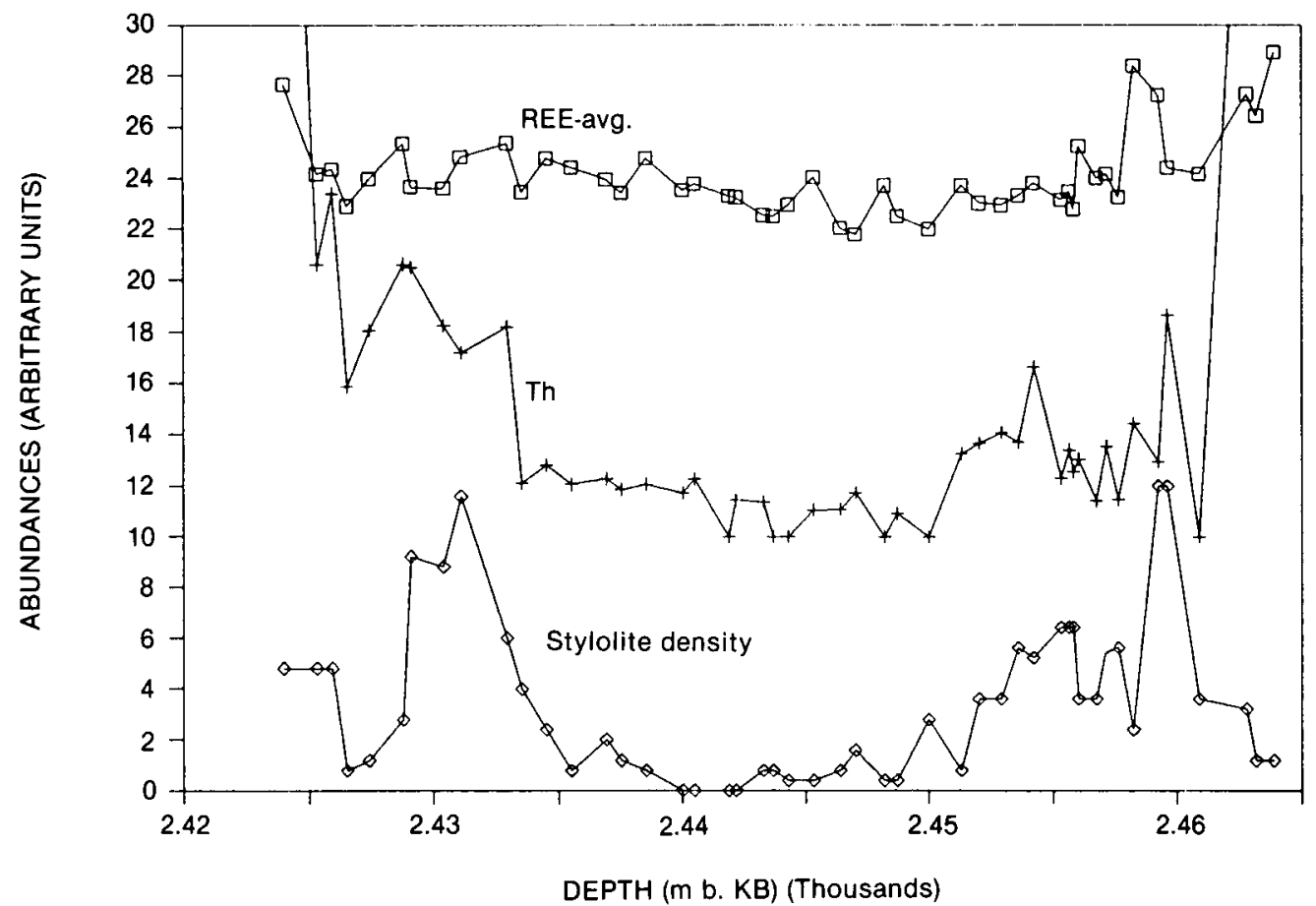

Fig. 26. Density of stylolites measured in number of stylolites per meter in the vertical direction, the total REE abundance (sum of all REE listed in Table 1), and Th abundance, as a function of stratigraphical depth.

muscovite and other clay minerals. The amount of REE present in the dolomite and anhydrite is thus considered small compared to the amounts bound to muscovite, clay minerals, and other related terrigenic minerals.

If the REEs are bound to muscovite, clay minerals, or other non-carbonate material it is not unreasonable to assume that there is a positive correlation between the pressuresolution responses (e.g. the stylolites) of the $\mathrm{Ca}-2$ rock and the concentrations of $\mathrm{REE}$, because pressure-solution concentrates the residuals, i.e. the chemically inert particles such as siliclastic sand, silt, and clay, as films on the stylolite surfaces. In the Ca-2 rock two types of pressure-solutions are seen (Figure 4), cf. Wanless (1979): (1) A sutured-seam solution where the response to overburden (stress) is solution along distinct stylolitic surfaces that undulate or form in- terpenetrating pillars and sockets with maximum extensional amplitudes of ca. $1 \mathrm{~mm}$. (2) A non-sutured seam solution of dark clayish(?) seams. These seams appear partly as a dense anastomosing network forming distinct bands of up to several $\mathrm{cm}$ thickness, partly as wispy flaser structures. The individual seams are a few $\mu \mathrm{m}$ in thickness. According to Wanless (1979) non-sutured solution surfaces may form in carbonate rock areas that contain more fine platy insoluble material (clay and platy silt) than the rock areas where common stylolites are formed. The density of stylolites (number of stylolites per meter in the vertical direction), the total REE abundance, and the Th abundance is shown as a function of stratigraphical depth on Figure 26. A fair correlation between stylolite seam density and REE abundance is seen on Figure 26, especially in the central parts of the $\mathrm{Ca}-2$ in- 
terval. This indicates that the REEs are in fact in some way bound to the insoluble remains (e.g. muscovite or clay minerals) of the $\mathrm{Ca}-2$ rock. The correlation between the lithophile element $\mathrm{Th}$ and the density of stylolites/solution seams is also very good. It should be noted, however, that the very pronounced rise in REE and Th abundances at the very top of the Ca- 2 interval does not correspond to a similar rise in the density of stylolites/solution seams, so at the extreme upper end of the interval the concentration of clays and other non-carbonate minerals must be of earlier (pre-pressure-solution) origin. The fact that the total REE abundance does not correlate as well with stylolite density as Th does, is partly due to an overrepresentation of light REE's in the total REE abundance, which is calculated as a simple sum of all REE abundances.

Besides this, a correlation between several REE, most pronounced for $\mathrm{La}$, is ob- served with facies type, which is shown on Figure 25 (see also Table 8). The two facies types are clearly distinguishable on e.g. a La versus Th plot (Figure 27).

The correlation between $\mathrm{La}$ and $\mathrm{Th}$ in sedimentary rocks has been discussed extensively in the literature. McLennan et al. (1980) found La/Th-ratios between 2 and 4 for various fine-grained rocks, and argued that this could be used to estimate the Th content of the upper crust. Parekh et al. (1977) found a linear correlation between bulk-sample La content and acid-insoluble residue. The Th content reported by Parekh et al. (1977) very closely reflects the content of acid-insoluble residue.

The fact that not quite as strong correlations is observed with $\mathrm{Th}$ and the other REE can be attributed to the fact that $\mathrm{La}$ is the most electropositive element of the REE, and that it therefore outwins the others in a competition for the superficial sites.

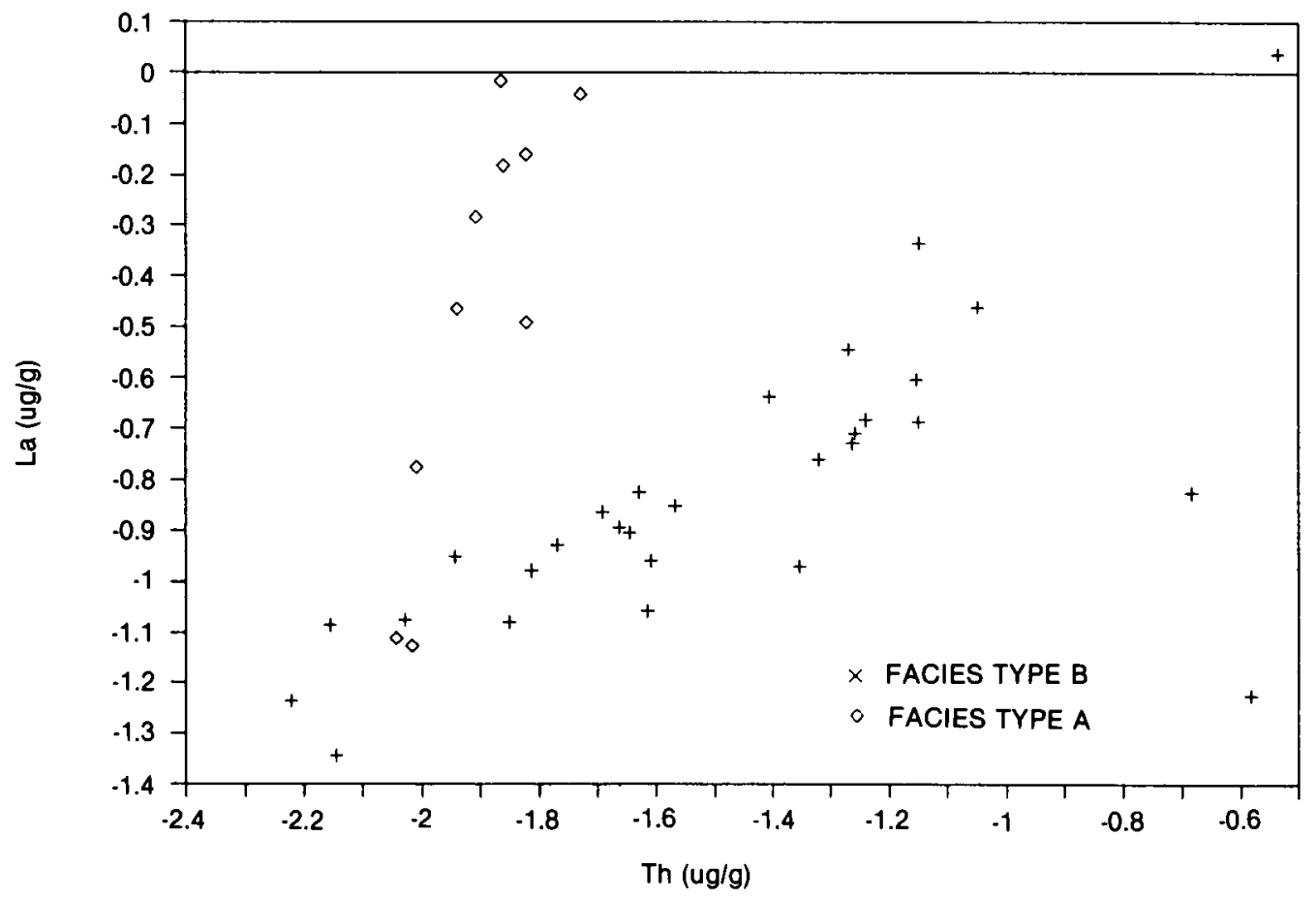

Fig. 27. $\log ($ La) determined by INAA (Table 1) versus $\log ($ Th) determined by INAA (Table 1). A positive linear correlation is observed for each facies. 
The combined data suggest that $\mathrm{Th}$ was originally in the clay minerals, whereas most of the REE were added to the surfaces of the clay mineral grains at a later date. It seems that precipitation of the REE onto the clay minerals directly on the ocean bottom can be excluded, because this would be expected to yield a large positive $\mathrm{Ce}$-anomaly, which is not observed. Likewise is an enrichment of clay minerals by pressure dissolution, giving rise to the abundant stylolites, an insufficient mechanism, because this process will not be able to account for the relationship between $\mathrm{La}$ and $\mathrm{Th}$. Neither is there any signs of gradients in the REE concentrations as argued for by e.g. Nelson (1987). It is remarkable that even though the Ca-2 interval has been subjected to several diagenetic events and thus abundant possibility of further migration of the REE (as argued for by McLennan \& Taylor, 1979) and the creation of concentration gradients, no such gradients are observed. Our data seem more in accordance with the mechanisms argued for by Ronov et al. (1967), where REE are shown to be mobilized in acidic stages and precipitated onto available surfaces in alkaline stages. In view of this it seems unlikely that for instance percolating seawater should have been responsible for the deposition of REE on the surface of in situ clay mineral grains, and we favor a scenario where the clay minerals receive their coating of REE prior to deposition on the Zechstein ocean bottom.

\section{Iron}

The occurrence of Fe has been thoroughly investigated in the present work. Following the AC-demagnetization measuring sequence, the samples were subjected to a DC-field of ca. 1890 Gauss in five minutes, which brought the magnetic minerals to saturation. The saturation magnetization rather closely follows the NRM as can be seen on Figure 6b. A few deviations do occur, however, mostly in the lower part of the interval, which could indicate that chemical process may have altered the magnetic minerals (or precipitated new minerals) in this part of the interval $(2453-2465$ $\mathrm{m} b$. KB). The susceptibility has also been measured (Table 5). The susceptibility is negative through the whole interval in concordance with the fact that the bulk of the material is anhydrite, dolomite or halite. Occasionally, however, the values varies indicating the presence of varying amounts of paramagnetic or ferromagnetic minerals in small amounts. It is seen on Figure $6 \mathrm{~b}$ that the susceptibility co-varies with the NRM and the saturation magnetization in the upper part of the interval, whereas it varies quite the opposite way (out of phase) in the lower part of the interval $(2453-2461 \mathrm{~m}$ b. $\mathrm{KB})$. Again this is an indication of different minerals carrying the magnetic signal in the upper and lower part of the Ca-2 interval. of the Ca-2 interval.

Concerning the rather high abundances of $\mathrm{Fe}$ in combination with relative high abundances of the trace elements $\mathrm{As}, \mathrm{Sb}$ and $\mathrm{Se}$ a sulphate phase might be expected. According to Onishi (1969) As may be present in iron sulfides in marine shales. Occurrence of the chalcophile element Se has been reported in a series of sulfide minerals (Leutwein, 1972). However, small amounts of Se may also be present in anhydrite. Badalov et al. (1969) studied the Se content of anhydrite and coexisting sulfides (pyrite, chalcopyrite and enargite), and found a Se content in anhydrite ranging from 0.8 to $3 \mu \mathrm{g} / \mathrm{g}$, while the Se content of the coexisting sulfides ranged from 30 to $220 \mu \mathrm{g} / \mathrm{g}$. Pyrite has been observed in the thin sections (Stentoft, 1990), but it seems not to be the major Fe bearing phase, because of the rather close correlation between $\mathrm{Fe}$ and palaeomagnetic intensity (Figure 25 and Table 5). This refusal of pyrite as a major Fe bearing phase is further substantiated by the fact that Fe correlates rather well with the dominantly lithophile element $\mathrm{Sc}$, as can be seen on Figure 18. The strong correlation between $\mathrm{Fe}$ and $\mathrm{Sc}$ indicates that the major $\mathrm{Fe}$ bear- 


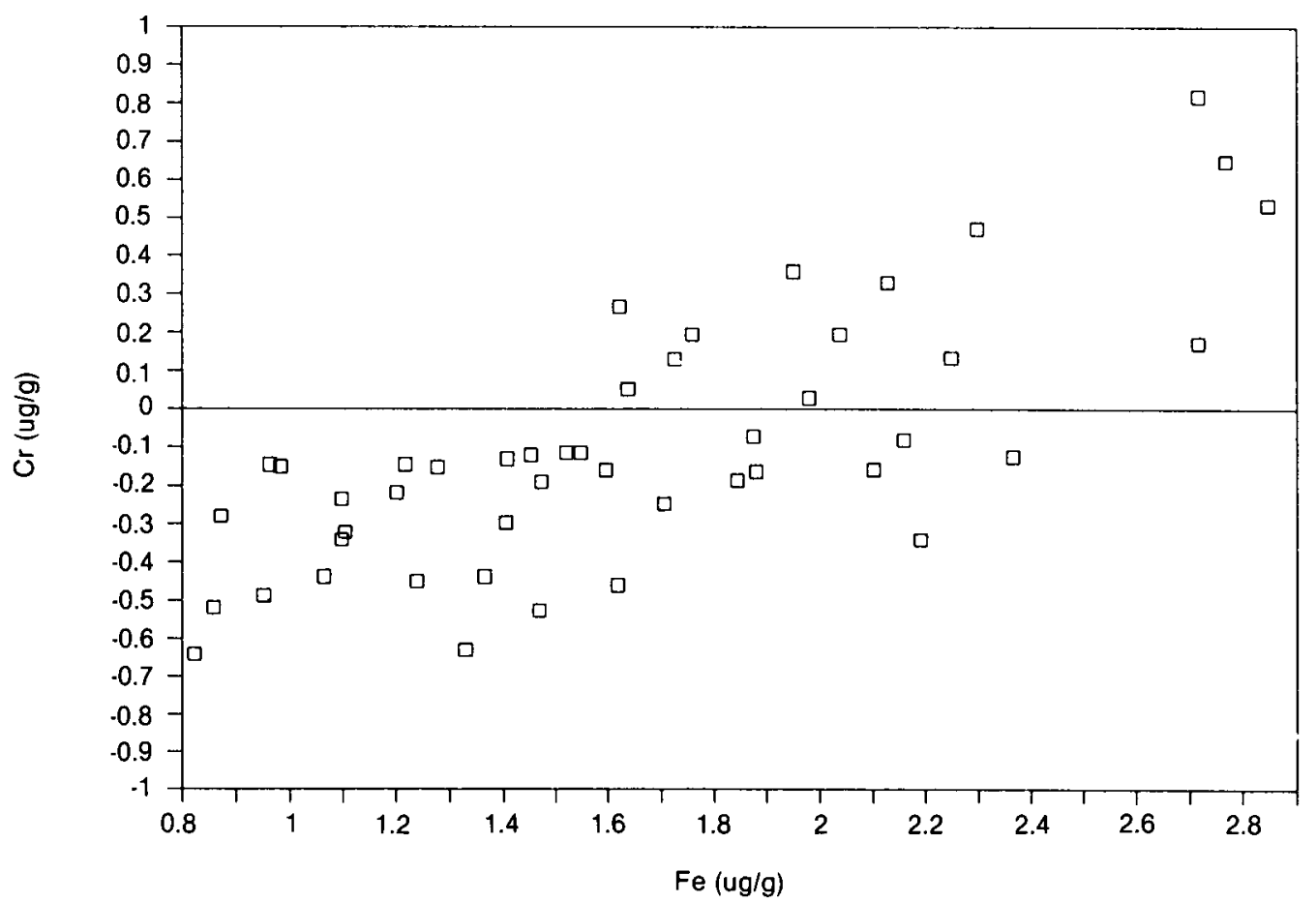

Fig. 28. $\log (\mathrm{Cr})$ determined by INAA (Table 1) versus $\log (\mathrm{Fe})$ determined by INAA (Table 1). A rather weak correlation is observed.

ing phase is a silicate mineral, or possibly although less likely a spinel. The best candidate for the rather moderate occurrence of Fe (ca. $100 \mu \mathrm{g} / \mathrm{g}$ on average) is the ubiquitous clay minerals.

The palaeomagnetic inclination as plotted in Figure 6c rather clearly exhibits a geomagnetically reversed interval between 2437 and $2447 \mathrm{~m} \mathrm{~b}$. KB. Several reversals are reported to have occurred in Zechstein times (Khramov et al., 1974, 1982; Dachroth, 1976; Vaughan \& Turner, 1980; Menning et al., 1988; Turner et al., 1989). As we have investigated only a very limited depth interval for palaeomagnetism and as the absolute dating of the $\mathrm{Ca}-2$ interval is relatively uncertain, we cannot draw firm conclusions as to precisely which geochronological reversal we have encountered. It remains, though, that one geomagnetically reversed period is present in the middle of the Ca-2 interval, and we speculate that it is identical to one of the five reversed periods reported in Thuringian (upper Tatarian) times by Dachroth (1976) and Menning et al. (1988).

\section{Antimony, As, and Se}

Antimony, As and Se are found in small concentrations in maltenes, asphaltenes, and bitumens from Alberta oil sands ( $\mathrm{Ja}$ cobs et al., 1984). Jacobs et al. (1984) found an As contents in organic compounds ranging from $0.06-0.5 \mu \mathrm{g} / \mathrm{g}, \mathrm{Sb}$ ranging from $0.006-0.17 \mu \mathrm{g} / \mathrm{g}$, and Se ranging from 0.01 $-1 \mu \mathrm{g} / \mathrm{g}$. Filby (1975) found $0.66 \mu \mathrm{g} / \mathrm{g}$ As in California crude oil and $2.25 \mu \mathrm{g} / \mathrm{g}$ in asphaltenes, and $0.52 \mu \mathrm{g} / \mathrm{g} \mathrm{Sb}$ in crude oil and 1.2 $\mu \mathrm{g} / \mathrm{g} \mathrm{Sb}$ in asphaltenes. Hitchon et al. (1975), on the other hand, found an average of $0.006 \mu \mathrm{g} / \mathrm{g}$ of $\mathrm{Sb}$ in Alberta crude oil, a factor of hundred lower than for the Cali- 
fornia oil. Concerning Se Filby (1975) found an average of $0.36 \mu \mathrm{g} / \mathrm{g}$, whereas Hitchon $e t$ al. (1975) found $0.05 \mu \mathrm{g} / \mathrm{g}$.

Even though some oil have been introduced in the Ca-2 interval, it seems unlikely that organic compounds can account fully for the amount of neither As (ca. $0.4 \mu \mathrm{g} / \mathrm{g}$ ), $\mathrm{Sb}$ (ca. $0.05 \mu \mathrm{g} / \mathrm{g}$ ), nor Se (ca. $0.2 \mu \mathrm{g} / \mathrm{g}$ ) found in the present study. It thus seems probable that the occurrence of $\mathrm{Sb}, \mathrm{As}$ and Se may be accounted for by the small amount of pyrite or other sulfide minerals present, and it is considered less likely, although not impossible, that $\mathrm{Sb}$, As and $\mathrm{Se}$ are either adsorbed to the clay minerals (Geering et al., 1968; Onishi, 1969) or partly originate from the heavy part of the crude.

\section{Chromium and $U$}

Chromium is known to occur adsorbed to clay minerals (Krauskopf, 1956), and ac- cording to several authors $\mathrm{Cr}$ in carbonate rocks correlates positively with their clay content (e.g. Parekh et al., 1977; Shiraki, 1978). Chromium is also prone to occur in heavy minerals, in which $\mathrm{Cr}$ may either substitute for $\mathrm{Fe}$ (e.g. in magnetite) or $\mathrm{Al}$ (in pyroxenes and amphiboles) in the octahedral sites of the minerals (Shiraki, 1978). Chromium is also reported in rather high abundances in asphaltenes, Jacobs et al. (1984) found up to $4.3 \mu \mathrm{g} / \mathrm{g}$ and Filby (1975) an average of $7.5 \mu \mathrm{g} / \mathrm{g}$. Asphaltenes constitutes only a small fraction of the crude and Filby (1975) found a Cr content of $0.6 \mu \mathrm{g} / \mathrm{g}$ in total California crude, whereas Hitchon et al. (1975) found an average of $0.09 \mu \mathrm{g} / \mathrm{g}$ in Canadian crude oil.

In the present study we find rather low $\mathrm{Cr}$ abundances (an average of $0.9 \mu \mathrm{g} / \mathrm{g}$ ). We consider it most likely that $\mathrm{Cr}$ primarily is present in scarce heavy mineral grains. The rather weak positive correlation with $\mathrm{Fe}$ (Figure 28) can thus be explained by the

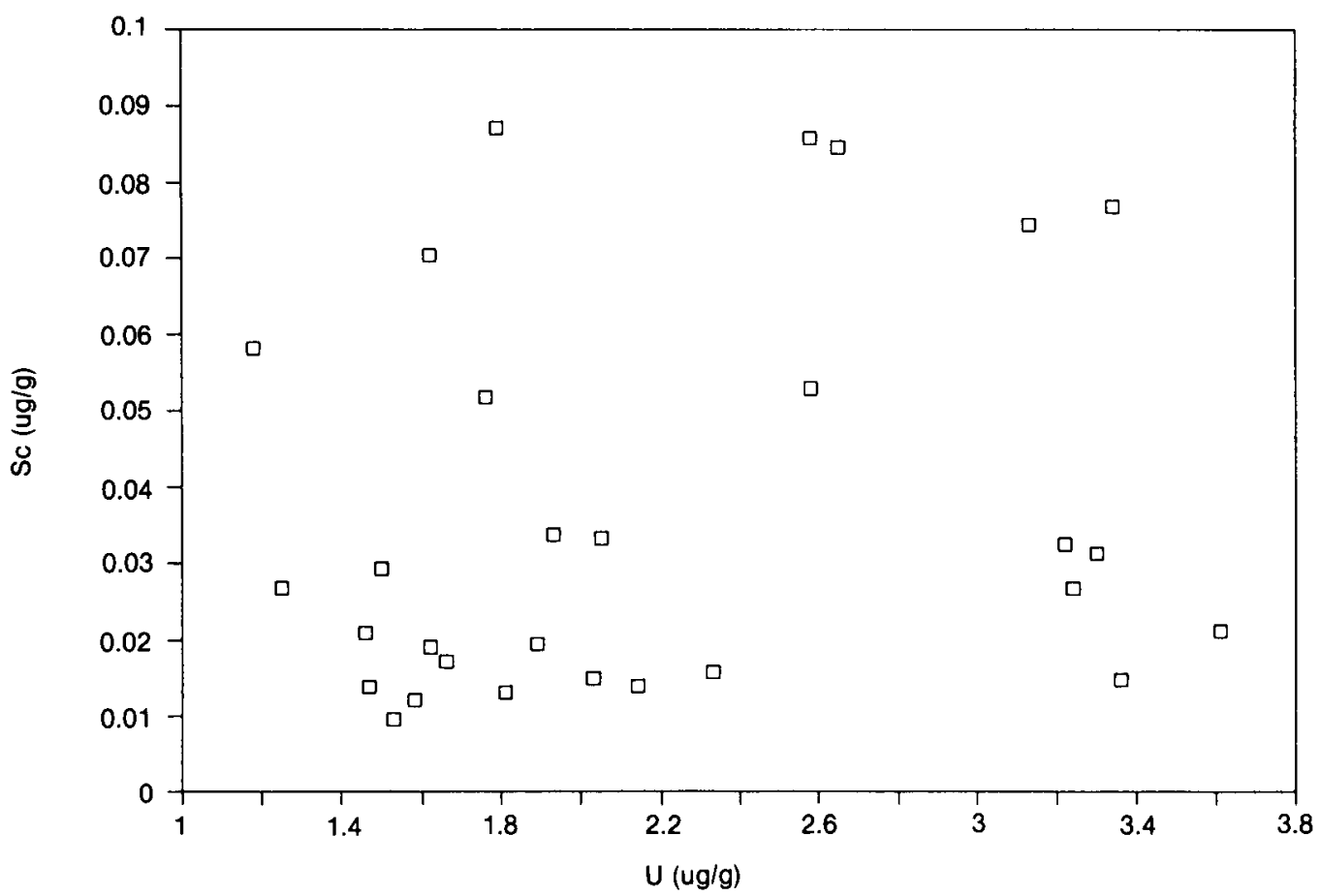

Fig. 29. Uranium determined by INAA (Table 1) versus Sc determined by INAA (Table 1). No significant correlation is observed. 


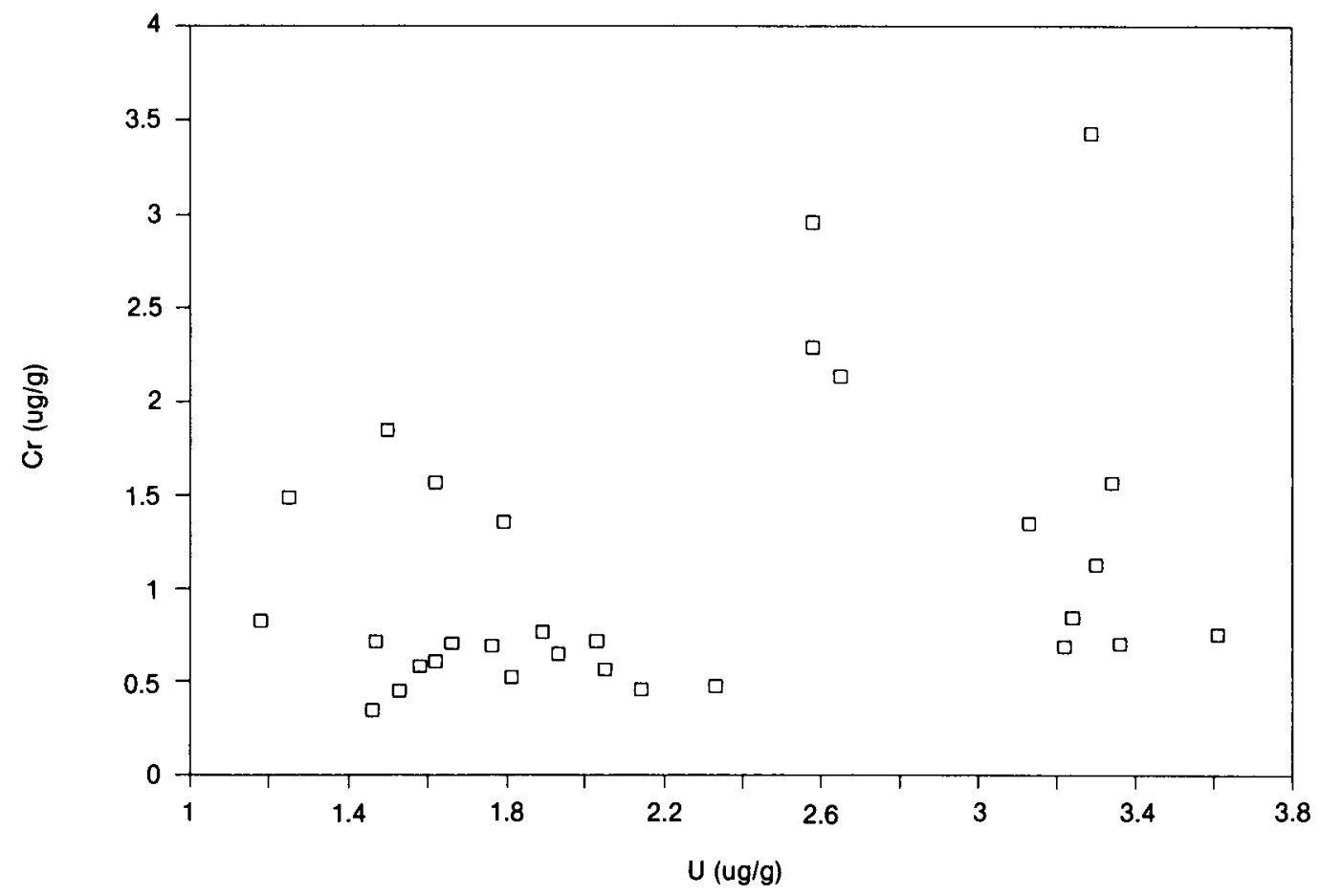

Fig. 30. Uranium determined by INAA (Table 1) versus Cr determined by INAA (Table 1). A weak correlation is observed.

bulk of the Fe being attached to the ubiquitous clays minerals. An organic origin of some of the $\mathrm{Cr}$ cannot, however, be completely be ruled out.

Uranium occurs in rather high abundances $(2.6 \mu \mathrm{g} / \mathrm{g}$ on average $)$. Uranium does not, as might have been expected, correlate with the other lithophile elements such as $\mathrm{Th}, \mathrm{Sc}$, or Fe (Figure 29). Uranium shows a weak correlation with $\mathrm{Cr}$ (Figure 30), which we interpret as an affinity for the heavy minerals. An interesting fact is that U correlates negatively with the content of anhydrite (Figure 31), and positively with the amount of dolomite. This could indicate that $U$ was present in the dolomite phase, maybe adsorbed to dark organic matter or forming organo-metallic compounds (Rogers \& Adams, 1969), and was later mobilized and removed during the diagenetic anhydritization process. Small amounts of the $\mathrm{U}$ may still be connected to whatever re- mains of dark organic matter in the dolomite phase.

It is with some hesitation that we ascribe $\mathrm{Cr}$ and $\mathrm{U}$ to a heavy mineral phase, because we did not identify such a phase by X-ray diffraction. A small heavy mineral phase is, however, very common in chalk (e.g. Hansen et al., 1988), and we consider it likely that the amount of heavy minerals present was too small for us to observe in our X-ray diffraction studies in comparison with the clay minerals and the quartz and opal.

\section{Hafnium, Ta, and Th}

It is conceivable that $\mathrm{Hf}$, which has been detected in $65 \%$ of the samples with a mean abundance of ca. $0.03 \mu \mathrm{g} / \mathrm{g}$, is adsorbed onto clay minerals. Experiments have shown that Hf, like $\mathrm{Zr}$, also occurs adsorbed onto $\mathrm{Mn}$ dioxide precipitated from seawater (Erlank 


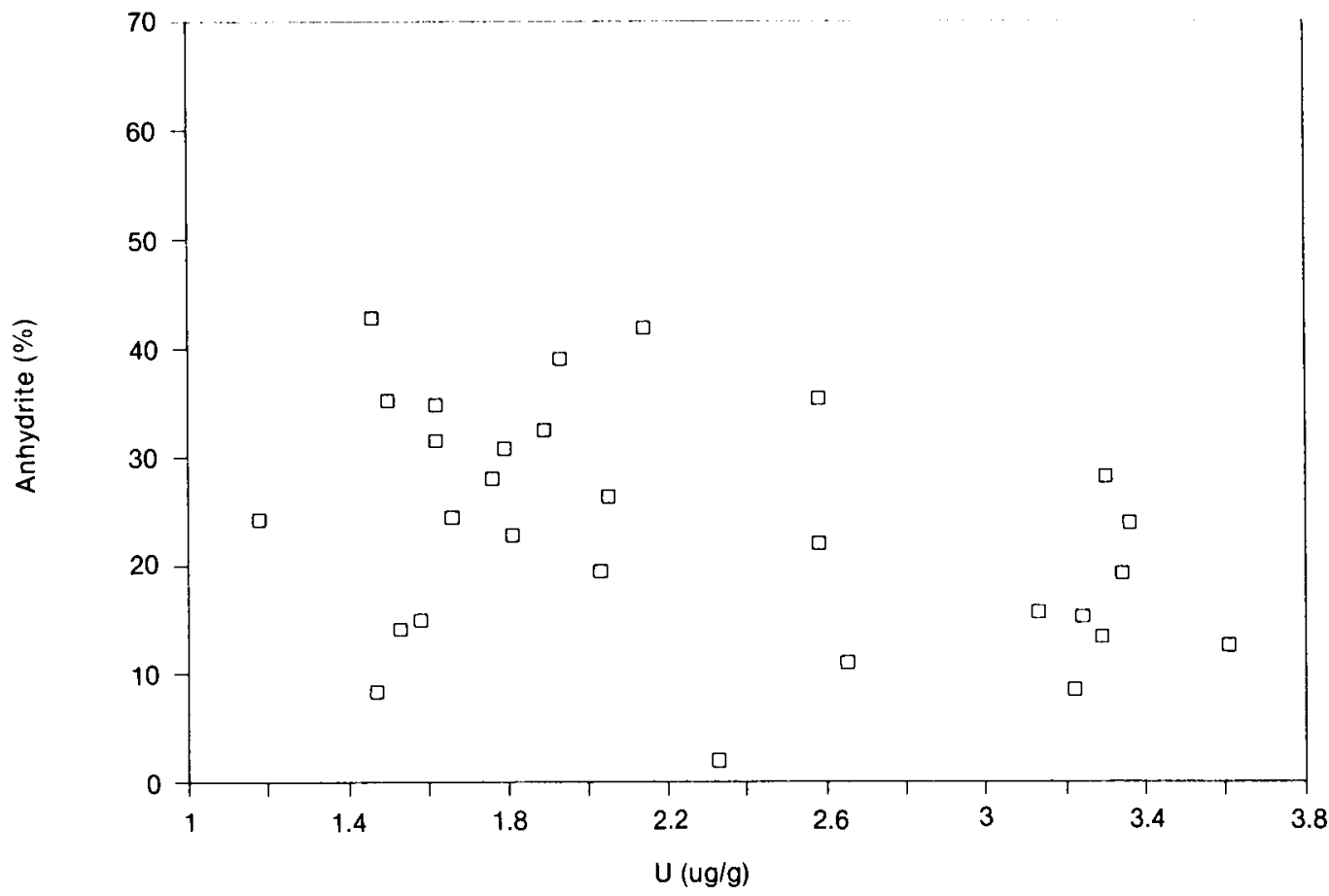

Fig. 31. Anhydrite content determined by atomic absorption (Table 2) versus $U$ determined by INAA (Table 1). A negative correlation is observed.

et al., 1978), but since such minerals are not encountered in the present rocks, it is considered an unlikely source for the Hf in the Ca-2 samples. Hafnium may also be present in detrital $\mathrm{Zr}$-minerals (e.g. zircon), oxides (e.g. ilmenite) and silicates (e.g. pyroxenes) or it may occur as hydrolyzates (Erlank et al., 1978). The hydrolyzate nature of Hf, Ta and $\mathrm{Th}$ is also demonstrated by Ronov \& Migdisov (1965). If Hf occurs in zircon grains it seems to be more mobile than $\mathrm{Zr}$, and thus more prone to enter the water phase (Portnov, 1965). We consider it likely that most of the $\mathrm{Hf}$ is of hydrolyzate nature.

Tantalum concentrations in the $\mathrm{Ca}-2$ samples are very low (ca. $0.02 \mu \mathrm{g} / \mathrm{g}$ on average) and above detection limit in only 4 samples all in the lagoonal carbonates facies type. We have found no reports on the occurrence of Ta, but it is widely known that V is ubiquitous in organic material. Vanadium has been detected in many crudes and residues
(Filby, 1975; Hitchon et al., 1975; Reynolds et al., 1984; Jacobs et al., 1984) so it might be suspected that Ta could be present there also. It does, however, seem more likely that Ta either occurs in heavy minerals, e.g. zircon, or that it occurs as hydrolyzates in clay minerals as do Hf and Th (Ronov \& Migdisov, 1965). In favor of the zircon origin speaks that Ta seems to prefer the heavy mineral assemblages, at least relative to $\mathrm{Nb}$ (Pachadzhanov, 1963). We consider the hydrolyzate origin the most likely.

Thorium most likely occupy interior sites in the clay minerals, as was argued above. This is in good agreement with a hydrolyzate origin (Ronov \& Migdisov, 1965). It is also in good agreement with a rather strong Th-Hf correlation as can be seen on Figure 32. The strong correlation between $\mathrm{Th}$ and Hf further substantiates the interpretation that $\mathrm{Hf}$ is also of hydrolyzate origin. 


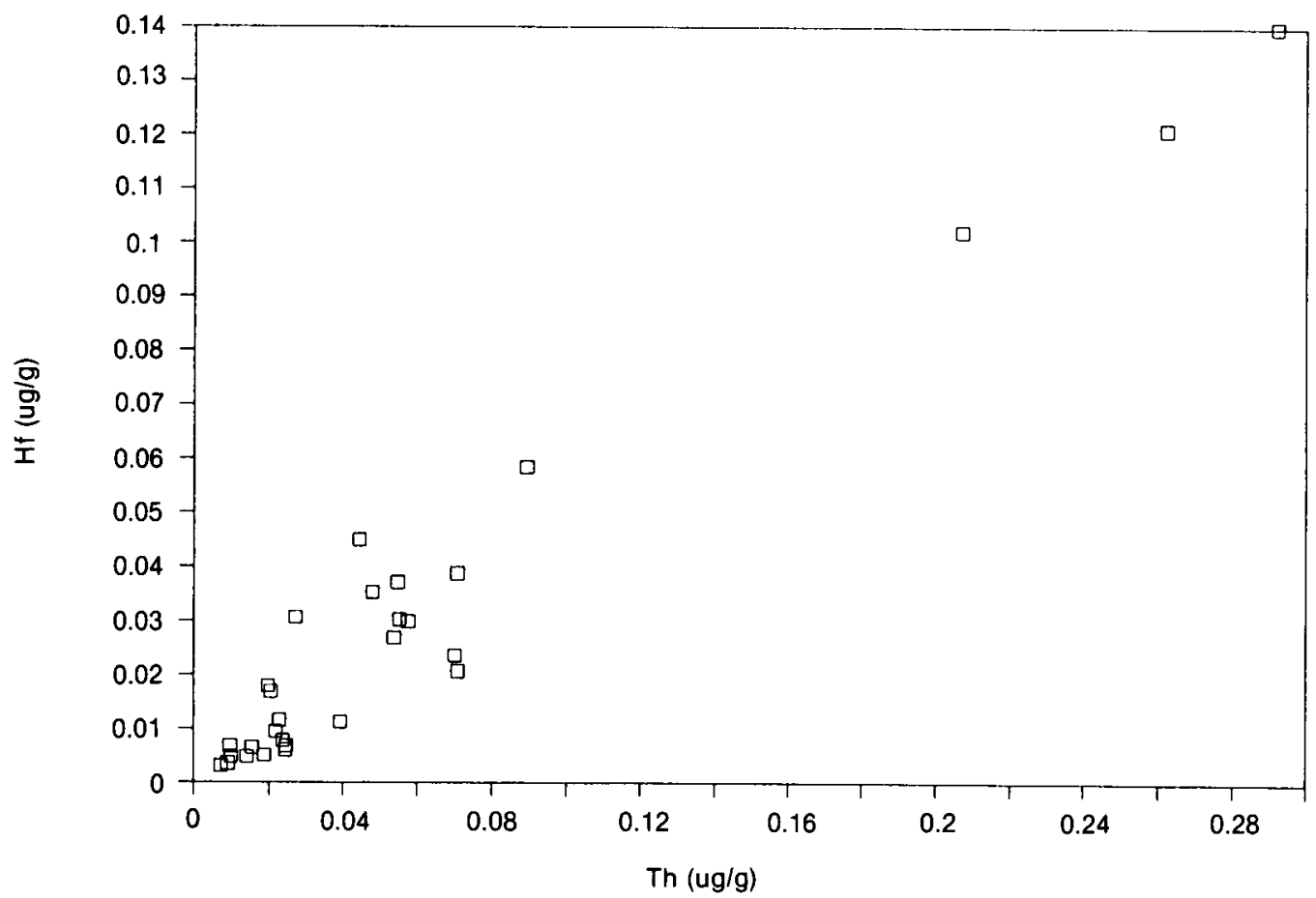

Fig. 32. Hafnium content determined by INAA (Table 1) versus Th determined by INAA (Table 1). $A$ rather strong correlation is observed.

\section{The highly siderophile elements}

Iridium have been determined in samples of EDTA-insoluble residue (the main results of which will be reported elsewhere, Stentoft et al., 1990). Most likely the Ir content originate from the infall of cosmic material on Earth (Alvarez et al., 1980; Grün et al., 1985; Maurette et al., 1987). This gives the possibility of determining the sedimentation rate of the sediment, provided that we knew the annual cosmic influx in Permian times. This is not known with any degree of certainty, but if we crudely assume that the influx is the same as the present day influx, ca. 20,000 tons CI material per year over the entire globe, we can convert the Ir abundance to sedimentation rate. The average $\mathrm{Ir}$ content of $0.006 \pm 0.001 \mathrm{ng} / \mathrm{g}$ corresponds to a sedimentation rate of 1 meter per $\mathrm{My}$, where we have used a CI Ir abundance of 480 ng/g (Wasson, 1985). It must be stressed, however, that this sedimentation rate is at best very approximative.

In the Ca-2 samples of Løgumkloster-1 the concentrations of $\mathrm{Hg}$ are quite high (average ca. $900 \mathrm{ng} / \mathrm{g}$ ) compared to average abundances of $\mathrm{Hg}$ in various types of sedimentary rocks, Turekian \& Wedepohl (1961) reports the following average $\mathrm{Hg}$ abundances: shale $400 \mathrm{ng} / \mathrm{g}$, sandstone 30 $\mathrm{ng} / \mathrm{g}$, carbonate rocks $40 \mathrm{ng} / \mathrm{g}$. The most likely reason for this, is contamination from $\mathrm{Hg}$-vapor from the laboratory. This interpretation is substantiated by a fair positive correlation between $\mathrm{Hg}$ and permeability (Figure 33). However, it is also possible, if not very likely, that $\mathrm{Hg}$ does originate from the dolomite. 


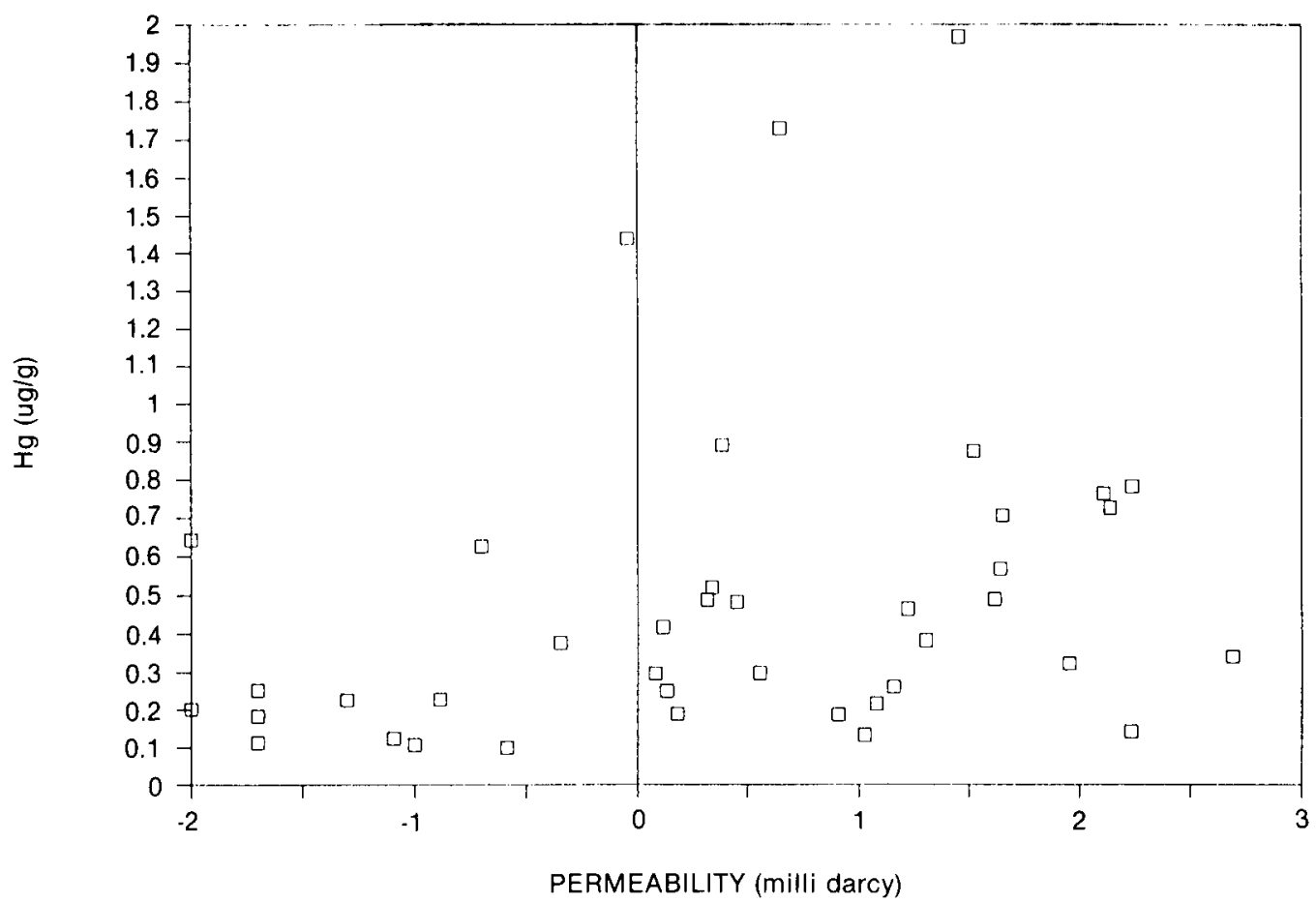

Fig. 33. Mercury determined by INAA (Table 1) versus logarithm of permeability (Table 6). A positive correlation is observed. It is thought that $\mathrm{Hg}$ is a contaminant, derived from Hg-fumes in the laboratory.

\section{The drilling mud}

Even though the rock samples for INAA have been taken from the central parts of the cores some contamination by the drilling mud might be suspected in the more permeable samples. In order to clarify this point we have analyzed four mud-samples from two levels ( $2435 \mathrm{~m}$ and $2455 \mathrm{~m}$ ) by INAA (Table 1). The mud-samples were taken both from the surface of the core section and from inside fractures formed by the relief of the pressure during the coring.

The drilling mud is a complex mixture of seawater, added substances (see Table 3) and dissolved or fragmented parts of the pierced rocks.

$\mathrm{X}$-ray diffraction of the drilling mud featured four distinct clay minerals, drastically different from the EDTA-insoluble residues of the rock samples.
The chemistry of one mud-sample turned out to deviate distinctly from the rest and was therefore excluded. The mean listed in Table 1 is the mean of the remaining three mud-samples. In Figure 34 is shown the ratio of the average mud composition to the average Ca- 2 composition (also listed in Table 1). It is worthwhile to note the following elements:

Calcium: The concentration of $\mathrm{Ca}$ is only half of that found in the rock samples, or ca. $12 \mathrm{wt} \%$. The larger part of this $\mathrm{Ca}$ is undoubtedly derived from the pierced carbonate rock (for instance the $\mathrm{Ca}-2$ dolomite itself) but some $\mathrm{Ca}$ has also been added (Table 3).

$\mathrm{REE}, \mathrm{Fe}, \mathrm{Sc}, \mathrm{As}, \mathrm{Sb}, \mathrm{Hf}, \mathrm{Th}, \mathrm{K}, \mathrm{Rb}$, and Cs: The Rare Earth Elements determined, $\mathrm{La}, \mathrm{Ce}, \mathrm{Nd}, \mathrm{Sm}, \mathrm{Eu}, \mathrm{Tb}, \mathrm{Yb}$, and $\mathrm{Lu}$ are probably for the most part adsorbed to the clay-minerals of the added Wyoming Bento- 


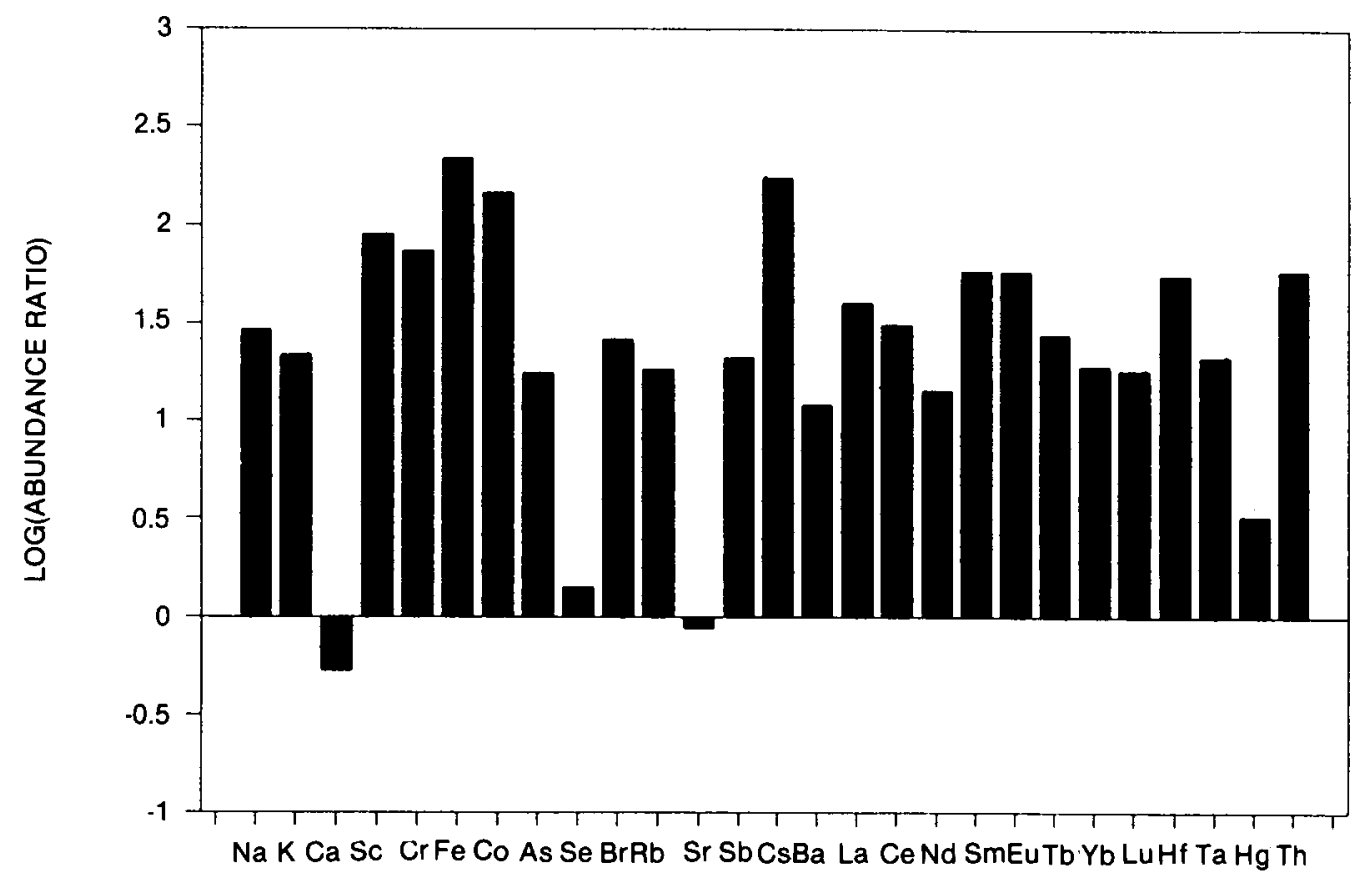

Fig. 34. Average mud composition determined by INAA (Table 1) normalized to average Ca-2 composition (Table 1).

nite (a montmorillonite) and the pierced clay intervals above the $\mathrm{Ca}-2$ carbonates. The high abundances of REE in the drilling mud as compared to the relatively clay poor Ca-2 rocks (by factors from ca. 3 to 58 ) are in good agreement with this origin. The fact that the REE contents in the Ca-2 samples to some degree is connected to the depositional environmental facies (as is discussed above) seems to rule out that the REE contents are primarily derived from contamination from the drilling mud. $\mathrm{Fe}, \mathrm{Sc}, \mathrm{As}$, $\mathrm{Sb}, \mathrm{Hf}, \mathrm{Th}, \mathrm{K}, \mathrm{Rb}$, and $\mathrm{Cs}$ are also rather heavily enriched in the drilling mud relative to the rock samples. This is considered in agreement with the hypothesis that these elements are primarily associated with the clay minerals, of which bentonite is mixed into the mud. It is interesting to note that $\mathrm{Fe}$ behaves differently from $\mathrm{Cr}$ and $\mathrm{Co}$, the latter two not being enriched to any significant degree in the drilling mud. This, however, is probably more diagnostic for the composition of the bentonite than for the formation.

Strontium, $\mathrm{Cr}, \mathrm{Co}, \mathrm{Se}$, and Ta: The $\mathrm{Sr}$ concentration in the drilling mud (ca. 2400 $\mu \mathrm{g} / \mathrm{g}$ ) is comparable to the concentrations in the dolomite samples. Strontium can substitute for $\mathrm{Ca}$ in dolomite and anhydrite (see discussion above). As there is no obvious source of $\mathrm{Sr}$ in the added substances, we believe that the $\mathrm{Sr}$ in the mud is derived from the pierced anhydrite and dolomite intervals above $\mathrm{Ca}-2$ (A-2, $\mathrm{Ca}-3$, etc.). Apart from $\mathrm{Sr}$ and $\mathrm{Ca}$, the only elements that seems to stem from disintegration of the formation carbonate are $\mathrm{Cr}, \mathrm{Co}, \mathrm{Se}$, and Ta.

Barium: Barium, which can also substitute for $\mathrm{Ca}$ in calcite, dolomite and anhydrite, is relatively enriched in the drilling mud compared with the rock samples (ca. $490 \mu \mathrm{g} / \mathrm{g}$ in the mud, and ca. $40 \mu \mathrm{g} / \mathrm{g}$ in the rock). The reason for this is probably that some barite $\left(\mathrm{BaSO}_{4}\right)$ has been added to the 
drilling mud, even if the operators mud report does not state this (Larsen, 1988).

Sodium and $\mathrm{Br}$ : The large abundances of these elements are probably primarily due to dissolution of the overlying $\mathrm{Z} 2$ salts $(\mathrm{Na}-2$ unit), and secondarily to the addition of halite (Table 3a). The concentrations of these elements in seawater is far less than encountered in the mud (Krauskopf, 1967, App. III).

Mercury: The concentration of $\mathrm{Hg}$ in one of the drilling mud samples is ca. 3 times higher than in the formation rocks. This is an interesting, if not alarming, fact. The reason for this enhancement is unknown to us, but we speculate that the added cellulose (Drispac, in Table 3) was stained with $\mathrm{Hg}$. The $\mathrm{Hg}$ is definitely not contamination from $\mathrm{Hg}$-vapor in the laboratory, which we think could have been the case for the rock samples, because the mud samples were never stored in our laboratory. In light of these findings it is considered wise to monitor the content of $\mathrm{Hg}$ in drilling mud during future drill operations, as it could constitute an environmental hazard as well as a health hazard to the people working on the rig. 


\section{Conclusions}

The geochemical characterization of the $\mathrm{Ca}-2$ rock samples and samples of the drilling mud has led to the following results:

1) It seems that the drilling mud did not contaminate the core in any of the elements investigated in this study, with the possible exception of $\mathrm{Hg}$.

2) Strontium and $\mathrm{Ba}$ is found to reflect the original (prediagenetic) conditions of the environment. Both $\mathrm{Sr}$ and $\mathrm{Ba}$ reach high values almost exclusively in the lagoonal carbonate facies. The diagenetically derived anhydrite is quite low in $\mathrm{Sr}$, but a subgroup of 27 samples with increasing amounts of anhydrite show a slight increase in its (low) Sr content. The CInormalized REE-pattern show a small but distinct negative Ce-anomaly for the oolitic shoal facies subgroup, whereas no such Ce-anomaly is seen for the lagoonal carbonates facies subgroup. This is in accordance with the oolitic shoal facies being in close and high energy contact with seawater, and the lagoonal facies being completely or periodically isolated from seawater.

3) Sodium and $\mathrm{Br}$ form a co-varying group distinct from the group consisting of $\mathrm{K}$, $\mathrm{Rb}$, and $\mathrm{Cs}$. The bulk of the $\mathrm{Na}$ and $\mathrm{Br}$ is interpreted to be situated in halite, which has been observed in small quantities throughout the unit.

4) Iron, Sc, Th, Co, K, Rb, Cs and the bulk of the REE are interpreted to be situated in the small EDTA-insoluble fraction, which has been identified to consist primarily of muscovite and other clay minerals.

5) Hafnium, Ta, and Th are interpreted as being of hydrolyzate origin, and thus now being incorporated into the bulk of the muscovite and clay minerals.

6) $\mathrm{Cr}$ and $\mathrm{U}$ do not correlate or correlates poorly with the rest of the lithophile elements. We interpret these elements to be situated in a heavy mineral phase (spinels) of very small volumetric abundance. Some of the U may still be connected to minute amounts of dark organic matter left in the rock.

7) Antimony, As, and Se are interpreted to be situated in a mass-wise small pyrite fraction. An organic origin, from the heavy part of the crude, cannot completely be ruled out.

8) The rather high amounts of $\mathrm{Hg}$ present in the rock samples (ca. $0.9 \mu \mathrm{g} / \mathrm{g}$ ) are thought to be caused by contamination from $\mathrm{Hg}$ fumes in the laboratory. This is substantiated by positive correlation between $\mathrm{Hg}$ and permeability.

9) A geomagnetic reversal is encountered in the middle of the Ca-2 interval, and it is tentatively identified as one of the Tatarian reversal events.

The results of the present work accentuate the necessity of coring large sections in full core during drill operations. 


\section{Acknowledgements}

Niels Oluf Jørgensen and Henning Christiansen provided helpful and constructive reviews. Raymond Gwozdz, Christian Koch and Simon Andersen are thanked for discussions. We thank Raymond Gwozdz of TraceChem for performing the INAA analysis and Christian Kock for performing the XRD analysis. Hans Jørgen Hansen and the Geologic Central Institute at University of
Copenhagen is thanked for the use of the electron microscope. Troels Laier is thanked for performing the atomic absorption analysis. The Danish Natural Science Research Council supplied the electron microscope, the SQUID-magnetometer, and the KLY-2 Kappabridge susceptibility-meter. This work was supported by EEC-grant contract No. EN3C/003-DK(MB). 


\section{References}

Abbey, S., 1983: Studies in "Standard Samples" of silicate rocks and minerals 1969-1982. Geological Survey of Canada, paper 83-15.

Alvarez, L. W., Alvarez, W., Asaro, F., \& Michel, H. V., 1980: Extraterrestrial cause for the Cretaceous-Tertiary extinction. Science, 208, 10951108.

Baartman J. C., 1978: In Rasmussen, L. B. (ed.): Geological aspects of the Danish North Sea sector. Danmarks Geologiske Undersøgelse, Serie III, no. 44,85 pp.

Badalov, S. T., Belopolskaya, T. L., Prikhidko, P. L., \& Turesebekov, A., 1969: Geochemistry of selenium in sulfate-sulfide mineral parageneses. Geochemistry International, 6, 799-802.

Banner, J. L., Hanson, G. N., \& Meyers, W. J., 1988: Rare earth element and $\mathrm{Nd}$ isotopic variations in regionally extensive dolomites from the Burlington-Keokuk formation (Mississippian). Implications for REE mobility during carbonate diagenesis. Journal of Sedimentary Petrology, 58, 415-432.

Bathurst, R. G. C., 1976: Carbonate sediments and their diagenesis. Developments in Sedimentology, 12, Elsevier Scientific Publishing Company, Amsterdam, $658 \mathrm{pp}$.

Botz, R. \& Müller, G., 1981: Mineralogie, Petrographie, anorganische Geochemie und IsotopenGeochemie der Karbonatgesteine der Zechstein 2. Geologisches Jahrbuch, Reihe D, D47, 3-112.

Carr, M. \& Turekian, K. K., 1961: The geochemistry of cobalt. Geochimica et Cosmochimica Acta, 23, 9-60.

Chester, R., 1965: Adsorption of zink and cobalt on illite in sea-water. Nature, 206, 884-886.

Clark, D. N., \& Tallbacka, L., 1980: The Zechstein deposits of Southern Denmark. Contributions to Sedimentology, 9, 205-231.

Dachroth, W., 1976: Gesteinsmagnetische Marken im Perm Mitteleuropas (Ein Beitrag zur Anwendung gesteinmagnetischer Daten in der Stratigraphie). Geologisches Jahrbuch, Reihe E, Heft $10,3-63$.

Dansk Boreselskab, 1981: Løgumkloster-1 Completion Report, June 1981 (Internal report).

Deer, W. A., Howie, R. A., \& Zussman, J., 1976: An introduction to the rock-forming minerals. Longman. $528 \mathrm{pp}$.
Dunham, R. J., 1962: Classification of carbonate rocks according to depositional texture. Memoires of the American Association of Geology, 1, 108121.

Erlank, A. J., Smith, H. S., Marchant, J. W., Cardoso, M. P., \& Ahrens, L. H., 1978: Hafnium. In: K. H. Wedepohl (ed.): Handbook of Geochemistry, vol. Il/5. Springer Verlag, Berlin.

Felsche, J., 1978: Yttrium and Lanthanides. In: K. H. Wedepohl (ed.): Handbook of Geochemistry, vol. II/5. Springer Verlag, Berlin.

Filby, R. H., 1975: The nature of metals in petroleum. In: T. F. Yen (ed.): The role of trace metals in petroleum, 31-59. Ann Arbor Science Publishers, Ann Arbor.

Fischer, K., 1972: Barium. In: K. H. Wedepohl (ed.): Handbook of Geochemistry, Chapter 56A. Springer Verlag, Berlin.

Fleet, A. J., 1984: Aqueous and Sedimentary Geochemistry of the Rare Earth Elements. In: P. Henderson (ed.): Rare Earth Element Geochemistry, p. 343-373

Flügel, E., 1978: Mikrofazielle Untersuchungsmethoden von Kalken. Springer Verlag, Berlin, 454 pp.

Friedman, G. M., 1969: Trace elements as possible environmental indicators in carbonate sediments. In: G. M. Friedman (ed.): Depositional environments in carbonate rocks. SEPM Special Publications, no. 14, 193-198.

Fuge, R., 1974: Bromine. In: K. H. Wedepohl (ed.): Handbook of Geochemistry, vol. II/3. Springer Verlag, Berlin.

Geering, H. R., Cary, E. E., Jones, L. H. P., \& Allaway, W. H., 1968: Solubility and redox criteria for the possible forms of selenium in soils. Soil Society of America, Proceedings, 32, 35-40.

Gendron, C. R., Cahill, R. A., \& Gilkeson, R. H., 1988: Comparison of spectral gamma ray (SGR) well logging data with instrumental neutron activation analysis (INAA) data for rock types in Northern Illinois. The Log Analyst, 29, 345-357.

Goldschmidt, V. M., 1954: Geochemistry. Oxford University Press, Oxford, $730 \mathrm{pp}$.

Goni, J. \& Guillemin, C., 1964: Sites of trace elements in minerals and rocks. Geochemistry International, 1, 1025-1034.

Govindaraju, K., 1984: Geostandards Newsletter, 8, Special Issue, July 1984. 
Graf, J. I.., 1984: Fffects of Mississippi Valley-type mineralization on REE patterns of carbonate rocks and minerals, Viburnum Trend, Southeast Missouri. Journal of Geology, 92, 307-324.

Grün, E., Zook, H. A., Fechtig, H., \& Giesse, R. H., 1985: Collisional balance of the meteoritic complex. Icarus, 62, 244-272.

Hansen, H. J., Gwozdz, R., \& Rasmussen, K. L., 1988: High-resolution trace element chemistry across the Cretaceous-Tertiary boundary in Denmark. Revista Espanola de Paleontologia, No. Extraord., 21-29.

Haskin, L. A., Haskin, M. A., Frey, F. A., \& Wildeman, T. R., 1968: Relative and absolute terrestrial abundances of the rare earths. In: L. H. Ahrens (ed.): Origin and Distribution of the Elements. Pergamon, Oxford, p. 889-912.

Haynes, B. W., Law, S. L., Barron, D. C., Kramer, G. W., Maeda, R., \& Magyar, M. J., 1985: Pacific Manganese Nodules: Characterization and Processing. Bulletin 679, U.S. Department of the Interior, Bureau of Mines, $44 \mathrm{pp}$.

Heier, K. S. \& Billings, G. K., 1970: Cesium. In: K. H. Wedepohl (ed.): Handbook of Geochemistry, vol. II/4. Springer Verlag, Berlin.

Hitchon, B., Filby, R. H., \& Shah, K. R., 1975: Geochemistry of trace elements in crude oils, Alberta, Canada. In: T. F. Yen (ed.): The role of trace metals in petroleum, 31-59. Ann Arbor Science Publishers, Ann Arbor.

Jacobs, F. S., Bachelor, F. W., \& Filby, R. H., 1984: Trace metals in Alberta oil sand bitumen components. In Characterization of heavy crude oils and petroleum residues (Symposium, Lyon 25-27 June 1984). Technip, Paris. 173-178.

Jarvis, J. C., Wildeman, T. R., \& Banks, N. G., 1975: Rare earths in the Leadville limestone and its marble derivatives. Chemical Geology, 16, 27-37.

Khramov et al., 1974: Ref. in Menning et al. (1988).

Khramov et al., 1982: Ref. in Menning et al. (1988).

Kocman, V. \& Woodger, S. C., 1986: Certificate of analysis: Four North-American gypsum rock samples type: Calcium sulfate dihydrate. Domtar Inc. Senneville, Quebec, Canada H9X 3 L7.

Krauskopf, K. B., 1956: Factors controlling the concentrations of thirteen rare metals in sea-water. Geochimica and Cosmochimica Acta, 9, 1-32B.

Krauskopf, K. B., 1967: Introduction to Geochemistry. McGraw-Hill, Tokyo. 617 pp.

Kushnir, J., 1980: The coprecipitation of strontium, magnesium, sodium, potassium and chloride ions with gypsum. An experimental study. Geochimica et Cosmochimica Acta, 44, 1471-1482.

Larsen, J., 1988: Mærsk Oil and Gas A/S. Personal communication.

Leutwein, F., 1972: Selenium. In: K. H. Wedepohl (ed.): Handbook of Geochemistry, vol. II/3. Springer Verlag, Berlin.
I.ogan, B. W., Rerak, R., \& Ginshurg, R. N., 1964 : Classification and environmental significance of algal stromatolites. Journal of Geology, 72, 68-83.

Macpherson, G. L., Farr, M. R., \& Posey, H. H., 1988: Rare earth element concentrations in formation waters, Gulf Coast sedimentary basin. SEPM Annual Midway Meeting (abstract), 5, p. 33.

Mason, B., 1966: Principles of Geochemistry. John Wiley, New York. 329 pp.

Maurette, M., Jéhanno, C., Robin, E., \& Hammer, C. U., 1987: Characteristics and mass distribution of extraterrestrial dust from the Greenland ice cap. Nature, 328, 699-702.

McLennan, S. M., Nance, W. B., \& Taylor, S. R., 1980: Rare earth element-thorium correlations in sedimentary rocks, and the composition of the continental crust. Geochimica et Cosmochimica Acta, 44, 1833-1839.

McLcnnan, S. M. \& Taylor, S. R., 1979: Rare earth element mobility associated with uranium mineralisation. Nature, 282, 247-250.

Menning, M., Katzung, G., \& Lützner, H., 1988: Magnetostratigraphic investigations in the Rotliegendes (300-252 Ma) of Central Europe. Zeitschrift für Geologische Wissenschafter, 16, 10451063 .

Nelson, K. L., 1987: Geochemical evaluation of diagenetic processes in a deep-water carbonate. Chemical Gcology, 64, 239-258.

Onishi, H., 1969: Arsenic. In: K. H. Wedepohl (ed.): Handbook of Geochemistry, vol. II/3. Springer Verlag, Berlin.

Oudin, E., \& Cocheric, A., 1988: Fish debris record the hydrothermal activity in the Atlantis II Deep sediments (Red Sea). Geochimica et Cosmochimica Acta, 52, 177-184.

Pachadzhanov, D. N., 1963: Geochemistry of niobium and tantalum in clays. Geochemistry, 963-976.

Palmer, M. R., 1985: Rare earth clements in foraminifera tests. Earth and Planetary Science Letters, 73, 285-298.

Parchk, P. P., Möller, P., Dulski, P., \& Bausch, W. M., 1977: Distribution of trace elements between carbonate and non-carbonate phases of limestone. Earth and Planetary Science Letters, 34, 39-50.

Phillipsborn, H. von, 1967: Tafeln zum Bestimmen der Minerale nach äusseren Kennzeichen. E. Schweizerbart'sche Verlagsbuchhandlung, Stuttgart. $319 \mathrm{pp}$

Pingitore, N. E., 1982: The role of diffusion during carbonate diagenesis. Journal of Sedimentary Petrology, 52, 27-39.

Portnov, A. M., 1965: The zirconium: hafnium ratio in minerals of the Burpala Massif. Geochemistry International, 2, 238-241.

Rao, C. P. \& Naqvi, I. H., 1977: Petrography, ge- 
ochemistry and factor analysis of a lower ordovician subsurface sequence, Tasmania, Australia. Journal of Sedimentary Petrology, 47, 1036-1055.

Reynolds, J. G., Briggs, W. R., Fetzer, J. C., Gallegos, E. J., Fish, R. H., Komlenic, J. J., \& Wines, B. K., 1984: Molecular characterization of vanadyl and nickel non-porphyrin compounds in heavy crude petroleums and residua. In Characterization of heavy crude oils and petroleum residues (Symposium, Lyon 25-27 June 1984). Technip, Paris. 153-157.

Richter-Bernburg, G., 1955: Stratigraphische Gliederung des deutschen Zechsteins. Zeitschrift deutscher Geologischer Gesellschaft, 105, 843-854.

Roaldset, E. \& Rosenqvist, I. T., 1971: Adsorbed rare earth elements as a clue to the origin of some glacial clays. Bulletin Groupe française Argiles, XXIII, 191-194.

Rogers, J. J. W. \& Adams, J. A. S., 1969: Thorium and Uranium. In: K. H. Wedepohl (ed.): Handbook of Geochemistry, vol. II/5. Springer Verlag, Berlin.

Ronov, A. B., Balaskov, Y. A., \& Migdisov, A. A., 1967: Geochemistry of the rare earths in the sedimentary cycle. Geochemistry International, 4, 1-17.

Ronov, A. B. \& Migdisov, A. A., 1965: Principal features of the geochemistry of hydrolyzate elements in weathering and sedimentation. Geochemistry International, 2, 92-117.

Saban, M., Vitorovic, O., \& Vitirovic, D., 1984: Correlation of crude oils from Vojvodina (Yugoslavia) based on trace elements. In Characterization of heavy crude oils and petroleum residues (Symposium, Lyon 25-27 June 1984). Technip, Paris. 122-127.

Shah, K. R., Filby, R. H., \& Haller, W. A., 1970: Determination of trace elements in petroleum by neutron activation analysis. Journal of Radioanalytical Chemistry, 6, 185-192.

Shaw, H. F., \& Wasserburg, G. J., 1985: Sm-Nd in marine carbonates and phosphates: Implications for Nd isotopes in seawater and crustal ages. Geochimica et Cosmochimica Acta, 49, 503-518.

Sicgel, F. R., 1979: Geochemical prospecting. Review of research on modern problems in geochemistry, 16, 273-290.

Shiraki, A. J., 1978: Chromium. In: K. H. Wedepohl (ed.): Handbook of Geochemistry, vol. II/3. Springer Verlag, Berlin.

Smykatz-Kloss, W., 1966: Sedimentpetrographische end Geochemische Untersuchungen an Karbonatgesteinen der Zechsteins, Teil I und II. Contr. Mineral. and Petrol., 13, 207-268.
Stentoft, N., 1990: Diagenesis of the Zechstein Ca-2 carbonate from the Løgumkloster 1 well, Denmark. Danmarks Geologiske Undersøgelse, Serie B, No. 12 .

Stentoft, N, \& Nygaard, E., 1985: On the sedimentology and diagenesis of the basal Zechstein carbonate unit in the Aabenraa-1 well, Denmark. Danmarks Geologiske Undersøgelse, Internal Report No. 11.

Stentoft, N., Frykman, P., Rasmussen, K. L., \& Koch, C. J. W., 1990 submitted: EDTA-insoluble residues from the $\mathrm{Ca}-2$ unit (Late Permian), the Løgumkloster-1 well, Denmark. Geologie en Mijnbouw.

Turekian, K. K., 1978: Cobalt. In: K. H. Wedepohl (ed.): Handbook of Geochemistry, vol. II/3. Springer Verlag, Berlin.

Turekian, K. K. \& Wedepohl, K. H., 1961: Distribution of the elements in some major units of the earth's crust. Bulletin of the Geological Society of America, 72, 175-191.

Turner, P., Turner, A., Ramos, A., \& Sopena, A., 1989: Paleomagnetism of Permo-Triassic rocks in the Iberian Cordillera, Spain: acquisition of secondary and characteristic remanence. Journal of the Geological Society, London, 146, 61-76.

Usdowski, E., 1973: Das geochemische Verhalten des Strontiums bei der Genese und Diagenese von Ca-Karbonat- und Ca-Sulfat-Mineralen. Contr. Mineral. and Petrol., 38, 177-195.

Vaughan, D. J., \& Turner, P., 1980: Diagenesis, magnetization and mineralization of the Marl Slate. Contr. Sedimentology 9, 73-90.

Veizer, J., 1978: Strontium. In: K. H. Wedepohl (ed.): Handbook of Geochemistry, vol. II/4. Springer Verlag, Berlin.

Veizer, J., 1983: Chemical diagenesis of carbonates: theory and application of trace element technique. In: M. A. Arthur et al. (eds): Stable Isotopes in Sedimentary Geology, SEPM Short Course No. 10, Chap. 3.

Veizer, J. \& Demovic, R., 1974: Strontium as a tool in facies analysis. Journal of Sedimentary Petrology, 44, 93-115.

Vine, J. D., Tourtelot, E. B., \& Elizabeth, B., 1970: Geochemistry of black shale deposits - a summary report. Economic Geology, 65, 253-272.

Wanless, H. R., 1979: Limestone response to stress: pressure solution and dolomitization. Journal of Sedimentary Petrology, 49, 437-462

Wasson, J. T., 1985: Meteorites - Their Record of Early Solar-System History. W. H. Freeman, New York. 267 pp. 
Rock samples of the Zechstein Ca-2 unit from the Løgumkloster-1 well have been characterized by neutron activation, atomic absorption, X-ray diffraction, palaeomagnetism, and optical and electron microscopical examination.

Imprints of both the original facies and the diagenetic events are found in the present work. Strontium and Ba abundances are thus reflecting the original conditions of the environment, which is also reflected in the normalized REE distribution patterns. Iron, Sc, REE and several other elements are found to be situated in the EDTA-insoluble residue, which has been identified primarily as a muscovite or clay mineral phase. A geomagnetically reversed period is encountered in the middle of the Ca-2 interval, and is tentatively identified as one of the Tatarian reversed events. 